Portland State University

PDXScholar

Fall 11-7-2016

\title{
A Community Engaged Approach to Address Intimate Partner Violence among Sexual Minority Women
}

Rachel Marie Smith

Portland State University

Follow this and additional works at: https://pdxscholar.library.pdx.edu/open_access_etds

Part of the Domestic and Intimate Partner Violence Commons, and the Lesbian, Gay, Bisexual, and Transgender Studies Commons

Let us know how access to this document benefits you.

\section{Recommended Citation}

Smith, Rachel Marie, "A Community Engaged Approach to Address Intimate Partner Violence among Sexual Minority Women" (2016). Dissertations and Theses. Paper 3323.

https://doi.org/10.15760/etd.3304

This Thesis is brought to you for free and open access. It has been accepted for inclusion in Dissertations and Theses by an authorized administrator of PDXScholar. Please contact us if we can make this document more accessible: pdxscholar@pdx.edu. 
A Community Engaged Approach to Address Intimate Partner Violence among Sexual Minority Women

\author{
by \\ Rachel Marie Smith
}

A thesis submitted in partial fulfillment of the requirements for the degree of

\author{
Master of Science \\ in \\ Psychology
}

Thesis Committee:

Eric Mankowski, Chair

David Morgan

Greg Townley

Portland State University

2016 


\section{(C) 2016 Rachel Marie Smith}

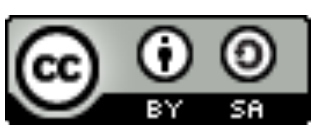

A Community Engaged Approach to Address Intimate Partner Violence among Sexual Minority Women by Rachel Marie Smith is licensed under a Creative Commons Attribution-ShareAlike 4.0 International License. 
Abstract

In response to a dearth of empirical literature concerning the mechanisms underlying female same-sex intimate partner violence (FSSIPV) perpetration, the purpose of this research is to inform intimate partner violence intervention and prevention strategies specific to sexual minority women. In particular, this research aims to inform a working intersectional model predicting FSSIPV perpetration, and to evaluate the face validity and construct coverage of existing survey measures related to gender, minority stress, and violence. Fourteen lesbian, gay, bisexual, transgender, and queer women recruited from the greater Portland, Oregon area participated in a series of in-depth, semistructured, open-ended one-on-one and focus group interviews. Participant recruitment involved a combination of purposive and convenience sampling methods aided by the involvement of multiple community partners working in violence and education related fields. Interview and focus group questions addressed participants' experiences with gender role stress and minority stress. Grounded theory analysis of participants' narrative responses informed the coverage and relevance of constructs in a working intersectional model predicting women's use of violence in their same-gender intimate relationships. In particular, findings indicate that sexual minority women's experiences of gender role stress and minority stress, particularly in combination, were especially influential on their identities. Sexual minority women's experiences with minority stressors were not confined to minority stressors specific to their gender identities and sexual orientations, but intersected with minority stressors related to race and class as well. These findings support an intersectional and contextually-minded approach to IPV intervention and prevention strategies. 
Dedication

This thesis is dedicated to the study's participants and the community partners who helped ensure the project's success: Bradley Angle; Men Engaging Now; the Northwest Network of Bisexual, Trans, Lesbian, and Gay Survivors of Abuse; the Oregon Attorney General's Sexual Assault Task Force; the Oregon Coalition against Domestic and Sexual Violence; Portland State University's Queer Resource Center; Portland State University's Women's Resource Center; Q-Center; the Tri-County Network of Batterer Intervention Providers; and the YWCA of Greater Portland. 


\section{Acknowledgements}

This project would not have been possible without the incredible support, guidance, and patience provided by my research advisor and thesis committee chair, Dr. Eric Mankowski. His unwavering encouragement of my pursuit of this work over the past three years combined with his expertise, thoughtfulness, and scientific curiosity not only played a critical role in the completion of this project, but also enhanced the overall quality of the project and the knowledge I have gained in completing it.

I would also like to thank Drs. David Morgan and Greg Townley for serving as members of my thesis committee, as well as for their guidance and support in my graduate training. Dr. Townley's expertise regarding participatory research methodologies with marginalized populations not only informed the overall design and analytic approaches in this project, but also challenged me to think beyond the scope of the project's specific target population characteristics. I am also grateful for Dr. Morgan's pragmatic approach to qualitative and mixed methods design. Throughout the course of data collection and analysis, I often found myself returning to my notes from the three courses I took with Dr. Morgan and to the wealth of methodological insights his work provides to anyone conducting qualitative and/or mixed methods research.

I am also immensely grateful to Nancy Greenman, who despite her busy schedule as the Prevention Program Coordinator for the Oregon Sexual Assault Task Force at the time, served as an invaluable consulting community partner throughout the course of this project. However, Nancy's role in this project began well before even the first draft of this project's proposal when she 'took me under her wing', so to speak, as an intern to the Oregon Sexual Assault Task Force's Prevention and Education Subcommittee. Her 
constant dedication and enthusiasm for this work is truly a force to be reckoned with, and has been a source of critical encouragement to me in doing this work.

This project's success thus far is also indebted to the support and guidance I received from Drs. Sarah Cook, Dominic Parrott, and Kevin Swartout at Georgia State University during my undergraduate training. Their support and instruction in my final two years at Georgia State University not only heavily influenced my successful application to Portland State University's Doctoral program, but also provided me with a unique level of theoretical and methodological insight that ultimately inspired this project.

Finally, I am infinitely grateful to my family's love, support, and patience during this process. I could not do any of this without them.

This material is based upon work supported by the National Science Foundation Graduate Research Fellowship. Any opinion, findings, and conclusions or recommendations expressed in this material are those of the authors(s) and do not necessarily reflect the views of the National Science Foundation. Fellow ID: 2014173965. 


\section{Table of Contents}

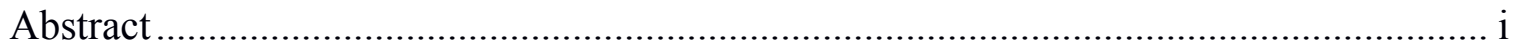

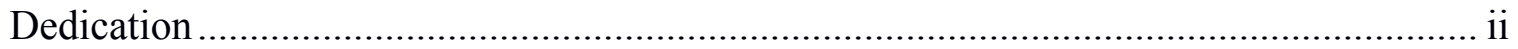

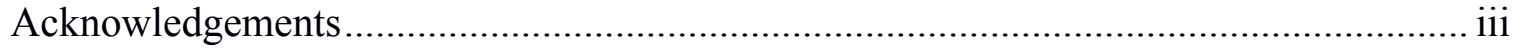

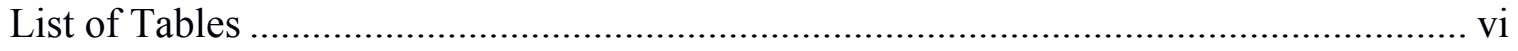

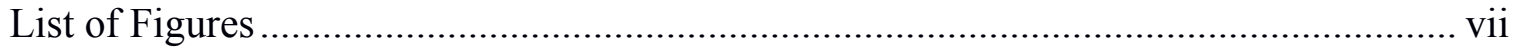

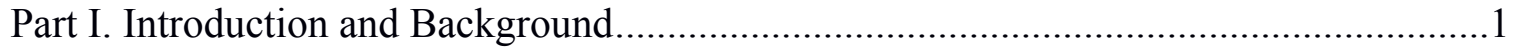

Chapter 1. Problem Statement ..................................................................................2

Chapter 2. Contextual Literature Review: Current and Historical Barriers to Examining Same-Gender IPV among Women .............................................. 12

Chapter 3. Conceptual Literature Review: Overview of a Working Intersectional Model Predicting Female Same-Sex IPV .........................................................35

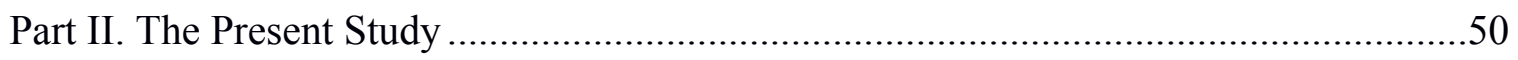

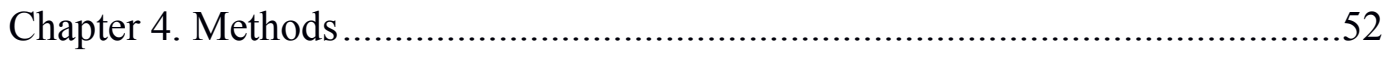

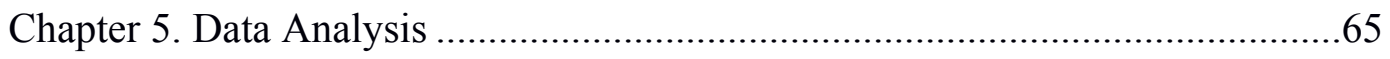

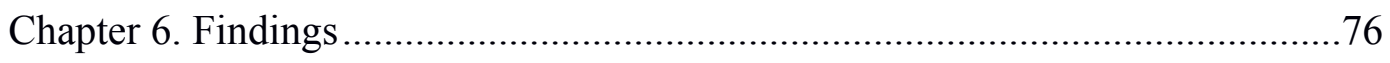

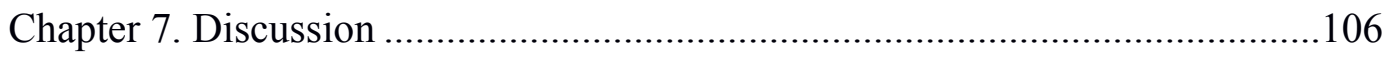

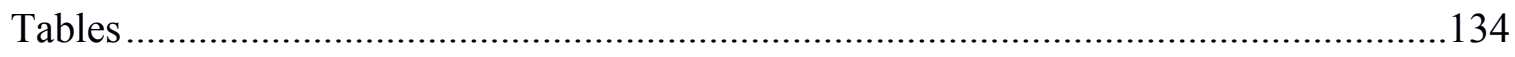

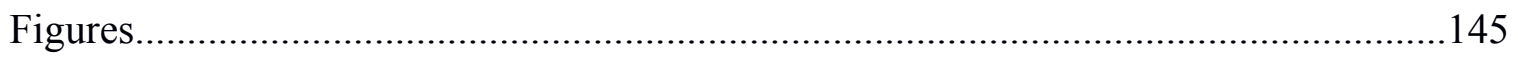

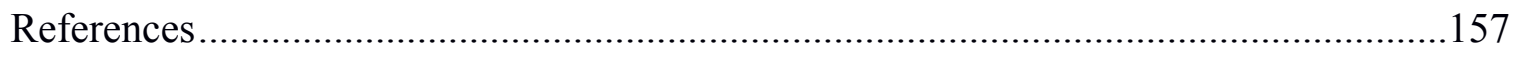

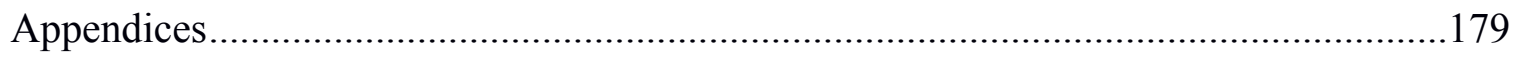

Appendix A. Focus Group Guide ............................................................ 180

Appendix B. One-on-One Interview Guide ................................................... 184

Appendix C. Inductively-Derived Codebook ................................................188 


\section{List of Tables}

1. Gender-Relevant and Violence Assessment Measures...........................................134

2. Participant Eligibility Criteria from Initial Focus Groups Design.............................135

3. Participant Screening Interview and Post-Interview/Post-Focus Group Survey

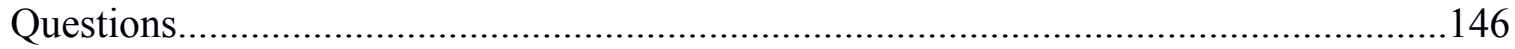

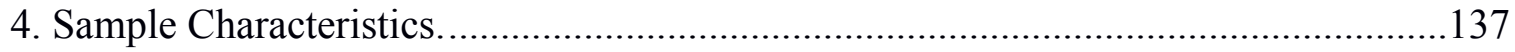

5. Sample Excerpt of Coded Interview Data Segment. ...............................................138

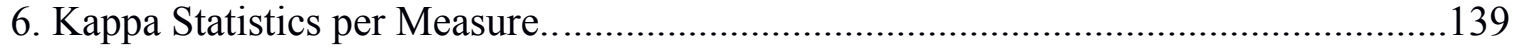

7. Codes with Disagreements Across All Measures. ....................................................140

8. Codebook Coverage per Measurement Category and per Measure............................142

9. Codes not Covered by Any of the Evaluated Measures. ..........................................143 


\section{List of Figures}

1. Matrix Visualization of Bem (1981b)'s multi-dimensional gender framework...........145

2. A Working Intersectional Model Predicting Female Same-Sex IPV (FSSIPV) .........146

3. R. M. Smith et al (2015)'s Mediation models of antifemininity, sexual dominance, and stress associated with subordination to women on sexual coercion.

4. Balsam \& Szymanski (2005)'s Mediational Model Predicting Female Same-Sex IPV

Perpetration and Victimization with Supportive Path Analysis Statistics......................148

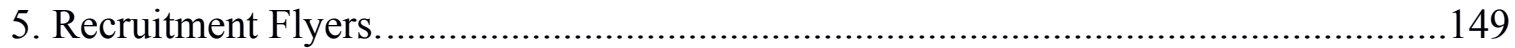

6. Timeline of Participation Dates and Recruitment Efforts......................................150

7. Reverse-Funnel Design (Morgan, 1996b) for Interview and Focus Group Guides.....151

8. Framework for evaluating overlap and disconnect among measurement tools and IPV-

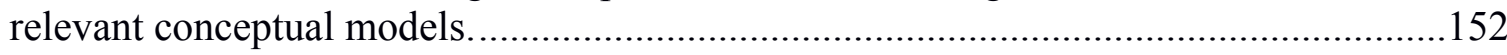

9. Combined Overlap among Codebook and Each Measurement Category. ..................153

10. Combined Overlap among Codebook and All Evaluated Measures. ........................154

11. I. H. Meyer (2003)'s Figure 1: "Minority Stress Processes in lesbian, gay, and

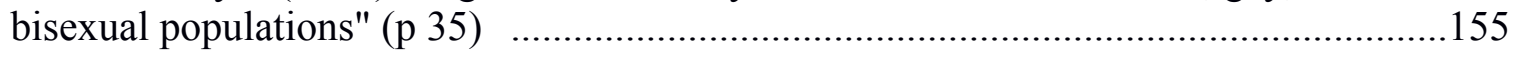

12. I. H. Meyer (2003)'s conceptual model of minority stress (MS; see I H Meyer, 2003, Figure 1) with proposed additions based on the amended minority stress construct definition. 


\section{Part I. Introduction and Background}

Before the reader is shunted through the relatively uncharted, often even hypothetical territory which lies before him, it is perhaps only fair he be equipped with some general notion of the terrain. (Millett, 2000, p. xix)

The following research report begins with a two-part description of the social problem motivating the present research: (1) the prevalence of intimate partner violence (IPV) among women in same-gender relationships and the continued lack of empirically comprehensive understandings of the mechanisms underlying this phenomenon's existence, and (2) the neglectful, stereotyping, and re-oppressive treatment of female same-sex intimate partner violence (IPV) by scientific research, and the communitybased services and policies based on such research, relevant to intimate partner violence. After providing these present contexts for the current study, I offer a historical analysis of the socio-political climates that have influenced the current state of the literature relevant to female same-sex intimate partner violence. Following a critical analysis of this history and of the resulting currently available modes of assessing, reporting, and understanding female same-sex IPV, I begin the second part of this report by presenting the specific constructs and mechanisms contained within a working intersectional model predicting women's use of violence in their same gender relationships, which served as a guiding theoretical model for the current study. I then provide the methodology and findings for this study, and the implications of this study's findings for refining the working intersectional model and future directions for the larger program of research in which the current project is situated. 


\section{Chapter 1. Problem Statement}

The dominant voice that has formed the epistemic understanding of violence against women has been most clearly articulated within a heterosexual paradigm. (Davis \& Glass, 2011, p. 13)

This study is ultimately focused on exploring the possible causes and correlates around sexual minority women's perpetration of abuse toward their same-gender partners in order to inform intervention and prevention strategies specific to same-gender intimate partner violence among women. Over the past several decades, there has been a considerable development of research and community action across the globe working to address intimate partner violence (IPV). In the United States, this research and activism stemmed primarily from first-and-second-wave feminist movements, which collectively led way to the U.S. Violence Against Women and, more specifically, the U.S. Domestic Violence Movement beginning around the 1980s. The public policies and communitybased services specific to intimate partner violence resulting from this mainstream work continue to be almost exclusively focused on men's abuse toward women (e.g., M. C. Black et al., 2011; Tjaden \& Thoennes, 2000a). Consequently, intimate partner violence occurring among members of the Lesbian, Gay, Bisexual, Transgender, and Queer (LGBTQ) population has been critically overlooked and the IPV-related needs of this population have gone unmet with severe consequences (Ristock, 2001; Walters, Chen, \& Breiding, 2013). Importantly, recent nation-level data indicate that LGBTQ individuals experience IPV at rates comparable to heterosexual individuals, and are in many ways at higher risk of experiencing violence than heterosexual population (Walters et al., 2013). Pertinent to this study, these data provide evidence that the prevalence of female same- 
sex intimate partner violence (FSSIPV) in particular occurs at much higher rates than expected by researchers conducting national-scale intimate partner violence survey work (Walters et al., 2013). This, along with historic data corroborating these statistics, suggests that the prevalence of FSSIPV has been grossly misrepresented throughout the U.S. Women's and Domestic Violence Movements. The causes and consequences of this misrepresentation lie in historical notions that regard female same-sex intimate partner violence as a phenomenon that is either non-existent (Cecere, 1986), not possible (Hammond, 1989), or not worthy of consideration based on anti-LGBTQ ideological viewpoints (Durish, 2011; Ristock, 2001, 2011). The relative lack of research on female same-sex IPV is characteristic of a long history of oppressions committed against sexual and gender minorities and traditional gender role stereotypic expectations of women to be nonviolent, or the notion that women do not possess the ability to be violent, or at least violent enough to inflict pain on others (Gilbert, 2002; Girshick, 2002; Hassouneh \& Glass, 2008; Little \& Terrance, 2010).

In line with the heteronormative stereotypic notion of women's incapability of violence, FSSIPV-inclusive research to date has tended to fall into one or both of the following categories: (1) reports of the estimated national prevalence of FSSIPV; and/or (2) heterosexual groups' and individuals' gender role stereotypes about women's samegender relationships, and the possibility of violence in those relationships (e.g., McClennen, 2005; Messinger, 2011). Compared to attention given to issues collectively faced by the LGBTQ population, the relatively large dedication of federal, state, and private funding for research related to violence against women and domestic violence since the original signing of the Violence Against Women Act (VAWA) has been almost 
exclusively used for developing services for presumed heterosexual, cis-gender, female survivors of abuse perpetrated by male intimate partners with similarly presumed heterosexual and cis-gender characteristics (Ristock, 2001). However, results of research related to IPV occurring within this narrowly defined population are not necessarily representative of the prevalence, causes, and correlates of IPV experienced by members of the LGBTQ population (Burke \& Follingstad, 1999; Hassouneh \& Glass, 2008; Ristock, 2001). Thus, domestic violence services that emerged out of the U.S. Women's and Domestic Violence movements of the 1980s and 1990s (e.g., emergency shelters for survivors and batterer intervention programs), which are largely based on this heterosexual-IPV-focused research, remain ill-equipped to serve LGBTQ IPV survivors, perpetrators, and their families (Hart, 1986; Lobel, 1986; McLaughlin \& Rozee, 2001; Ristock, 2001; C. Smith, 2011). In recent years, however, researchers have begun to address the lack of empirical knowledge around the prevalence and causes of intimate partner violence among Lesbian, Gay, Bisexual, Trans, and Queer populations by adding distinctions of sexual orientation to existing national intimate partner violence inquiries and reports (Walters et al., 2013).

\section{FSSIPV Prevalence and Rates}

Walters et al. (2013) recently augmented findings from the 2010 report of the National Intimate Partner and Sexual Violence Survey (NISVS, M. C. Black et al., 2011), the most up-to-date nationally representative intimate partner and sexual violence survey. In Walters et al. (2013)'s secondary report, the authors distinguished victimization and perpetration rates based on sexual orientation and found that $43 \%$ of lesbian women experience rape, stalking, and/or physical abuse by an intimate partner at least once in 
their lifetime, and that the prevalence of victimization among lesbian women is similar to, and slightly higher than, heterosexual women (35\%). These data further indicated that rates of abuse are significantly higher among bisexual women, relative to lesbian and heterosexual women, such that $61 \%$ of bisexual women reported victimization of intimate partner abuse. Among lesbian women who reported experiencing IPV victimization at least once in their lifetimes, approximately two-thirds reported only female perpetrators, indicating that female same-sex IPV is not a rarely occurring phenomenon simply explainable by individual characteristics of the perpetrators (i.e., the "bad apple" excuse; Ristock, 2001, 2003). Rather, these data provide support for the notion that FSSIPV is a social problem whose prevalence is comparable to that of heterosexual (male-to-female) IPV, and is influenced and maintained by ecological and systematic factors surrounding the seemingly individualist act of perpetrating violence toward an intimate partner (Ristock, 2001, 2003; Walters et al., 2013).

\section{Heterosexist Gender Role Stereotyping of Lesbian Romantic Relationships and FSSIPV}

Importantly, the findings presented by Walters et al. (2013) serve two purposes: (1) to estimate and report the extent of IPV among the U.S. lesbian, gay, and bisexual population (no distinctions were made regarding transgender individuals), and (2) to reiterate findings from previous inquiries exploring the prevalence of IPV among this population (McLaughlin \& Rozee, 2001; Morrow \& Hawxhurst, 1989; Renzetti, 1992, 1998; Ristock, 2001, e.g., 2002; Robson, 1990). A subset of researchers examining intimate partner violence over the past few decades have worked to specifically understand both the prevalence and causes of female same-sex IPV (Eaton et al., 2008; 
Glass et al., 2008; Hassouneh \& Glass, 2008; Little \& Terrance, 2010; McClennen, 2005; Ristock, 2003, 2011; e.g., C. Smith, 2011); however much of our understanding of the contexts of and mechanisms underlying FSSIPV is rooted in heterosexist conceptualizations related to men's masculinity and their socially sanctioned dominance and aggression (Davis \& Glass, 2011). Normative heterosexual relationships have historically been characterized by the male member of the relationship being the "partner in control" (Johnson, 1995, 2006). This traditional heterosexual relationship dynamic is rooted in hegemonic masculinity, a gendered normative ideology positioning men as the dominant group in society and a masculinity characterized by power, toughness, aggression, and antifemininity (Levant, Rankin, Williams, Hasan, \& Smalley, 2010) as the authoritative gender role (R. Connell \& Connell, 2005; R. Connell \& Messerschmidt, 2005). The characteristics associated with hegemonic masculinity (e.g., status, economic success, dominance) have been consistently linked to heterosexual men's perpetration of IPV (R. Connell \& Messerschmidt, 2005; Levant et al., 2010; Moore \& Stuart, 2005; Moore et al., 2010; Zurbriggen, 2010). Because these linkages have been demonstrated so reliably in numerous empirical studies, the same heteronormative characterizations of perpetrators of IPV have served as the basis for much of the available research and theory to date related to sexual minority women's perpetration of same-gender IPV (Renzetti, 1992; Ristock, 2002, 2011).

While accounting for the existence of hegemonic masculine norms as underlying causes of women's perpetration of same-gender IPV is justifiable, this framework of same-gender IPV research is conceptually limited in that the analysis tends to focus on women acting as men, rather than on the pervasiveness and consequences of our culture's 
endorsement of and adherence to hegemonic masculinity (C. Smith, 2011). For instance, one of the most common stereotypes ascribed to sexual minority women's same-gender romantic partnerships is that one partner must "play the man" in the relationship (Spaulding, 1999). While this stereotypic assumption is partially based on the visibility of lesbian "Butch-Femme" culture (Spaulding, 1999), it is also rooted in the misogynist, hetero-patriarchal notion that a romantic relationship's legitimacy is contingent upon its adherence to a gender dichotomous dynamic consisting of one masculine (and dominant) partner and one feminine (and subordinate) partner (Spaulding, 1999). The consequence of the widespread application of this particular stereotype, and its implications for the legitimacy of same-gender relationships is that it reinstates a form of male dominance into such relationships (Laird, 1999; Spaulding, 1999). Further, reliance on stereotypes such as this serve more to reify existing marginalizations of systematically minoritized groups (e.g., transgender individuals, women of color, sexual minority women, etc.) than they do to inform hypotheses and intervention work around issues as complex as intimate partner violence. Unfortunately, stereotypes such as the one described above have had a heavy influence on the development and implementation of research and interventions related to female same-sex IPV. Exemplary of this influence, the discourse regarding the role of hegemonic masculinity in the perpetration of IPV remains centralized on maleness and heterosexuality, rather than masculinity as a form of gender identity, expression, and role adherence (Davis \& Glass, 2011). For instance, Eaton et al. (2008) extends the following theoretically-based understanding of IPV as a possible explanation for their findings that power and control relationship dynamics significantly distinguished women with histories of IPV and women with no such histories: 
Gender role theories have traditionally been used; however, without a male perpetrator to define as the abuser, IPV becomes more challenging to explain. Some research has suggested that the abuser in a lesbian relationship is 'borrowing male authority; as this role encompasses dominance and control, and legitimizes power (Kimball, 2001). Accordingly, the abuser in a lesbian relationship may take on qualities that are more typically associated with how the male gender role has been defined by our society. (p. 702)

Because heterosexuality serves as a defining quality of our culture's hegemonic masculine ideology, IPV discourses rooted in stereotypes, like the one described above, unceasingly default to a focus on heterosexual IPV and maintain the systematic silencing of sexual minority women and their experiences with female same-sex IPV (Davis \& Glass, 2011). For instance, in a systematic review and critique of empirical research on IPV among lesbian women and gay men Burke \& Follingstad (1999) consistently compare and contrast results from the studies in their review with results of studies specific to heterosexual IPV:

Lockhart, White, Causby, \& Isaac (1994) reported that, consistent with findings regarding male heterosexual batterers' dependency and need for control (Stordeur \& Stille, 1989), correlational findings indicated that when lesbian partners feel a need to share all recreational and social activities, and express a need to do everything together, abuse is more likely to be part of the relationship. Renzetti's (1988) study, found that abusive lesbians were particularly dependent upon their partners, which also appears true of heterosexual batterers... (pp. 505-506) 
In the above example, the authors provide no initial indication that the review is intended to be comparative between homosexual and heterosexual IPV, yet heterosexual relationship dynamics and IPV correlates are used throughout the review as seemingly default comparison standards. This meta-theoretical framework suggests a prevailing heteronormative focus within the research related to women's use of violence in their same-gender relationships. An especially concerning example of this heteronormative meta-theoretical framework based in stereotypes that serve to further marginalize sexual and gender minorities, is the Lesbian Partner Abuse Scale (LE-PAS-R), a 25-item measure specifically designed to assess IPV victimization among women in same-gender relationships. The LE-PAS-R was developed by McClennen, Summers, \& Daley (2002) with the intention for it to be utilized by practitioners in social work (and potentially related fields) who served sexual minority women. This particular measure is troublesome for several reasons. First, according to McClennen et al. (2002)'s (i.e., the LE-PAS-R's developers) explanation, their primary focus in creating the measure was "...exclusively on designing a measurement scale for identifying variables related to power imbalance between lesbian couples resulting in partner abuse" (p. 279). However, of the 25 items comprising the LE-PAS-R less than one third (eight items; $32 \%$ of all items in the scale) relate to controlling behaviors, while the remaining seventeen items (i.e., $68 \%$ of the scale's items) primarily relate to the abuser's substance abuse and experiences of childhood and adolescent violence. Further, of the six factors defined after three rotations in a factor analysis, none are specific to power and control behaviors. Instead, controlling behaviors are supposed to be reflected in the eight items comprising the first factor, defined as "Communication and Social Skills", while the remaining five 
factors are defined respectively as: Substance Abuse, Intergenerational Transmission of Violence, Fakes Illness, Internalized Homophobia, and Status Differentials. The authors provide in their discussion of results loose explanations for how each of these factors reflect power imbalances leading to intimate partner abuse within lesbian relations, but these explanations are limited at best in that it is simply stated in each factor's definition paragraph that it reflects some dimension of power imbalance and that it has been shown to lead to abuse, yet little-to-no further discussion is provided of (a) how these reflections exist for each factor nor (b) the mechanism relating power imbalance supposedly reflected in each factor directly, or even indirectly, leading to partner abuse within lesbian relationships.

The above-critiqued research process, and the IPV assessment measure developed out of that process, is representative of an epistemic failure to sufficiently attune survey content, language and terminology to the experiences of women in same-gender relationships. The above example further speaks to an overarching failure to adequately investigate the phenomenon of IPV within this population with the save fervor and persistence that has characterized research and action around IPV among individuals in opposite-gender relationships. Importantly, the persistence of heterosexism and the continued marginalization of sexual minorities within scientific research related to intimate partner violence intersects with mainstream feminist history in the United States, and specifically the political climate and prevailing dominant ideologies of the U.S. Second Wave Feminist Movement. The historical analysis below aims to provide an intersectional analysis of the current state of research and social action related to female same-sex IPV. The review will also highlight the need for the specific research questions 
and methodologies present under the current study and the larger program of research in which this study is situated. 


\section{Chapter 2. Contextual Literature Review: Current and Historical Barriers to}

\section{Examining Same-Gender IPV among Women}

The continued state of research specific to IPV among women in same-gender relationships may be indicative of several key issues: (1) continued homophobia and heterosexism within the broader fields of education and research (e.g., Blount, 2006; Brown, Clarke, Gortmaker, \& Robinson-Keilig, 2004; S. J. Ellis, Kitzinger, \& Wilkinson, 2003), (2) the lack of adequate funding for conducting in-depth empirical research on FSSIPV, attributable to various factors (e.g., economic recession, political tensions among marginalized agendas, etc.; Durish, 2011), and (3) continued reliance on or promotion of research and political agendas rooted in the mainstream ideals employed during the U.S. women's and domestic violence movements (i.e., Second Wave Feminism; Gilbert, 2002; McLaughlin \& Rozee, 2001; Ristock, 2001). This study exists as a direct challenge to this latter indicator of the persistence of a heteronormative and oppressive framework in scientific research, policy, and community action-related professional fields. This study's challenge to the barriers created and perpetuated by mainstream feminist agendas is not in service to or an attempt to debunk the work done by the U.S. women's and domestic violence movements. Rather, it is aimed at furthering the endeavor of transforming our current culture of violence into a culture of peace. This endeavor necessitates active inclusiveness of perspectives outside the dominant culture (i.e., white, cis-gender, middle-class, hetero-patriarchy;), which requires a critical understanding of the oppressive structures and cultural norms existing even within social movements and agendas aimed at dismantling oppression (e.g., mainstream traditional feminism in the U.S.; Ristock, 2011). 


\section{Historical Analysis: 20th Century Feminist Perspectives Motivating a Critique of}

\section{Traditional Feminism}

Science has historically been driven by social and political movements and viseversa. For instance, progressions made in the Civil Rights Movement in the U.S. were simultaneously combating against and relying upon scientific theory and evidence to further their campaigns into successes. In the same light, it is important to recognize that there are social and political movements currently happening at the global level that are relevant to the presently present research. That is, this study is a part of a growing body of research and social change action that is simultaneously combating against and relying upon scientific theory and evidence to further the plight of recognizing groups and individuals who identify as members of the LGBTQ community as equal members of society worthy of the same services and rights given freely to those outside of said community (Davis \& Glass, 2011; Lai-Poon, 2011; Ristock, 2001).

Beginning around the 1960s, U.S. Second Wave Feminism and the (anti-) Domestic Violence Movement began gaining considerable traction within the mainstream media and political agendas across the country (Crenshaw, 1991; LeGates, 2001). These joint mainstream feminist efforts began to shift how domestic violence, and the broader concern of violence against women, was defined, understood, and addressed within U.S. politics and research (Bush, 1992; Crenshaw, 1991; LeGates, 2001, Chapter 3; Pleck, 1987). These efforts have been, through the lens of the present analysis, simultaneously means of liberating and oppressing women. The historical analysis that follows aims to elucidate this paradox in order to situate the present research in the context of work that has come before it. 
Regarding the continued effort for women's liberation in this country, U.S.

Feminism has no doubt made considerable strides in establishing women's access to and representation within the various structures and institutions that make up our democracy (e.g., Women's Suffrage achieved during the First Wave of U.S. Feminism, the passing of the Violence Against Women Act during the Second Wave, etc.; LeGates, 2001). However, the benefits of these achievements were primarily experienced by white, middle-class, and presumably heterosexual cis-gender women in the U.S., while women falling outside of that rather narrow identity definition remained under-represented, and in some cases in mainstream U.S. politics and political changes, such as the experiences of African American women in the U.S. South who were systematically discriminated against and effectively denied access to women's (relatively) newly gained voting rights (circa 1913) until the later success of the U.S. civil rights movement during the 1960s and 1970s. In addition to, and intersecting with, issues of continued racism within U.S. feminist agendas, there are some key elements of the U.S. Anti-Domestic Violence Movement with consequences specific to sexual minority women: (1) the assumption of heterosexuality with regard to IPV (i.e., heterosexism; Davis \& Glass, 2011; VanNatta, $2005),(2)$ the continued praise and adherence to the dominant conceptualizations of masculinity (i.e., hegemonic masculinity; Gilbert, 2002; Hassouneh \& Glass, 2008; VanNatta, 2005), and (3) the continued devaluation of femininity (i.e., Antifemininity; R. Connell \& Connell, 2005). Importantly, these issues and their consequences are not simply indicative of oppressive attitudes and beliefs evident at the individual and small group levels, but rather are inherent in the institutional, political, and organizational structures and processes encompassing the overarching field of IPV research, action, and 
service: "Workers and administrators frequently produce normal case categories based on the experiences of those at the top of the social hierarchy - in this case, white, heterosexual, temporarily able-bodied, middle- class, traditionally gendered women." (VanNatta, 2005, p. 417, emphasis added).

The extent to which women's liberation was achieved through Second Wave Feminism is reflected in the movement's thematic mantra, "The personal is political" (Hanisch, 1970), which argues against the idea that women's personal lives are of no concern to the public and political spheres of society. It argues that the personal experiences of women's lives are both relevant to, as well as the result of, contemporary political discourses. However, exemplary of the pervasiveness of an oppressive system is the (lack of) treatment of issues faced by historically marginalized and minoritized throughout the women's and domestic violence movements. Specifically, while women and femininity as a whole have been targets of oppression, so to have groups and individuals whose identities fall outside the boundaries of the hegemonic heterosexuality and the broader white cis-hetero-patriarchal definition of our dominant culture. These groups have been systematically ignored and further marginalized as a function of the political discourses of Second Wave Feminism. The phrase, "the personal is political", brings to light the idea that individuals are subjective actors in social and political discourses and that each individual is both influenced by and influencing public policy. Evidence of this notion is apparent even in the origins of the phrase itself in which Hanisch (1970) expresses remnants of the intersectional systems of oppression at work during this time: 
The groups that I have been in have also not gotten into 'alternative life-styles' or what it means to be a 'liberated' woman. We came early to the conclusion that all alternatives are bad under present conditions. Whether we live with or without a man, communally or in couples or alone, are married or unmarried, live with other women, go for free love, celibacy or lesbianism, or any combination, there are only good and bad things about each bad situation. There is no 'more liberated way; there are only bad alternatives. (p. 4)

The feminist rhetoric reflected in this statement speaks to many different aspects of how women's oppression is historically understood in terms of the split of public/private spheres. Often times, injustices against women occur in spaces that are considered strictly private; however, this mantra argues against keeping such injustices in the private sphere and for women's oppression to be brought to the political forefront. Such an argument overturns the historical interpretation of the objective government and turns the political world into a subjective space in which cases of individual oppression are just as potent as cases concerning a particular group of people. Also, the historical context of this mantra being placed in a time when women's voices were still considered as belonging primarily to the private space of the home and not a part of the political space of society forces the voice of woman to be taken out of its historically understood and accepted level of analysis and into broader levels of the public and political spheres with the hope that more people will see the importance of this voice. That is, the voice of woman has historically been heard on a more individual, and therefore disjointed and varied, level at the expense of the amplification of a unified community and policyleveled voice. The latter voice has instead been simultaneously spoken for and silenced 
by the hegemonic group (i.e., men in power) in ways that work to uphold the dominance of that group. While this specific dynamic of our culture in the U.S. has progressed somewhat in the 45 years since Carol Hanisch unknowingly kindled the flames of Second Wave Feminism (Hanisch, 1970), the processes of oppression and status maintenance remain steadfast in our culture and in our political processes. That is, while women's voices have gained much ground in the public spheres of society and while explicit sexism has become increasingly less socially acceptable, rather than explicitly normative, oppression itself remains a fundamental characteristic of our society's structure and mundane functionality. The feminist spirit motivating the notion that the personal is political (Hanisch, 1970), is indicative of how the feminist work conducted during the $20^{\text {th }}$ century in the U.S. aimed at dismantling the current status quo (LeGates, 2001; C. Smith, 2011), which posited men and masculinity as superior to women and femininity (Johnson, 2006). In doing so, the work of mainstream U.S. feminism that was focused on addressing violence as a form of oppression against women involved upholding certain gender-based stereotypes (e.g., notions that women are innately chaste and men innately promiscuous) in efforts to bring attention to the very serious social problem of intimate partner violence (Johnson \& Ferrero, 2000; VanNatta, 2005). These gender-based stereotypes were rooted in the pervasive notions of heteronormativity and the gender binary. The guiding rhetoric of this movement allowed little-to-no room for the recognition of variations in gender identities and ascribed roles such that women continued to be understood only in terms of their degree of femininity, and men in their degree of masculinity: 
The gender called man, culturally defined in its manhood, often needs to reject the gender called woman, itself culturally defined in its womanhood, by distinguishing itself as strong, powerful, controlling, and often aggressive and violent. ... 'If, in men's accounts of aggression, we are told what it is like to take control, in women's accounts we hear about what it means to lose control. For women, the threat comes from within; for men, it comes from others. For women, the aim is a cataclysmic release of accumulated tension; for men, the reward is power over another person, a power that can be used to boost self-esteem or to gain social and material benefits. For women, the interpersonal message is a cry for help born out of desperation; for men, it is an announcement of superiority stemming from a challenge to that position'. (Campbell, 1993, pp. 7-8; Quoted in Gilbert, 2002, pp. 1274-5)

\section{Intersections among the Gender Dichotomy, Patriarchy, Heterosexism and Interpersonal Violence}

Feminists who brought the focus to domestic violence in the 1970s viewed the victim as someone without blame, the problem as one of men abusing women, and the violence as a component of a patriarchal system (Berns, 2004). (C. Smith, 2011, p. 131)

Scientific research and its practical applications have historically defined biological sex and gender identity according to a dichotomous structure of male/masculine and female/feminine, and this structure has served as the basis for discourses around gender identities and the gendering of social roles and behaviors (Auster \& Ohm, 2000; Barry, Bacon, \& Child, 1957; Bem, 1981b; Constantinoble, 1973; 
Lenney, 1991; O’Neil, 1981; Pleck, 1987; Spence \& Helmreich, 1980; Terman \& Miles, 1936). Characteristics commonly associated with the feminine side of the gender binary system include nurturance, frivolity, ignorance, uncontrolled emotionality, hysteria, physical and emotional weakness, and above all subordination to men and masculinity (Bargad \& Hyde, 1991; Bem \& Lewis, 1975; Hassouneh \& Glass, 2008; Levant, Richmond, Cook, House, \& Aupont, 2007). Conversely, the masculine side of this binary is characterized by toughness and aggression, stoicism and restricted emotionality, independence and self-reliance, social dominance and authoritarianism, and above all the avoidance of femininity (Auster \& Ohm, 2000; Bem, 1981b; R. Connell \& Messerschmidt, 2005; Levant et al., 2010; Pleck, 1987). Social and natural scientists alike have often considered this gender binary system as biologically determined in that masculinity and femininity, gendered characteristics, are typically considered as tied to male and female, or sex characteristics. Further, the conflation of sex and gender, as well as the espoused binary structure of these constructs, is typically assumed to be relatively static across time, place, and subject. That is, babies assigned as female at birth are expected to possess "feminine" qualities and those assigned as male are ascribed "masculine" qualities, and this expectation is based on the assumption that these qualities are naturally inherent in females and males (Barry, et al., 1957; Bem, 1981b; Constantinoble, 1973; Lenney, 1991; Pleck, 1987; Spence \& Helmreich, 1980; Terman \& Miles, 1936). Indeed, dichotomized gender differences are, generally, seen as the starting point for understanding differences among individuals within a given population.

Some researchers and activists have worked throughout the U.S. Women's and Domestic Violence to challenge the mainstream notion of a gender binary and the 
assumption of such strict biological determination. Over the past couple of decades, this challenge has gained a considerable foothold in the overarching conversation around gender identity and gender role adherence within scientific research and its practical applications. Theory in this respect has argued that the traditional notion of a static binary structure of gender produces a reductive and ultimately incomplete understanding of individuals and human behaviors. Challengers to the gender binary further argue, and in many cases empirically demonstrate, that individuals' gender identities and their behaviors are likely the result of a complex mixture of biological and genetic determinants combined with the socialization of behaviors, attitudes, and beliefs throughout the course of human development (R. Connell \& Messerschmidt, 2005; Levant \& Richmond, 2007; Levant et al., 2010). This theory acknowledges that individuals experience and express gender in a multitude of ways that are not necessarily consistent with the definitions of gender identity and gendered characteristics prescribed under a dichotomous framework of gender (Bem, 1981b; R. Connell \& Connell, 2005). The theoretical evolution of gender, as a construct, has led to the notion of a, still singledimensional, spectrum of gender identities, with masculinity and femininity at either end, which acknowledges variation in how individuals identify with, express, and experience gender (Bem, 1981b; Wetherell \& Edley, 1999). In response to this notion of a gender spectrum, researchers have considered that perhaps gender exists along more than a single dichotomous dimension of male/masculine and female/feminine. Sandra Bem (1977) developed the Bem Sex Role Inventory (BSRI), a measure that positions gender identity and expression along a multidimensional plane with four defined categories of gender role adherence: masculine, feminine, androgynous (i.e., high feminine and high 
masculine), and undifferentiated (i.e., low feminine and low masculine; see Figure 1). In more recent years, discourses around gender have begun to posit that gender identity can take on a multitude of dimensions that are not necessarily mutually exclusive, linear, nor strictly categorical with clear definitions of boundaries and characteristics (Auster \& Ohm, 2000; Carrigan, Connell, \& Lee, 1985; R. Connell \& Messerschmidt, 2005; Pleck, 1987). Advances in this emerging framework necessarily challenge how we use language as a way to categorize gender. This is especially true with regards to a trend in sociopolitical discourses and scholarship that seems to be tentatively moving toward a more fluid understanding of gender identity and expression. The use and conceptualization of gendered language, then, could be expected to evolve in a parallel fashion in order to reflect changes in the experiences we describe with language. However, while many researchers, practitioners, and theorists acknowledge and endorse an understanding of gender existing as a construct defined in multiplicities (i.e., without strict categorizations and boundaries), commonplace language related to gender remains largely bound by the traditional assumption that gender exists in a dichotomous structure and the colloquialisms associated with this assumption. That is, despite efforts by researchers and activists to be inclusive of the multitude of gender identities, efforts to incorporate the notion of multiplicity into our common use of language when talking about gender have yet to overcome the traditionally accepted and espoused binary understanding of gender.

The "problem" of lesbian battering. The perpetuation of a gender binary framework has heavily influenced IPV-related research and intervention and prevention strategies in that this ubiquitous nature of this framework has culminated in the creation of "the typical victim" and "the typical perpetrator" of IPV (VanNatta, 2005). The notion 
that women are victims and men are perpetrators became increasingly popular throughout the latter half of the 20th century, to the point that the vast majority of domestic violence services developed through these movements was modeled according to this dichotomy. The victim-as-woman and perpetrator-as-man dichotomy lead to the stereotyping of these roles based on gender role stereotypes associated with either side of the male (masculine)/female (feminine) dichotomy. While "victim" became associated with stereotypically feminine qualities such as weakness, disempowerment, and dependency; "perpetrator" became associated with opposing masculine stereotypic qualities such as dominance, power, and monster. The resulting popular attitude around domestic violence is one that prohibits acknowledging the existence of women's violence. Further, because the qualities of perpetrators involve characteristics such as dominant and monster, which were particularly intentionally attributed only to men, women's violence toward other women is so far further removed from the allowed discourse that it becomes unfathomable. As such, the reductive semantics and rhetoric encompassing the mainstream understanding of intimate partner violence allowed for devastating continued ignorance of same-gender IPV among women, and has resulted in the further marginalization of sexual minority women. Further, this continued ignorance was not always passive and the attitudes driving this ignorance were not always implicit among some of the most influential voices of Second Wave Feminists (e.g., Betty Friedan), and the collective influence of those particular voices has successfully maintained the status quo characterized by and in the overall continued dominance of hegemonic masculine heteronormativity (LeGates, 2001). 


\section{Gender role stereotyping and myths about women, femininity, and lesbian}

relationships. Stereotypes, particularly those attributed to marginalized populations, often have much more devastating consequences than their seemingly benign colloquial meanings might suggest (Hassouneh \& Glass, 2008; LeGates, 2001). The rhetoric of feminist and domestic violence movements during the 1970s through the remainder of the 20th century has resulted in stereotypes and myths about women, femininity, and lesbian relationships that impact the definition, measurement, and response of FSSIPV. These colloquial stereotypes have led to a rhetoric that reduces woman-to-woman violence to "cat fights", or harmless and/or frivolous interactions among women (Hassouneh \& Glass, 2008; Merrill, 1996). Most exemplary of such stereotypes ascribed to sexual minority women is the myth of the "lesbian utopia", which, promulgates the notion that women are non-violent and nurturing, and therefore their intimate relationships must be an exaggeration of those qualities (Elliot, 1996; Hassouneh \& Glass, 2008; McLaughlin \& Rozee, 2001; Ristock, 2002). Additionally, the stereotypic notion of the "butchfemme" composition of lesbian romantic relationships (i.e., wherein one romantic partner is highly masculine in appearance and behavior, and the other highly feminine) serves as a particularly insidious mechanism in which the gender dichotomy inherent within hegemonic masculine heteronormativity maintains the status quo by promoting the assumption that, even outside of a heterosexual context, masculinity and femininity must exist as polar opposites. The origins and continued promotion of stereotypes such as these, as well as the dichotomous notions around gender and violence permeated in part by the U.S. women's movement(s), bring to light the primary barrier to recognizing, responding to, and ultimately preventing same-gender IPV among women: the "problem" 
of women's perpetration of same-gender IPV for feminist agendas. That is, acknowledging the existence of FSSIPV as more than a rare phenomenon perpetrated and experienced by a "few bad apples" is in direct opposition to promoting the very ideas and gendered rhetoric that some of the most influential feminists developed and relied upon to ensure the success of the movement(s). Thus, IPV in women's same-gender relationships has been seen by some not as a social problem worthy of adequate acknowledgment and intervention, but rather as a politically logistical problem in need of cover up and silencing in public discourse (LeGates, 2001).

While the work of the Feminist Movement in the U.S. during the latter half of the 20th century was instrumental in dismantling the notion that IPV is a private issue strictly occurring in and affecting the domestic context, there exist holes in this work that have had detrimental consequences, specifically for marginalized populations of femaleidentified groups and individuals. Further, much of the rhetoric characteristic of these movements has resulted in the permeation of stereotypic ideologies around IPV that promote a dichotomous framework with regards to gender and the characteristics of perpetrators and victims of IPV. Given this analysis, it is important to note that the continued dearth in the research related to FSSIPV is in part attributable to this reductive rhetoric employed by highly influential mainstream U.S. feminists during the latter half of the 20th century. This rhetoric is not assumed to be fabricated by the U.S. feminist movement(s), but rather, a result of a substantial history of our collective adherence to hegemonic masculine and heterosexist norms and ideals that serve to maintain the underlying structure of our dominant white hetero-patriarchal culture. The present research identifies hegemonic masculine heteronormativity, and the underlying 
oppressive structure it supports as a root cause of intimate partner violence as a whole, and particularly of IPV that occurs among marginalized populations. This assertion is rooted in a feminist framework aimed at promoting deeper awareness and criticism of a seemingly benign and innate, but more likely toxic and socially constructed, dichotomous structure of gender identity and sexuality. Evidence of the reductive nature and negative consequences of this structure are discussed in the following description of the current state of intimate partner violence research, with specific attention given to research on same-gender IPV among women.

\section{The Current State of Estimating the Prevalence of FSSIPV}

Research on violence against women has exploded in the past 20 years, particularly in the areas of intimate partner violence and sexual assault. Despite this outpouring of research, many gaps exist in our understanding of violence against women. For instance, reliable information on minority women's experiences with violence is still lacking. (Tjaden \& Thoennes, 2000a, p. iii)

Over the past few decades, intimate partner violence victimization survey data has been collected at a range of population levels including national, states, and local municipalities in the U.S. Findings based on these data are typically been made publicly available through reports providing summative and conclusive information on the rates, prevalence, and impact of intimate partner violence, often with distinctions made based on region, race, ethnicity, age, income, and education (M. C. Black et al., 2011; M. J. Breiding, Black, \& Ryan, 2008; Tjaden \& Thoennes, 2000a). While immensely informative and necessary to IPV-related research and intervention and prevention work 
overall, these inquiries have, until quite recently, utilized operational definition(s) of "minority women" that do not incorporate women in same-gender intimate relationships.

National Violence Against Women Survey (NVAWS). Despite acknowledging the relatively overwhelming amount of research on violence against women (quoted above), the full report resulting from the National Violence Against Women Survey (NVAWS) also indicates in the summary of the data collection methods that questions regarding perpetrator characteristics would generate data valuable in making inferences about sexual minority women and same-gender IPV among women. That is, femaleidentified respondents were asked if they had experienced abuse by anyone, male or female. However, quick analysis of the questions in this survey makes it clear that the inquirers were primarily, and potentially exclusively, interested in heterosexual male-tofemale intimate abuse. Female respondents were asked the following two initial questions regarding intimate partner and sexual abuse:

(1) Has a man or boy ever made you have sex by using force or threatening to harm you or someone close to you? Just so there is no mistake, by sex we mean putting a penis in your vagina?

(2) Has anyone, male or female, ever made you have oral sex by using force or threat of force? Just so there is no mistake, by oral sex we mean that a man or boy put his penis in your mouth or someone, male or female, penetrated your vagina or anus with their mouth. (p. 4)

While inferences might have been possible with this second question regarding the rates and prevalence of female-to-female sexual abuse, the emphasis on men and boys as the potential perpetrators frames the question in a primarily heteronormative context 
and therefore decreases the likelihood of eliciting unbiased survey responses from all respondents. For example, a female respondent may have answered "no" to the second question despite having experienced physically forced and/or coercive (via threat of force) oral sex by a female partner, while respondents who answered "yes" could have been referring to a female perpetrator, but the abusive act in question did not fit the narrowly defined criteria provided in the question's second "clarifying" statement and so any subsequent questions may have been answered in reference to a male perpetrator in order to fit the researchers' definitions.

This issue is quite consistent throughout the report's presentation of the survey's methodology and findings. Incidentally, analysis of data collected through the NVAWS was presented in a separate publication in the year prior to the official release of the full report (Tjaden, Thoennes, \& Allison, 1999) compares rates of intimate partner victimization among same-sex and opposite-sex couples. Tjaden et al. (1999) attempt to explore possible correlates of IPV victimization among female respondents who indicated their sexual orientation as lesbian, and report that lesbian-identified women experience significantly higher rates of IPV and sexual violence victimization than heterosexual women, regardless of the perpetrator's gender. However, the authors provide an important caveat that lesbian identified respondents who affirm IPV victimization experienced such victimization by male intimate partners. Thus, the interpretation of this finding is in service to the notion that intimate partner violence most often occurs in a heterosexual male-to-female context. Further, these interpretations add to the notion of a "stereotypical battered woman", or a "typical victim" as discussed earlier, which deflects from the 
phenomenon of interest (i.e., IPV among sexual minority women), and consequently distracts researchers and activists from addressing this phenomenon.

National Intimate Partner and Sexual Violence Survey (NISVS). In the decade since the release of the NVAWS (Tjaden \& Thoennes, 2000b) and subsequent publications (e.g., Messinger, 2011) several advances have been made to promote the recognition of IPV in same-gender relationships alongside the various efforts made to increase LGBTQ individuals' equal access to basic human rights (e.g., efforts to ban discrimination against LGBTQ individuals in the workplace, homelessness intervention strategies that specifically target LGBTQ homeless youth, the successful movement for equal marriage rights for same-sex couples, etc.). As previously mentioned (see Problem Statement), Walters et al. (2013) provide insight into the rates, prevalence, and correlates of IPV distinguished by individuals' sexual orientation in a secondary report augmenting the findings presented in the 2011 Full Report of the National Intimate Partner and Sexual Violence Survey (NISVS; M. C. Black et al., 2011). The NISVS report provides more recent nation-level information on the rates, prevalence, and impacts of IPV victimization, and was conducted using similar sampling and data collection methodologies as the NVAWS with additional survey questions targeting information about perpetrators of IPV from respondents who affirm having experienced IPV victimization. The NISVS Full Report details differences in IPV victimization prevalence and impacts based on race, ethnicity, and age, and provides limited such information regarding differences in sexual orientation among respondents. Walters et al. (2013), however, provide a much deeper analysis into these differences in their secondary report of findings from this survey. While the findings presented by Walters and colleagues are 
invaluable to this study and IPV research as a whole, there are some notable methodological limitations and concerns within this secondary report. Foremost of these concerns is the simple fact that this report comes approximately two years following the release of the NISVS 2011 full report (M. C. Black et al., 2011), which only a minimal analysis of IPV prevalence rates based on sexual orientation.

Oppression and violence at the intersections of gender, sexuality, race, class, and scientific inquiry. As previously mentioned, the original full report of the NISVS does provide a comparatively more in depth distinguishing analysis of IPV victimization rates based on race, ethnicity, and sex (male and female), all of which constitute common distinctions made in empirical studies across most fields of social scientific research (Sidanius \& Pratto, 2001). However, the focus on these three factors alone in the current empirical IPV literature indicates systemic ignorance to the fact that a person's identity is comprised of a multitude of characteristics not necessarily defined solely by race, ethnicity, and sex as mutually exclusive components of identity. This implies the assumption that experiences of oppression and marginalization occur exclusively according to such broadly stroked distinctions, regardless of diversities that exist within these three categories and among other identity characteristics such as sexual orientation (Crenshaw, 1991; C. Smith, 2011). This methodological limitation perpetuates an underlying assumption that a mutually exclusive relationship exists between one's racial, ethnic, socioeconomic, generational, gender, and sexual orientation identity components. While such broad stroke categorizations may serve as convenient ways to gather, interpret, and report data and analysis results, this methodological framework also reinforces reductive frameworks, such as the gender binary discussed above, that have 
historically served to maintain a particular social hierarchy and status quo. Importantly, this reductionist methodological framework results in a substantially limited understanding of IPV perpetration that overlooks the multilevel and systematic processes, correlates, and causes of this phenomenon. A similar consequence is apparent in the ways mainstream U.S. feminist movements have neglected to engage in an introspectively intersectional analysis of the influence of patriarchy and white women's political agendas on the continued structural, institutional, and interpersonal oppressions perpetrated against people of color, LGBTQ groups and individuals, people with physical disabilities and mental illness, and working class and poor individuals of all genders (Crenshaw, 1991; LeGates, 2001; Ristock, 2011). These overarching limitations in research and political activism culminate in the perpetuation of a siloed approach to addressing social justice issues and public health concerns (e.g., intimate partner violence). This siloed approach has defined mainstream research and politics in the U.S. over the past century and a half.

\section{The "Coming Out" of Same-Gender IPV among Women}

In response to the above described paradoxical state of mainstream feminism's recognition and response to same-gender violence among women, those scholars and activists working throughout the women's movement to bring attention to the issue have characterized their work along the lines of "coming out" about this phenomenon. Researchers and activists exemplifying this characteristic begin their arguments, and often title their writing, in such a way that speaks to the continued silence around IPV among women in same-gender relationships using phrases such as: "Invisible victims..." (Messinger, 2011), “Letting out the secret...” (Lockhart et al., 1994), "No more 
secrets..." (Ristock, 2002), "Nowhere to hide...” (Balsam, 2001), “Shattering illusions..." (Elliot, 1996), "Naming the violence..." (Lobel, 1986), and "There's no pride in domestic violence..." (Duffy, 2011). The logic behind many of these phrases may be understood as rhetorical devices used to call out the continued lack of attention given to this particular phenomenon within the broader academic and service providers' networks (e.g., counseling, crisis line, and shelter providers). However, the trend of opening conversations and discourses in this particular way also speaks to a more implicit notion of shame often experienced by sexual minority women, as well as survivors of IPV. The combined impact of the shame experienced by sexual minority women who are also survivors of abuse is most vividly exemplified in the latter of these phrases, "There's no pride in domestic violence..." (Duffy, 2011). Here, the author invokes both the colloquial notion "coming out of the closet" often used by /or in reference to members of the LGBTQ population, as well as the history of not only shaming survivors of IPV, but also of silencing the issue of IPV as a whole by denoting it as a "private issue" not appropriate for public discourse. Collectively, framing discourse about same-gender IPV among women reinforces the overall subordination of women and their experiences and ultimately cloaks the diversity of those experiences under the guise of "women's issues". This guise has historically served as a smokescreen-like strategy for deliberately overlooking and/or dismissing women and their experiences in order to uphold the patriarchal hierarchy underlying our society's structure.

\section{Patriarchy, hegemonic feminism, and intersectional marginalization.}

Women's oppression has been historically understood within a white, middle-and-upperclass, heteronormative, and cis-sexist context. The rhetoric of first-wave feminism's most 
influential voices, which were primarily focused on women's suffrage, often deliberately silenced the voices and perspectives of women of color (LeGates, 2001). Similarly, second-wave feminism, while making some strides against the white-supremacist biases of the previous movement, continued a tradition of intersectional marginalization in which adherence to traditional femininity (i.e., primarily heterosexuality, whiteness, chastity, passivity, and aversion to violence) defined a "good" feminist and a woman entitled to the rights fought for during this movement (Friedan, 1998; LeGates, 2001; Newton, 1984). A "good" mainstream second-wave feminist woman would simultaneously minimize her own femininity by altering her feminine dress (e.g., restricting her wardrobe to consist only of slacks and button-downs in muted colors) and behavioral tendencies (e.g., compassion - often perceived as nurturance and weakness and displays of emotions - often perceived as hysteria). She would resist patriarchy while simultaneously upholding patriarchal values and institutions such as the "traditional family" (strictly heterosexual and presumed to be white), capitalism and competition, and individual entitlement at the expense of others (e.g., people of color, LGBTQ people, poor people, etc.). She would also only be associated with domestic violence as either a survivor or a friend of a survivor, but certainly not a perpetrator. A survivor would exhibit strength in her escape (and would not question the notion that leaving is the only option) or would be so overcome by her abuser that she needs someone else to think for her. Outside of this "good" second-wave feminist box exists the groups and individuals who defy, in some way or another, this movement's leaders' collective definition of a "real woman" and a "real person". Individuals within this group are those whose past, current, and potential needs related to IPV victimization have been neglected. Furthering 
this neglect are the colloquialisms used to described IPV by scholars (in any relevant academic field including psychology, public health, sociology, law, criminology, etc.), community-based service providers (particularly advocacy training providers), and policy makers. These colloquialisms follow a rhetoric that is further marginalizing of people outside of the second wave feminist box by proclaiming that, "while anyone can be a victim, survivor, or perpetrator of IPV, most of the time it is a male perpetrator against a female victim or survivor". Further, when women's violence is identified, it is all too often reduced to playfulness or "cat-fighting" and thus is neither recognized as legitimate violence nor as symptomatic of a larger social issue (i.e., macro-and-micro-level oppressions committed in the name of Patriarchy and maintaining the status quo). Such an oversight, whether unintentional or deliberate, has blockaded efforts made to effectively prevent IPV in our society, including heterosexual (male-to-female) IPV. This oversight is exemplary of how women whose identities fall outside of hegemonic feminism (i.e., white, cis-gender, middle-class, heteronormative feminism) have experienced unique oppressions perpetrated simultaneously by white-hetero-patriarchy and mainstream feminism.

The above-described forms of oppression perpetrated against marginalized populations, and particularly those perpetrated against sexual minority women, and the barriers to intervening and preventing intimate partner violence that occurs among sexual minority women necessitate a research approach that takes into account this unique context. As such, a working intersectional model predicting the perpetration of samegender IPV among women, depicted in Figure 2, was developed to guide the design, analytic approach, and conclusions for this study, as well as future projects planned under 
the overarching research program in which this study is situated. In the sections that follow, I will detail the constructs within the working intersectional model of depicted in Figure 2 as they pertain to the theoretical, contextual, and historical analysis provided above. I will then detail this study's methodological and analytic approaches, followed by this study's findings and conclusions. Finally, I will discuss the implications of this study's findings both in terms of immediate possible applications, as well as the next steps and future directions in this overarching program of research. 


\section{Chapter 3. Conceptual Literature Review: Overview of a Working Intersectional}

\section{Model Predicting Female Same-Sex IPV}

The working intersectional model depicted in Figure 2 model is conceptualized in order to allow for a systematic analysis of the structural, institutional, and individual factors and mechanisms underlying same-gender IPV among sexual minority women. This model was developed following the deconstructive theoretical lens provided by R. M. Smith, Parrott, Swartout, \& Tharp (2015)'s analysis of hegemonic masculine domains and heterosexual men's perpetration of sexual violence toward their intimate partners (see Figure 3). However, the working intersectional model serves as a guide for expanding R. M. Smith et al. (2015)'s analysis of IPV perpetration by accounting for the unique experiences of sexual minority women.

The primary construct domains presented in the working intersectional model as predictors of same-gender IPV among women include endorsement of hegemonic masculinity, adherence to hegemonic masculine gender roles, experiences of stress related to adhering to those normative gender roles, and experiences of stress due to experiencing oppression(s) based on one's minoritized status. To date, research specific to any of these construct domains has been primarily focused on understanding and explaining heterosexual (male-to-female) IPV, with very little empirical work done to examine the relations among these constructs and IPV outside of heteronormative contexts. Despite this, research relating gender role adherence and gender role stress has been reported and applied in various settings under the assumption that findings from such research are representative of the general population, regardless of differences in sexual orientation or trans and non-trans gender identities. These hetero- and cis- 
normative assumptions are particularly evident in the research frameworks underlying the existing survey measures designed to capture the working intersectional model's genderrelated constructs. Specifically, research related to the influence(s) of gender role adherence and gender role stress on IPV perpetration often deliberately excludes LGBTQ individuals or fails to assess those components of identity among research participants (Vance \& Green, 1984). For instance, the Bem Sex Role Inventory (BSRI; Bem, 1981a) was developed and validated using a sample for which the only demographic characteristic available is whether or not participants identified as male or female (Bem, 1977), but still this measure has been widely used since its development with heterosexual and LGBTQ populations of diverse racial and ethnic backgrounds under the assumption that this measure's adequacy in assessing gender identity is not influenced by respondents' sexual orientation or other components of their identities not defined by their birth-assigned sex. This reductive assumption disregards sexual and gender minorities' unique experiences of systemic discrimination, and the potential influence of those experiences on LGBTQ individuals' gender identity development and expressions (O’Neil, 2008, p. 425). The current research intends to specifically address this limitation by evaluating the construct validity and content coverage of survey measures designed to assess each of the constructs present in the working intersectional model with specific attention to the measures' relevance to sexual minority women's identities and experiences. Each of the constructs present in the working intersectional model are detailed below in terms of the historical, theoretical, and empirical development of each construct and its relevance to the current study. 


\section{Minority Stress}

Minority stress encompasses a wide range of experiences related to an individual or group's status as not being a member of the dominant culture (K. W. Allison, 1998; DiPlacido, 1998; Link \& Phelan, 2001; I. H. Meyer, 2003). In general, the dominant culture can be understood as being one or both of the following: (1) representing the majority of the population, and therefore understood and referred to as the "normal" standard to which all others (i.e., cultural minorities) are compared, and (2) representing and referred to as the "normal" standard by virtue of some achieved and maintained status quo, regardless of the proportion of groups and individuals that in fact are represented by this cultural definition (Brooks, 1981; Sidanius \& Pratto, 2001).

As has been previously detailed, sexual minority women have, and continue to be, subjected to oppressions perpetrated against them across all levels of analysis. The structural and institutional marginalization of sexual minority women is perhaps most clearly visible in terms of the intersectional marginalization and intentional systematic silencing of sexual minority women throughout the U.S. Women's and Domestic Violence movements. For instance, Marlene LeGates (2001) notes that,

Betty Friedan, who wanted a broad-based, mass movement that would include churchwomen along with welfare mothers, described the lesbian issue as a 'sexual red herring' that would sidetrack the movement and turn off 'those $8,000,000$ woman's magazine readers.' (p. 362).

Prior to and paralleling with Friedan (2013)'s public homophobic and heterosexist proclamation, lesbian and bisexual women were central to the work of Second Wave Feminism, also striving for a "broad-based, mass movement" but one that would 
challenge the hegemony of church women, middle-class women, white women, and heterosexual women claiming the title of "feminists". While Betty Friedan was positioning herself as an authority of U.S. Feminism's Second Wave, black lesbian feminists were taking their social action into white bars to promote the desegregation of such supposedly "public spaces" and racially diverse groups of working class and welfare-receiving feminists were working to address police brutality and discrimination and state-maintained economic oppressions (LeGates, 2001). Yet, what prevailed among these various social change efforts during the 1950s and 1960s were the feminist agendas that maintained the status quo and superiority of white, middle-class, heterosexual women. As a result, mainstream feminism moved forward in its second wave as an institution that sanctioned and at times promoted the racist heterosexist hegemonic paradigm that is central to maintaining the dominance of patriarchy and the constant marginalization of groups and individuals who challenge that dominance. Given this historical context and analysis, this study will explore the potential influence of minority stress among sexual minority women through an intersectional lens allowing for a multifaceted understanding of the historical, structural, situational, and individual factors contributing to IPV perpetration within this population.

The majority of available theoretical and empirical work to date has tended to focus on singular facets of minority stress (e.g., internalized homophobia) or outcomes related to minority stress (e.g., substance abuse) within the LGBTQ population when examining the potential causes and correlates female same-sex IPV perpetration (e.g., Balsam \& Szymanski, 2005). Thus, the analysis throughout this body of research remains incongruent and limited both in generalizability and in scope of analysis. For example, 
many studies examining the negative health disparities experienced by LGBTQ people as a function have consistently found that substance abuse is highly prevalent within this population, and the majority of known descriptive, causal, and correlational studies seeking to identify correlates and causes of same-sex IPV have found direct and indirect links between the presence of alcohol or drug abuse and the existence of IPV among lesbian couples (Burke \& Follingstad, 1999; Renzetti, 1998). However, the emphasis on substance abuse alone among LGBTQ people, and in particular among sexual minority women, often distracts from the likelihood that minority stressors may be the underlying causal factors for both substance abuse and the perpetration of IPV among members of this population. Balsam \& Szymanski (2005) provide one of the first and only systematic empirical examinations of the role of minority stress in women's perpetration and victimization of same-gender IPV in which sexual minority stressors including internalized homophobia and stigma consciousness (further described below) were assessed and analyzed in terms of their influence on participants' reported relationship quality and satisfaction with their same gender intimate partners. Relationship quality was in turn examined as the mediating factor through which internalized homophobia would predict participants' perpetration and victimization of recent (i.e., within 12 months of participating in the study) same-gender IPV. While the authors found support for the above described hypothesized mediation model (see Figure 4), their analysis remains substantially limited with regard to its ability to speak to how sexual minority stressors (i.e., internalized homophobia and stigma consciousness) might intersect with additional facets of an individual's identity and experiences of same-gender IPV. For instance, while the authors control for differences in race, income, and education levels, 
in their analysis examining the possible correlates of FSSIPV, the sample utilized for this analysis is primarily representative only of the dominant culture (i.e., 85\% European American, $76 \%$ with a college degree or higher level of education, and $51 \%$ with a household income greater than the national median income for 2005; DeNavas-Walt, 2010). As such, the current project will work to take into account the possible ways in which women's experiences of multiple forms of oppression based on aspects of their identities not characterized by sexuality (e.g., race, ethnicity, class, gender identity, age, religion, and spirituality) intersect with one another and how those intersections relate to FSSIPV.

This study's focus on minority stressors specific to sexual minority groups (described below) is motivated by the goal of developing a comprehensive and practical understanding of the mechanisms underlying sexual minority women's perpetration of same-gender IPV. Thus, three existing survey measures designed to assess levels of sexual minority stress will be evaluated in this study in terms of their accuracy in assessing the range of experiences related to minority stress among sexual minority women: (1) the Lesbian Internalized Homophobia Scale (Szymanski \& Chung, 2008), (2) the Outness Inventory (Mohr \& Fassinger, 2000), and (3) the Stigma Consciousness Questionnaire (Pinel, 1999). The primary constructs represented in these measures are further described below and the of each measure relevance to this study is shown in Table 1.

Heterosexism. The discourse of violence against women has largely been within the framework of heteronormativity and under the pretext that there exists a gender binary (Davis \& Glass, 2011). Research and political discourses in current literature and 
socio-political contexts challenges these assumptions and allows room for the voices of those previously silenced under this pretext. As a result, research related to nonheterosexual couples' and individuals' experiences has suffered over the course of the movement against intimate partner violence. This is problematic given that sexual and gender minorities have much different experiences than heterosexual cis-gender groups and individuals, and have differences within and among groups of different sexual and gender minorities: "The use of heterosexual models to explain lesbian IPV ignores the diversity of experiences both within and between both groups" (Davis \& Glass, 2011, p. 17). Research on the topic of minority stress has, to some degree, acknowledged, and looked into the effects of, the phenomenon in which minority groups are talked about in terms of how their behaviors and experiences differ from those of the dominant culture. This has largely been done with regards to ethnic and racial minorities situated in cultures dominated by white patriarchal culture (e.g., the United States and European nations). In the same respect, the experiences of LGBTQ groups and individuals have been compared to the heterosexual world in such a way that suggests that heterosexuality is the norm, and identifying any other way is somehow deviant (or the use of the phrase "gender dysphoria" to classify the experience of not identifying with one's assigned gender at birth; Ault \& Brzuzy, 2009; e.g., the inclusion of homosexuality as a disorder in the Diagnostic and Statistical Manual of Mental Disorders; Silverstein, 2009). Such discourses leave very little room for speaking to within group differences, as these discourses have a strong tendency to "lump" together individuals regardless of these individuals' and groups' varying experiences, behaviors, and normalities. 
Homophobia. Common themes within the literature related to defining homophobia include (but are not limited to): (1) an irrational fear of homosexuals, (2) a fear of being perceived as (or associated with) homosexual, (3) a fear of homosexuality disrupting the status quo (Herek, 2004; Hudson \& Ricketts, 1980; Kimmel, 2004). These definitions are broad and malleable given the state of LGBTQ politics both in the formal political sense as well as in how we define homophobia in a social or colloquial sense. In other words, these definitions of homophobia serve different agendas (e.g., legal and political versus social comfort/understanding; Herek, 2004). In addition, these definitions provide a way of understanding the difference between homophobia and heterosexism. Specifically, homophobia is defined, regardless of philosophical and applicable characteristics, as specifically targeted at a particular population, whereas heterosexism is a more implicit form of discrimination that stems from the normalization of a particular set of characteristics. Homophobia has multiple manifestations, the most apparent being (1) experiences of homophobia from external sources (aka: "external homophobia") and (2) the internalized experience of oppressive attitudes and beliefs surrounding homosexuality and/or non-heterosexual sexualities (e.g., bisexuality, pansexuality; aka: “internalized homophobia”; Hudson \& Ricketts, 1980; Shidlo, 1994).

External homophobia and stigma consciousness. Much research related to the experiences of individuals and groups who identify as members of the LGBTQ community has focused on the experiences of external homophobia (i.e., experiences of discrimination, harassment, and violence based on one's sexual orientation) and sexuality-based stigma consciousness (i.e., the active and often constant awareness of one's marginalized status and potential for experiencing discrimination, harassment, and 
violence based on one's sexual orientation). For instance, much research has indicated that many health disparities experienced by LGBTQ groups and individuals can be attributed to the experience of external homophobia (Balsam \& Szymanski, 2005). These disparities include (but are not limited to): social isolation, poor social adjustment, depression, suicidal ideation, suicide, increased anxiety, and wishing and between group interpersonal violence (Balsam \& Szymanski, 2005; Mays \& Cochran, 2001). While this research has successfully provided evidence for a causal link between external homophobia and these health disparities, little research has examined the effects of external homophobia, as a form of minority stress, to the experience of intimate partner violence, both on the perpetration and victimization sides (regardless of gender identity). Internalized homophobia. As is outlined above, research related to the experiences of LGBTQ communities has done much work to outline the causes and effects of violence between heterosexual communities and the LGBTQ community (i.e., external homophobia). However, the construct of internalized homophobia, as it relates to interpersonal violence is less apparent in the current literature. In particular, it is unclear whether or not the experience of internalized homophobia has a direct causal link to intimate partner violence among sexual minority women (Balsam \& Szymanski, 2005; Girshick, 2002; Renzetti, 1998). Some empirical findings suggest that experiences of shame, social isolation and increased dependency resulting from internalized homophobia may account for part of the mechanisms underlying women's use of violence toward their same gender intimate partners (Balsam \& Szymanski, 2005; Renzetti, 1992), while other findings suggest that internalized homophobia is associated with internalized victim blaming among survivors of female same-sex IPV (Girshick, 2002). Further, if there is a 
link between internalized homophobia and interpersonal violence, more systematic research is needed to determine if this relationship extends to intimate partner violence, and if so, to what degree do these experiences influence the rate of perpetration and/or victimization of IPV within same-sex couples?

\section{Hegemonic Masculinity, Masculine Gender Role Stress, and Female Same-Sex Intimate Partner Violence}

Research over the past several decades has demonstrated that the endorsement and internalization of hegemonic masculine norms is positively associated with heterosexual men's perpetration of intimate partner and sexual violence (IPVSV) toward women (Moore \& Stuart, 2005; Murnen, Wright, \& Kaluzny, 2002; R. M. Smith et al., 2015). In particular, this line of research has demonstrated that attitudes supportive of male dominance and the subordination of women are strongly predictive of heterosexual men's perpetration of IPVSV. This is especially true among men who experience higher levels of masculine gender role stress, which is defined as the experience of stress or anxiety in gender-relevant situations that in some way challenge or upend one's identity as masculine and male (Eisler, Franchina, Moore, Honeycutt, \& Rhatigan, 2000;

Malamuth, Linz, Heavey, Barnes, \& Acker, 1995; Moore et al., 2010; Sheffield, 1987; R. M. Smith et al., 2015; Truman, Tokar, \& Fischer, 1996). A possible explanation for these relationships may be that men who adhere to hegemonic masculine norms (i.e., tough, dominant, high status, etc.) experience stress in response to perceived threats to their achieved dominant masculinity. Because violence is a central component to the prescribed roles and behaviors associated with hegemonic masculinity, perpetrating 
violence toward an intimate partner is an immediate and tangible way to re-establish a dominant and masculine status (Eisler et al., 2000; Luddy \& Thompson, 1997).

However, the internalization of a culturally defined normative standard for the dominant class is not necessarily restricted to a person's biological sex or gender identity, nor is it necessarily restricted to existing as one of two ends of a binary gender spectrum. As described above, members of systematically marginalized groups may internalize and adhere to the same cultural norms that function as tools for oppression. For instance, sexual minority women who identify with masculinity may internalized the normative beliefs associated with traditional hegemonic masculinity. Given that the most visible and stereotypic hegemonic masculine norms are associated with violence and dominance and that hegemonic masculinity is, by virtue of its dominant status in our culture, understood as a default form of gender, sexual minority women's experiences with their own masculinities may mirror this culturally established default masculinity. Sexual minority women's experiences with gender role stress, then, may elicit violent and/or manipulative behaviors toward their female intimate partners. Such behavioral responses to stress in gender-relevant situations be a function of a combination of gender role stress and experiences of sexual minority stress (e.g., stigma consciousness, internalized homophobia and misogyny). Thus, while the heteronormative theories and empirical data linking adherence to hegemonic masculinity and masculine gender role stress to heterosexual men's perpetration of IPV toward women provide a contextual framework for understanding the mechanisms underlying IPV in general, such explanatory models lack the intersectional perspective necessary to address female same-sex IPV. Thus, eight existing survey measures designed to assess various forms of gender identity, gender 
expression, hegemonic gender role adherence, and gender role stress will be evaluated in this study in terms of their accuracy in assessing the range of gender-relevant experiences among sexual minority women as well the intersections among sexual minority women's experiences with the previously described minority stress constructs and domains: (1) the Bem Sex Role Inventory (BSRI; Auster \& Ohm, 2000; Bem, 1981a), (2) the Gender Expression Measure among Sexual Minority Women (GEM-SMW; Lehavot \& Simoni, 2011), (3) the Masculine Gender Identity Scale for Females (MGISF; Blanchard \& Freund, 1983), (4) the Femininity Ideology Scale (FIS; Levant et al., 2007), (5) the Male Role Norms Inventory - Revised (MRNI-R; Levant et al., 2010), (6) the Social Dominance Orientation Scale (SDO; Sidanius \& Pratto, 2001), (7) the Feminine Gender Role Stress (FGRS; Gillespie \& Eisler, 1992), and (8) the Masculine Gender Role Stress (MGRS; Eisler \& Skidmore, 1987) The primary constructs represented in these measures and their relevance to this study are shown in Table 1.

\section{The Need for Empirical Research Specific to FSSIPV}

The study of female same-sex IPV is a difficult, but necessary, area of research to pursue given that recent reports of IPV incident and prevalence rates suggest that the prevalence of violence within same-sex couples is far greater than what previous estimates represent (Messinger, 2011; Walters et al., 2013). However, the available body of FSSIPV-related research is lacking in consistent empirical evidence necessary to inform intervention and prevention strategies at the individual, community, and policy levels (Ristock, 2011). Unlike research related to IPV within heterosexual relationships, no known research exists that specifically examines, whether theoretically or empirically, the link between minority stress, gender role adherence, and gender role stress within 
female same-sex romantic relationships and FSSIPV. As I have argued throughout this review, those who do not "fit in" with the dominant culture are often subject to insidious forms of oppressions and violence, and these oppressions are experienced across multiple levels of analysis (i.e., minority stress; DiPlacido, 1998).

Within the body of available IPV-related research, empirical evidence exists in support of the general theory that intimate partner violence is perpetrated as a reactionary behavior on the part of the perpetrator in response to stimuli outside of the intimate relationship (e.g., gender role stress, economic stress, minority stress, etc.; Balsam \& Szymanski, 2005; Eisler et al., 2000; Moore \& Stuart, 2005). This theory, however, is based almost exclusively on empirical evidence specific to IPV among heterosexual couples in which the male-identified partner is the perpetrator. Hetero-patriarchal societies have historically posited the male-identified member of a heterosexual romantic relationship as the "partner in control". Research over the past few decades has shown that the norms upholding this ideology (e.g., status, economic success, dominance, etc.) are linked to intimate partner aggression within heterosexual contexts (Johnson, 2006; Moore \& Stuart, 2005). In addition, theoretical and political perspectives related to intimate partner violence, put forth in large part by the rhetoric of Second Wave Feminism in the US, have posited women as non-violent for various reasons that were intended to further social and political movements (Burke \& Follingstad, 1999; Donovan \& Hester, 2011).

Empirical research is needed that systematically investigates whether and how the identification with masculinity and internalization of hegemonic masculine norms and beliefs influence the perpetration of FSSIPV, as well as the intersectionality of sexual 
minority women's experiences with minority stressors and the potential influence of that intersectionality on FSSIPV perpetration. Because research related to sexual minority women's experiences of gender role identity and expression is internally incongruent, we cannot determine whether models and measures of heterosexual IPV fit women's samesex relationships and their experiences of IPV (Davis \& Glass, 2011; Hassouneh \& Glass, 2008). That is, the present understanding of sexual minority women's experiences remains inconsistent in terms of how gender-relevant constructs (e.g., gender identity and expression, gender role stress) are conceptually defined and empirically measured. This inhibits the ability to accurately assess the interrelations among these constructs, which have been comparatively consistently defined and meticulously empirically examined among heterosexual people, as well as their influence on IPV perpetration and victimization among sexual minority women. Further, the presumptuous application of a heteronormative model to the experiences of sexual minority women is in support of a restrictive gender binary construction of society perpetuated to maintain the status quo. This devastating limitation reinforces the heterosexist notion that groups and individuals who do not identify with heterosexuality are essentially deviant and inferior to heterosexual people. Resisting the reductive perspective resulting from heteronormative frameworks of IPV will not only shed light on the experiences of sexual minority women, but will also elucidate nuanced ways to consider gender role adherence, gender role stress, minority stress and gendered violence in a broader context (Balsam \& Szymanski, 2005; Ristock, 2011).

The introductory quote for this review was written by a member of the Lesbian Task Force of the National Coalition Against Domestic Violence (NCADV; Hart, 1986; 
Lobel, 1986) as a part of her reflections on an organized dialogue that occurred during September of 1984 within the Coalition on how to address lesbian battering and incorporate appropriate services for lesbian IPV survivors. At the end of her reflection, Hart (1986) concludes the following:

Even if the lesbian community cannot yet figure out effective and safe ways to confront batterers and does not know how to definitively prevent lesbian assaults or how to make our community safe for battered lesbians, I trust that we will work on solutions. Meanwhile, we do know how to provide services to battered women and we have a moral and political obligation to promptly see that adequate services are available to battered lesbians in our communities. We do not need to wait or further deliberate on how to provide services to battered lesbians. This is not to provide us with an excuse for avoiding these harder issues. It is to say that our first obligation is to provide safety and advocacy for lesbians abused in intimate relationships. (p. 15)

Given that 20 years have officially passed since this dialogue occurred and that the availability of intervention services alone for sexual minority women experiencing violence in their intimate relationships remains minimal at best throughout the U.S., there is clearly a substantial amount of work to be done to address this issue. The theoretical and empirical rationale for the present research activities described below is designed from this realization of the persisting need for a comprehensive understanding of the contextual factors and underlying mechanisms that drive FSSIPV (Ristock, 2011). 


\section{Part II. The Present Study}

Although we listened to battered, non-lesbian women to understand women abuse, we are not anxious to listen to battered lesbians to understand lesbian violence. (Hart, 1986, p. 15)

This study interviewed fourteen sexual minority women recruited from the greater Portland area to identify dimensions of their experiences related to minority stress, gender identity, gender expression, hegemonic gender role adherence, and gender role stress that may be relevant to understanding women's use of violence toward their samegender intimate partners (see Figure 2). This study constitutes the first phase of inquiry situated within a larger program of research aimed at informing intervention and prevention strategies for female same-sex IPV through a multilevel and intersectional approach to social change research and community action. The primary goals of this study are to (a) inform the constructs and implied relations currently within the working intersectional model predicting female same-sex IPV (see Figure 2), and (b) to evaluate the construct validity and construct domain coverage of a subset of currently available gender, minority stress, and IPV-relevant survey measures to determine their efficacy at assessing sexual minority women's experiences (Keeley, Al-Janabi, Lorgelly, \& Coast, 2013; Messick, 1995; Vogt, King, \& King, 2004). The subset of measures to be evaluated in this study (see Table 1) are chosen because they represent the most widely used measures within the research frameworks they were developed. This project was conceptualized in favor of gaining a contextually relevant and conceptually appropriate inquiry on this social issue. 
In order to develop an empirically practical foundation for addressing female same-sex IPV, this study is designed in resistance to a comparison of sexual minority women's experiences of violence to an arbitrary default (i.e., heteronormativity) and specifically challenges prevailing dominant heteronormative research and practice frameworks. By fostering a space for amplifying the voices of this systematically marginalized population and working with a diverse group of community partners, this study is intended to serve as a foundational phase of research and community partnership building, from which subsequent planned phases of participatory action research will be developed. In sum, the current project and the larger program of research in which it is situated are aimed at building community capacity for sustainable social change and violence prevention through a careful and intersectional approach to gaining a more accurate understanding of the individual, contextual, and historical factors surrounding the continued existence of intimate partner violence, particularly among systematically marginalized populations. 


\section{Chapter 4. Methods}

\section{Research Questions}

This study is guided by the following research questions (see Table 1 and Figure

2):

- RQ1. To what extent are sexual minority women's experiences and identities accurately captured by the constructs present in the intersectional model adapted from R. M. Smith et al. (2015)?

- $\quad$ RQ1A. Are sexual minority women's experiences and identities sufficiently represented in the model constructs?

- $\quad$ RQ1B. Do the relations among the constructs in the intersectional model accurately represent sexual minority women's experiences and identities?

- $\quad$ RQ2. To what extent are sexual minority women's experiences and identities adequately captured in survey measures developed to assess the constructs in the intersectional model?

- $\quad$ RQ2A. Are the constructs in the intersectional model sufficiently covered by the survey measures?

- $\quad$ RQ2B. Are the operational definitions of the constructs in the survey measures for the intersectional model accurately representative of sexual minority women's experiences and identities?

\section{Emergent Design}

This study followed an emergent design approach in which modifications to individual design components were implemented throughout the data collection and 
analysis processes. Any modification to the original study design was based primarily on experiential and content-based knowledge gained through participant recruitment, interviews, and data analytic procedures implemented for this study (Morgan, 1996a). For instance, several minor adjustments were made to the participant recruitment processes, such as modifications to recruitment flyers and recruitment settings in order to improve response rates. The two versions of recruitment flyers are provided in Figure 5. The most substantial design modification, however, was to the mode of data collection itself. In the original study design, four-to-five focus groups would be conducted with sexual minority women recruited from the greater Portland, OR area. Each of the originally conceived focus groups consisted of a specific set of purposively-driven participation criteria (see Table 2), which were in service to maximizing the quality of data gained through each focus group. However, in the early stages of participant recruitment under this original design, it became increasingly difficult to find participants who were both eligible and available to participate in a given focus group. As such, the study design was modified to include one-on-one interviews as another way eligible individuals could participate. With this modification, ten individuals (71\%) participated in one-on-one in-depth, semistructured, open-ended interviews and four (29\%) participated in a similarly semistructured and open-ended focus group. All activities conducted for this study were approved by Portland State University's Institutional Review Board, including any modifications to the overall design or study protocols, and permissions were requested from and granted by all recruitment venues and community partners involved in recruitment and data collection prior to conducting any such activities. 


\section{Sampling, Participant Recruitment, and Community Partners}

Target population and sampling frame. The overall target population for this study consisted of women who identify as lesbian, gay, bisexual, transgender, and/or queer (i.e., sexual minority women, or "SMW"). The sub-populations of interest for this study included (1) SMW with histories of intimate partner violence perpetration and victimization, specifically in their same-gender relationships, and (2) SMW with no history of intimate partner violence (regardless of perpetrator and victim gender). The sampling frame for this study was defined in two parts corresponding to each of these sub-populations of interest. The overarching sampling frame was defined as adult sexual minority women (described above), aged 18 and over, currently residing in the greater Portland, OR area. A more specific sampling frame was also defined to include SMW who are currently receiving services from IPV-specific community-based service providers (e.g., survivor/victim advocacy and batterer intervention program services, violence prevention and education organizations, etc.). In addition to these IPV-specific groups, participants were also recruited on the basis of their current relationship status in an effort to explore potential differences in experiences among currently single SMW and women who are currently in same-gender romantic relationships. Thus, all participants were recruited according to six-dimensions related to relationship status and IPV experiences defined under this study's complete sampling frame: (1) single with no IPV history; (2) single with IPV victimization experiences; (3) single with IPV perpetration experiences; (4) in a relationship with no IPV history; (5) in a relationship with IPV victimization experiences; and (6) in a relationship with IPV perpetration experiences. The commonality across each of these six dimensions is that all individuals included in 
the sampling frame identified as lesbian, bisexual, transgender, and/or queer women. Recruitment procedures and participant characteristics for this study's final sample are provided below.

Participant recruitment and community partners. Participant recruitment and data collection occurred over the course of 14 months, during which I employed multiple strategies to obtain a sample of participants that both fit the above-described sampling frame and provided a diverse breadth of perspectives to address this study's research questions. Figure 6 provides a visual representation of the study's participant recruitment and data collection timeline. The top half of Figure 6 displays participation dates, with one-on-one interview participants represented as 'P\#' and the four focus group participants represented within the single 'Focus Group' point. The bottom half of Figure 6 displays my community-engaged recruitment efforts over the course of the fourteen months of active participant recruitment and data collection. Recruitment efforts are represented by the community partners assisting with participant recruitment. The purposively-driven community-engaged recruitment efforts displayed in Figure 6 are detailed below, followed by descriptions of the convenience sampling methods I employed alongside my purposive recruitment efforts.

Purposive sampling. Initial participant recruitment efforts began in November of 2014 upon receiving approval for the study's initial protocol from Portland State University's (PSU) Institutional Review Board. These initial recruitment efforts involved both flyer postings (see Figure 5) and face-to-face interactions with potential participants at PSU's Women's Resource Center (PSU-WRC) and Queer Resource Center (PSUQRC). With ample help from both PSU-QRC's communications staff and PSU-WRC's 
Executive Director at the time, I was able to gain a sense of the potential response rate and characteristics of individuals recruited via their affiliation with either, or both, an LGBTQ-specific organization (i.e., PSU-QRC) and an IPV survivors' support organization (i.e., PSU-WRC). While both recruitment venues yielded an encouraging number of potential participants for the study within the first couple of months of participant recruitment, scheduling a sufficient number of eligible individuals for a given focus group category soon became a barrier. As such, the study's design was modified in April, 2015, to include one-on-one interviews, which could be scheduled and conducted much sooner upon determining an individual's eligibility than was possible under the original design.

LGBTQ-specific purposive sampling. As a volunteer for Pride Northwest (Pride NW), a Portland-based LGBTQ-specific non-profit organization, I was able to distribute by hand throughout the three-day (June 13-15) 2015 Northwest LGBTQ Pride Festival in Portland, OR approximately 50 flyers advertising the study and inviting one-on-one interviews (see Figure 5). I also distributed flyers advertising the one-on-one interviews at volunteer-only events hosted by Pride NW and Q-Center, another Portland-based LGBTQ-specific non-profit organization, prior to and in the months following the annual Pride Festival. While distributing flyers at these events, I encouraged individuals I spoke with to spread the word about the study in hopes of gaining access to additional potential participants via word of mouth. Printed flyers were also posted at Q-Center in North Portland and at Portland State University's Queer Resource Center (PSU-QRC). I also provided information about the study and stacks of wallet-sized flyers and my business 
cards to the staff and volunteers at each of these three LGBTQ-specific organizations (i.e., Pride Northwest, Q-Center, and PSU-QRC).

$I P V$-specific purposive sampling. IPV-specific community partners included sexual violence and IPV-specific victims' services organizations (e.g., Portland Women's Crisis Line, Bradley Angle, Clackamas Women's Services, Portland State University's Women's Resource Center), batterers intervention programs (e.g., Allies in Change), and IPV-specific organizations and coalitions not providing direct services to survivors or offenders (e.g., the Oregon Coalition Against Domestic and Sexual Violence, the Oregon Attorney General's Sexual Assault Task Force, Men Engaging Now, and the Tri-County Batterers Intervention Providers Network). Direct-service community partners (e.g., Bradley Angle's LGBTQ IPV survivor response program, Clackamas Women's Services, Portland Women's Crisis Line, etc.) provided information about the study to clients as they deemed appropriate based on their knowledge of their programs' participants' current situations and backgrounds. In addition, all IPV-specific community partners helped with recruitment efforts by distributing electronic flyers over various relevant listservs and at their organizations.

Convenience sampling. Community partners at local higher-education institutions other than Portland State University (i.e., Reed College, Lewis and Clark College, Portland Community College - Southeast Campus) also helped with recruitment efforts by posting printed flyers on their own campuses. Printed flyers were also posted at local Portland businesses (e.g., Powell's City of Books and multiple Fred Meyer locations) and across Portland State University's (PSU) downtown Portland campus. The PSU Psychology Undergraduate Advising Office and Four Psychology professors at PSU 
further helped with recruitment by posting printed flyers to their office doors and/or distributing printed and electronic flyers to their current classes during the Spring, Summer, and Fall 2015 academic terms. Collectively, the convenience sampling methods described here and the above-described purposively-driven sampling methods yielded the 10 one-on-one interview participants for this study. Snowball sampling was used for recruiting the four focus group participants. The first two participants recruited for a focus group were acquaintances of mine, who were very interested in participating in the study and offered to help with recruiting at least two additional individuals to participate in a focus group. Because the first two participants were in a relationship and neither had any history of IPV victimization nor perpetration, I provided them with the eligibility criteria details for the second focus group category (see Table 2), which they used in determining which of their friends and acquaintances they would reach out to about the study. Using this snowball method, six individuals were recruited to participate in the focus group, with the final focus group consisting of four of those six individuals.

\section{Participants}

All participants and potential participants, regardless of purposive or convenience recruitment method, were given the same information regarding the study's purpose and possibilities for participating. That is, for each of the recruitment methods detailed above, I used the following script as a guide for providing potential participants (i.e., individuals who expressed interest in the study by contacting me via the contact information provided on the flyers provided in Figure 5) with pertinent details about the study prior to conducting the screening interviews: 
This project is being conducted by Rachel Smith under the direction of Dr. Eric Mankowski at Portland State University's Department of Psychology. We are inviting women who identify as lesbian, bisexual, transgender, or queer to participate in individual interviews for a research study that is ultimately aimed at addressing intimate partner violence in women's same-gender relationships; however, the actual interviews will not include specific questions will about violence, but will include questions about stereotypes participants have heard related to lesbian, bisexual, transgender, and queer women, and their thoughts on these stereotypes.

[FOR POTENTIAL ONE-ON-ONE PARTICIPANTS]: Each interview is expected to last approximately 1 hour, and will occur at Portland State University's campus in Downtown Portland, OR. Each participant will be given a $\$ 15$ gift-card to either Fred Meyers or Powell's Books in appreciation for their time and participation, as well as $\$ 5$ to help with transportation costs.

[FOR POTENTIAL FOCUS GROUP PARTICIPANTS]: Focus groups are expected to last approximately 2 hours. Each participant will be given a $\$ 30$ gift-card to either Fred Meyers or Powell's Books in appreciation for their time and participation, as well as $\$ 5$ to help with transportation costs.

After providing this general information about the study, I asked each potential participant if she was interested in participating. I conducted a screening interview using the questions provided in Table 3 with individuals who responded affirmatively, and thanked those individuals who responded otherwise for their time and assured them that their names and contact information would be removed from the study's contact list. All 
potential participants were asked how they learned about the study. The eligibility criteria and corresponding screening interview questions, as well as the post-interview/post-focus group survey questions, are listed in Table 3. Individuals were deemed ineligible to participate only if they responded "no" to the first screening interview question, which asked potential participants if they identified as an LGBTQ woman, or if they responded "yes" to the second screening interview question (i.e., "Are you currently in a romantic relationship?") but indicated that their current romantic partner did not identify as a woman.

The final sample of eligible participants for this study consisted of fourteen women who identify as lesbian $(n=5 ; 35.7 \%)$, gay $(n=1 ; 7.1 \%)$, bisexual $(n=4$; $28.6 \%)$, and queer $(n=3 ; 21.4 \%)$. While the recruitment, screening, interviewing, and post-survey protocols did not include specific questions about participants' cis- and transgender identities, one participant identified herself as a transwoman during the recruitment and screening process, and two participants indicated that they prefer both "she" and "they" pronouns in their post-interview survey responses. At the time of their participation, $10(71 \%)$ participants were currently in a romantic relationship, four $(29 \%)$ were single, five $(36 \%)$ had experienced victimization of intimate partner violence from a former partner, two $(14 \%)$ women had experienced victimization from and had perpetrated IPV toward a former intimate partner, one participant had perpetrated IPV toward a past female-identified partner and had no past IPV victimization and half of the participants $(n=7)$ reported having no history of IPV victimization nor perpetration. Participants ranged from 18 to 52 years of age $(M=32 ; S D=12)$. Eight $(57 \%)$ 
participants identified their race as white and six (43\%) identified as multiracial (see Table 4 for a comprehensive list of participant demographic variables).

\section{Data Collection Procedures}

Settings and logistics: One-on-one interviews. All one-on-one interviews were conducted in a small meeting space located in the Psychology Department at Portland State University. This meeting space, the Edith Sullivan Memorial Library, is semiprivate with the only entrance consisting of two glass doors facing the Psychology Department lobby, which were closed to respect participants' privacy and to reduce the amount of sound coming into the room from the outside, and a single stained-glass window facing the outside. I chose this setting for both its convenient location, as well as the relatively less sterilized, more welcoming atmosphere of the room relative to the typical meeting space available in a university setting. I provided each one-on-one participant with information about the interview location and made sure this location was convenient and accessible to them for the chosen interview time at least 24 hours prior to each interview. One-on-one interview participants were also informed that the interview was expected to last approximately one hour and that they would be compensated for their time with $\$ 15$ gift-cards to their choosing of either Powell's Books or Fred Meyer, as well as $\$ 5$ in cash to help with transportation costs. One-on-one interviews lasted an average of $M=0.76$ hours (i.e., approximately 45 minutes) each ( $S D=0.16$ hours, or 9 minutes).

Settings and logistics: Focus group. The first two participants recruited for the focus group offered to provide their home as a space to hold the focus group, which I agreed to as this setting seemed would be the most comfortable for all of the individuals 
recruited for the focus group. We also decided to organize the focus group meeting as a Sunday brunch to further facilitate a comfortable setting for the group. Thus, the full focus group meeting lasted approximately three hours; however, approximately half of that time was spent in conversation about food and everyone getting acquainted with each other or catching up after having not seen one another for some time. The actual focus group began about an hour into the brunch meeting and lasted approximately 1.5 hours. At the end of each interview, I asked each participant to complete a brief 8-item questionnaire primarily assessing demographic information, with two questions at the end of the survey asking participants (a) why they participated in the study and (b) if they would be interested in participating in a second interview or focus group. While each participant completed the post-interview/post-focus group survey, I placed the gift-card and $\$ 5$ cash compensation in a card, along with a wallet-sized contact card containing the names of IPV-specific organizations in the Portland area (e.g., Bradley Angle's LGBTQ Program, Portland Women's Crisis Line, etc.), a couple of wallet-sized flyers, and one of my business cards. I also hand-wrote a short "Thank you" note on each card containing these materials and explained each item to each participant once she was finished with the post-interview/focus group survey.

Interview and focus group content. This study was designed as primarily exploratory in its intent, with specific attention given to participants' perspectives regarding the influence of gender role adherence, gender role stress, and minority stress on the lives of sexual minority women. The moderating guides (see Appendices $A$ and B) were both designed to allow for an exploration of how gender stereotypes typically ascribed to sexual minority women impact their day-to-day experiences of minority 
stress, their same-sex romantic relationships, and their potential experiences of FSSIPV victimization and perpetration. In order to do this, the one-on-one interviews and the focus group conducted for this study were intentionally semi-structured in design with questions that guided participants to consider their experiences with gender-relevant topics while allowing the conversations to flow according to the participants' perspectives on the topics, rather than being led step-by-step through each of the constructs of interest for this study (see Figure 7).

While primarily open-ended, both the interview and focus group guides specifically attend to participants' perspectives regarding the influence of gender role adherence, gender role stress, and minority stress on sexual minority women's identities and day-to-day lives. In addition, the one-on-one interviews included a set of questions relating to participants' definitions of their identities in general. For this portion of the one-on-one interviews, participants were first asked to explain what the general concept of identity meant to them and then asked to list five words that best described their identities (see One-on-One Interview Guide in Appendix B). Collectively, the one-on-one interviews and the focus group served to inform the construct definitions within the working intersectional model, as well as to determine the appropriateness and efficacy of survey measures relevant to the construct domains present in the intersectional model in terms of accurately reflecting the range of potential experiences and identities among sexual minority women (Morgan, 1996a). The construct domains assessed by survey measures specifically of interest in this study include (see Table 1): gender identity and expression (i.e., the Bem Sex Role Inventory and the Gender Expression Measure among Sexual Minority Women; Bem, 1981a; Lehavot, King, \& Simoni, 2011), traditional 
gender role adherence (i.e., the Male Role Norms Inventory and the Femininity Ideology Scale; Levant et al., 2010, 2007a), gender role stress (i.e., the Masculine and Feminine Gender Role Stress Scales; Eisler \& Skidmore, 1987; Gillespie \& Eisler, 1992), and sexual minority stress (Mohr \& Fassinger, 2000; Pinel, 1999; i.e., the Lesbian Internalized Homophobia Scale, the Outness Inventory, and the Stigma Consciousness Questionnaire; Szymanski \& Chung, 2008; Wagner, 1998). 


\section{Chapter 5. Data Analysis}

Inductive contacts with respondents can supplement deductive contacts with theory as a source of hypotheses. (Morgan, 1996b, p. 27)

\section{Research Question One (RQ1): Inductive Thematic Analyses}

Analyses of the qualitative narratives were guided on principles of Grounded Theory (GT) and inductive thematic analysis (Braun \& Clarke, 2006; Charmaz, 2011; Fereday \& Muir-Cochrane, 2006). Data obtained from the one-on-one interviews and the focus group were first analyzed using an inductive (i.e., bottom-up) approach (Charmaz, 2006; Creswell, 2012; Strauss \& Corbin, 1990). In order to further familiarize myself with the data, I transcribed each interview and the focus group interview, instead of seeking a third party to complete the transcription. Following the general approach of GT, I also used the time spent transcribing each interview for memoing notes about the content of the data, as well as reflections on the interviewing methods employed throughout this study. The transcription and memoing processes thus informed both my initial analytic impressions of the data, as well as my methodology moving forward. That is, because I began transcribing the interviews while continuing participant recruitment for one-on-one interviews, the analytic memos I made while transcribing the interviews informed interviewing strategies implemented in later interviews conducted for this study, as well as my determination for when enough interview data had been collected to address the two previously outlined research questions. Following the completion of data collection, transcription, and initial analytic memoing, I employed a systematic opencoding analysis to generate a comprehensive code list reflecting the content of the data (Charmaz, 2011; Creswell, 2012; Onwuegbuzie, Dickinson, Leech, \& Zoran, 2009). 
Using an inductive thematic analysis approach, I reduced the initial code list generated through my open-coding analysis by focusing the code list on two points: (1) the constructs of interest for this study based on the working intersectional model depicted in Figure 2, and (2) the themes most salient or meaningful across the one-on-one interviews and focus group data. Details for determining the saliency and meaningfulness of themes is further detailed below.

The focused-coding analysis resulted in a final codebook consisting of 103 thematic codes categorized according to the constructs of interest. In a third inductive analytic phase, I developed definitions for the final codebook by examining relations among the codes contained in the final codebook based on the aggregated content of interview and focus group data segments to which each code was applied. In sum, the above-outlined systematic inductive thematic analysis approach guided by GT consisted of three consecutive analytic phases, each of which is detailed below.

First-phase inductive thematic analysis: Open-coding. My original data analytic plan began with segmenting focus group transcripts according to each speaking turn and then coding each segment for meaningful content. However, once data collection was complete and I began the process of cleaning and organizing the data, it became clear that this portion of the analytic process needed to be adapted to more appropriately suit the structure and content of the data. That is, the group dynamic experienced during the one focus group conducted for this study was much less structured and far more conversational than I had originally anticipated in the planning phase of this project. As a result, segmenting the content of the focus group's transcript according to each speaking turn would have resulted in a loss or distortion of the participants' meanings in their 
responses to interview questions or prompts. Further, the original study design was modified to include one-on-one interviews to replace planned focus groups for which an inadequate number of participants were recruited (see Methods section for more details on this modification). This methodological adaptation resulted in one-on-one interviews comprising the majority of data collected for this study, and the originally planned initial segmenting procedure would not have been appropriate for the structure of the one-onone interview data. As such, all interview transcripts for this study, including the focus group, were instead initially segmented according to the four overarching constructs reflected in the focus group and one-on-one interview questions: (1) Stereotypes (i.e., the introductory activity) and the influence of stereotypes on LGBTQ women's experiences and identities, (2) Identity, gender identity, and gender expression, (3) Gender identity, gender expression, and gender role adherence, (4) Gender role stress and sexual minority stress.

This initial segmenting process was done using a computer assisted qualitative data analysis (CAQDAS) process using the $R Q D A$ (Huang, 2014) package developed for use with $R$ statistical computing software (R Core Team, 2016). Two of the most common and useful features among the various available CAQDAS software programs, including $R Q D A$, are (1) streamlined data organization functions and processes, and (2) the ability to retrieve and combine segments of data that have been marked with a given code while maintaining linkages between coded segments and the documents from which they originated (Lewins \& Silver, 2009). Taking advantage of the combination of both of these features in $R Q D A$, I combined all responses to each of the interview and focus 
group questions within each category while maintaining each response's connection with the participant to whom it belonged.

Following this segmenting procedure, I employed an initial open-coding analysis process for the combined data for each of the above-listed four overarching construct categories were then open-coded to identify salient points among each participant's interview responses in each category. For the open-coding analysis, I created two-column tables in Microsoft Word consisting of the combined interview content, with subsections separating each participant, for each of the above-listed construct categories (Column 1), and space for adding open-code labels and notes corresponding to the interview data (Column 2). The interview content in each of these tables was separated into subsections for each participant, and then further separated by row according to each speaking turn in a given interview. After completing the open-coding analysis for each construct category, I entered all of the coding labels generated through this process in $R Q D A$ as codes and applied those codes to the appropriate content in each participants' interview transcript.

This analytic phase was guided by GT analytic principles and served as my first step in getting to know the interview data at a more granular analytic level. Following the general approach prescribed under GT methodologies, I was intentionally speedy in the manner I developed each code during the open-coding process in order to reduce, to the best of my ability, the imposition of preexisting theoretical concepts and terms upon the data (Charmaz, 2006). That is, my initial coding process involved developing a list of codes, or indexes, that represented close summaries of the participants' points throughout the interviews by primarily using each participant's own words in constructing a given code, rather than reconstructing the participants' points in more theoretically-based terms. 
Doing so allowed my analysis moving forward to remain grounded in the experiences of this study's participants as reported by the participants, rather than being based solely in the theoretical foundation of this study.

The code list resulting from the initial open-coding analytic phase described here consisted of 2,318 codes. Because these codes were developed to represent the data as closely as possible, this code list ultimately served as a list of indexes throughout the analysis phases described below. Following the completion of open-coding a given category, I reviewed the resulting list of 2,318 indexes (i.e., codes/code labels) and reexamined any code labels that seemed more of a reflection of my own theoretical mindset than the participants' actual words. After cross-checking any such instances with the original data from which I developed a code label, I used a bracketing scheme to separate my own theoretical code labels from the labels derived directly from participants' own words (e.g., "[INTERSECTIONALITY] Ppl more comfortable talking about my sexuality than my race"). This bracketing scheme facilitated my second phase of qualitative analysis described below. An illustration of the open-coding process described here is available in the example excerpt of open-coded interview data presented in Table 5.

Second-phase inductive thematic analysis: Focused-coding. Following the completion of all open-coding, I conducted a second coding phase aimed at reducing the 2,318 indexes/codes created through the initial open-coding analysis. (i.e., "focusedcoding"; Charmaz, 2006; Saldaña, 2015). Guided by GT, I used the indexes developed through my initial analysis to determine those themes. In other words, my second-phase of analysis was more along the lines of what I like to call "code-coding", and involved me assigning labels, typically theory-driven, to each the open-coding indexes. This 
"code-coding" process yielded a list of 103 codes occurring within each of the four construct categories of interest for this study: (1) The influence of stereotypes related to LGBTQ women, (2) Identity, gender identity, and gender expression, (3) Gender roles and gender role adherence, (4) Gender role stress and sexual minority stress.

I cross-examined the four code lists to determine were unique to a given construct category's code list and which codes were common among two, three, and four code lists. Based on this focused hierarchical analysis, the code list was reorganized into the following higher-level construct categories: (1) Identity, (2) Gender, (3) Minority Stress, and (4) Intersectionality. Codes that occurred within the original first construct category (i.e., The influence of stereotypes related to $L G B T Q$ women) were folded into the Minority Stress category, and codes within the fourth category are those that did not distinctly occur within one of the first three construct categories. Specifically, codes categorized under "Intersectionality" occurred in one of the following construct category combinations representing the three sub-categories under "Intersectionality": (a) Minority Stress and Identity, (b) Minority Stress and Gender, or (c) Minority Stress, Identity, and Gender. The codes contained within the final codebook were then examined according to their relations among one another, both within and between each code's respective category or categories.

Third-phase inductive thematic analysis: Axial coding. As a third and final analytic phase I conducted an axial coding analysis of the above-described code list in which I examined an uncategorized version of the code list resulting from my focusedcoding and hierarchical coding analyses in conjunction with the original open-coding indexes and interview data segments to which each code in the full code list referred. For 
the final phase in my inductive qualitative analytic approach, I constructed a data table consisting of five columns: (1) Code-name, (2) Open-coding Label, (3) Construct category, (4) Interview/Focus group data segment, and (5) Interview case number ("PID").

The above-listed three major category domains and corresponding sub-categories identified in the second analytic phase described above were then defined according to the combination of (a) theoretically-based conceptualizations and definitions relevant to each category and sub category, (b) each code's meaning according to the interview data from which it was derived, and (c) the interrelations among the categories, subcategories, and codes in the code list. The full hierarchically-organized codebook with definitions is presented in Appendix $C$.

I conducted the systematic inductive qualitative analytic approach described above to address RQ1: To what extent are sexual minority women's experiences and identities accurately captured by the constructs present in the intersectional model adapted from R. M. Smith et al. (2015)? Specifically, I sought to address RQ1a (Are sexual minority women's experiences and identities sufficiently represented in the model constructs?) through the first and second inductive analytic phases, while the third and final phase of analysis served to enhance the first two in addressing RQ1b: Do the relations among the constructs in the working intersectional model accurately represent sexual minority women's experiences and identities? Findings from this inductive thematic analysis guided my below-described analyses to evaluate the survey measures of interest for this study, which is described below. 


\section{Research Question Two (RQ2): Evaluation of Existing Survey Measures}

In a second set of qualitative analyses, I used the final codebook and constructrelevant findings from the above-described systematic inductive analytic approach to address $R Q 2$ (i.e., To what extent are sexual minority women's experiences and identities adequately captured in survey measures developed to assess the constructs in the intersectional model?). Specifically, I applied the inductively-derived codebook to the survey measures listed in Table 1 to evaluate each measure in terms of (1) the content coverage of the measure as a whole, and (2) the appropriateness and accuracy of the survey's individual items according to the constructs named and defined in the final codebook (see Appendix C).

Prior to completing the codebook application to the measures listed in Table 1, I made the decision to remove the Masculine Gender Identity Scale for Females (MGISF; Blanchard \& Freund, 1983), as well as the Revised Conflict Tactics Scales (CTS-2; both Perpetration and Victimization; Straus, 1979; Straus, Hamby, BoneyMcCoy, \& Sugarman, 1996) from the list of measures to be evaluated in the current study. The MGISF was removed due to its structural incompatibility with the scope of this study. Specifically, the MGISF is designed as a clinical measure with each item containing its own set of response options, each with varying clinical weights, rather than a single rating scale applicable to all items in the measure, as is the structure of all other measures evaluated in this study. The CTS-2 victimization and perpetration measures were also removed from the measurement evaluation analysis due to this study's limited amount of qualitative data specific to IPV perpetration and victimization (CTS-2-P and CTS-2-V, respectively) to inform a meaningful evaluation of the measures. This limitation is due in 
part to the fact that no IPV-specific questions were asked in the interviews nor the focus group, as well as to the smaller than anticipated sample size for the categories of sexual minority women with histories of female same-sex IPV victimization and/or perpetration.

Codebook application to evaluated measures. The inductively-derived codebook was separately applied by myself and a trained research assistant. Before engaging in this analysis, the research assistant spent two weeks listening to and reading through each interview recording and transcript while also making analytic memos in order to familiarize herself with the data. The research assistant went through this process while I separately conducted the inductive thematic analysis process to develop the codebook that would be applied to the measures. This process was done to reduce the imposition of my own bias on the research assistant's interpretations of the data, the measures to be evaluated, and the codebook applied to the measures while also ensuring that the research assistant was well-equipped to evaluate the measures according to the inductively-derived codebook. Once the research assistant completed her review of the transcripts, codebook, and measures, she and I separately applied the codebook to each measure by assigning a maximum of two codes to each item in each measure. After familiarizing the research assistant with the codebook and the measures by applying the codebook to ten survey items randomly selected from a subset of two gender-specific measures and one minority stress-specific measure, I asked the research assistant to apply the codebook to each measure using a coding table containing three columns for each measure: (1) Item, (2) Code(s), and (3) Notes. I asked the research assistant to make any notes about her coding process, the codebook, and the measures themselves in the third "Notes" column. Once the research assistant completed her application of the codebook 
to the measures, she and I discussed our general processes and experiences with applying the codebook to the measures. Next, the research assistant and I conducted a second round of applying the codebook to the measures using a more structured approach. That is, the measures were evaluated in the second round of codebook application by going through each item in a given measure and looking for a maximum of two codes from the codebook that could conceptually replace a given item, and items to which the coder deemed no codes could be applied were coded as " $\theta$ ".

Inter-rater reliability. Once the research assistant and I completed this second round of applying the codebook to the measures, I calculated Cohen's Kappa ( $K$; Cohen, 1968) to assess the consistency among mine and the research assistant's applications of the codebook to each of the measures. To do so, I constructed dichotomous $103 \times 2$ matrices for each measure with two columns in each matrix representing mine and the research assistant's decisions to apply a given code to the measure $(\mathbf{1}=$ Code Applied; $\boldsymbol{0}$ $=$ Code Not Applied). Using the two coders' columns, I calculated a Kappa statistic to assess the level of agreement across both coders for each measure. Results from this analysis of inter-rater agreement yielded a range in Kappas of $0.39-0.69$, and analysis of the overall agreement across all measures yielded $K_{\text {TOTAL }}=0.54$ (see Tables 6 and 7). The primary purpose of calculating Kappa as an indicator of interrater reliability in this study was to assess (a) the two coders' readiness to evaluate the measures using the inductivelyderived codebook (i.e., to assess the second coder's and my shared understandings of the codebook's content), and (b) to evaluate the utility of the inductively-derived codebook as a tool for evaluating a set of pre-existing survey measures. This purpose does not include establishing the highest possible level of interrater reliability as the end result of the 
analyses conducted to apply the codebook to the set of evaluated measures. Rather, in line with the exploratory nature of this study's goals, research questions, and overall emergent design, this study's inter-rater reliability analysis served to provide insight into both the level of consistency with which the codebook was applied in evaluating the measures, as well as possible ways the codebook can be further improved for use in future measurement evaluation efforts with less exploratory purposes. While there is no established nor entirely agreed upon convention for determining a suitable Kappa value, most research purposes warrant seeking a Kappa statistic of at least $K=0.41$ to indicate a moderate or better level of agreement among raters after accounting for chance agreement (Banerjee, Capozzoli, McSweeney, \& Sinha, 1999; Landis \& Koch, 1977; McHugh, 2012; Multon, 2010; Sim \& Wright, 2005; Viera, Garrett, \& others, 2005). Given this context, I accepted the achieved level of agreement across all measures (i.e., $\left.K_{\text {TOTAL }}=0.54\right)$ as suitable for this study's purposes. 


\section{Chapter 6. Findings}

The patterns of oppression found when considering these constructs contribute to the overall context of behavior. As we now view intersectionality, these individual variables are not simply additive, but intersect with one another continuously. (C. Smith, 2011, p. 143)

The collective result of the previously described three inductive thematic analysis phases is a theoretically driven and comprehensive codebook that is grounded in the experiences and identities of participants. Upon examining the relations existing within the codebook, it became clear that the constructs within the working intersectional model were reflected in participants' experiences and identities. However, my inductive analysis of the data led me to refine the definitions of the major constructs present within the working intersectional model (i.e., minority stress, gender role adherence, and gender role stress) in order to more accurately reflect the identities and experiences of sexual minority women as described by participants.

These inductively-based findings were subsequently used in my evaluation of the survey measures of interest for this study. Findings from the application of the codebook developed from the inductive analyses indicate that, collectively, the content within the evaluated measures does not sufficiently, nor always accurately, reflect the experiences and identities of this study's sample of sexual minority women. Details for how I arrived at this conclusion, beginning with findings from the first set of inductive analyses, are provided in the sections below. 


\section{RQ1 Findings: Intersectional Model Construct Definitions}

While I did not find evidence suggesting deficiencies specific to the constructs present in the working intersectional model depicted in Figure 2, my inductive thematic analyses led to the conclusion that modifications to the model are warranted in terms of the model's gender role adherence, gender role stress, and minority stress construct definitions and operationalizations. In particular, the theoretically-based operational definitions of these constructs in the model did not sufficiently reflect the collective experiences and identities shared by participants. These constructs were thus re-defined to more comprehensively and accurately reflect the experiences and identities of the sexual minority women who participated in this study. The findings and conclusions made regarding these constructs' definitions have implications regarding the intersection of the model's overarching gender and minority stress construct domains represented by the gender role stress and minority stress constructs' interaction term in the center of Figure 2. These findings addressing RQ1 are detailed below, followed by the implications of these findings for the survey measures evaluated to address RQ2.

Minority stress construct definition. Minority stress experienced by sexual and gender minority individuals and groups is traditionally operationally defined as physical, mental, and/or emotional experiences of anxiety, strain, and/or tension due to experiences of discrimination and prejudice based on one's sexual orientation (I. H. Meyer, 2003, 2015). The minority stress construct within the intersectional model was originally conceptualized according to this traditional definition. However, at each phase of analysis I became increasingly convinced that a modification of the definition was needed to fit the participants' unique and converging data. A consensus existed across interview 
participants regarding the range of minority stressors that LGBTQ women could face, how those stressors manifest in their lives, and how minority stress impacts their own identities. Specifically, minority stress experiences shared by participants were thematically tied to experiences of having ones' identity, and the expressions of that identity, defined and enforced by others (i.e., "identity policing"). The complexity underlying and resulting as a consequence from identity policing is described below, along with additional details regarding the analysis of and conclusions about the minority stress theme and construct definition.

Minority stress construct analysis and definition development. Throughout the second and third inductive thematic analytic processes (i.e., focused and axial coding), I was intrigued by Participant-7's (P7), a one-on-one interview participant, response to a question about how she felt her gender identity and gender expression(s) had been positive influences on her life, to which she responded with a description of the difficulties she faced from feeling pressured to perform a very specific role:

P7: I think that probably, um... [BRIEF PAUSE] pressure that I got to perform in a certain way from other people [emphas is added] is what led me to decide that I didn't want that, 'cause I think that without that pressure I would've just kept kind of like existing and not really figuring out um who I was or wasn't, which isn't necessarily a bad thing, um it just means that I think somewhere along in that process where you're performing for other people, eventually you'll start to perform for yourself. Um and so kind of nearing that never-ending process, like kind of the middle road of that, I guess, um has been a positive in some ways, even 
though getting there was difficult, but I kind of like what the result is looking like [emphasis added].

While the participant was asked a positively-framed question about her experiences, it seemed difficult to disentangle the positive aspects of defining and becoming comfortable with her own identity, and her expressions of that identity, from the external pressures she faced regarding who she was expected to be and how she was expected to act and look. I found this segment as illustrative of an emerging theme among participants' experiences with minority stressors and their identity development. While the identity-development processes Participant-7 describes here are not necessarily unique to sexual minority women, her experiential description of that process highlights, in my analysis, the burdens she faced as a non-heterosexual woman. She describes feeling pressured and experiencing confusion throughout her life, and at times internalizing others' expectations, until eventually coming into her own identity and expression(s) on her terms: "I think somewhere along in that process where you're performing for other people, eventually you'll start to perform for yourself" (Participant7). This is a particularly consistent thematic pattern evident throughout the one-on-one interviews and focus group data. The collective descriptions participants gave regarding internalizing others' expectations of their identities emphasized the unique complexities they experienced in understanding, developing, and expressing their identities as sexual minority women.

For instance, the following segment occurred during the focus group's (FG) conversation around identity and acceptance: 
FG-P1: ... I didn't wanna answer questions, so I allowed myself to suffer, because I didn't wanna deal with the potential of questions. Which [BRIEF PAUSE] aches.

FG-P2: And that's elements that straight people... [BACKGROUND NOISE ... FG-P1: say what you were saying, it's the elements that straight people?] don't have to think about. [BACKGROUND NOISE] So I think it's an extra step that umm it's a different step than uhh straight people would probably take. Ummm I think we all have the same basic lessons in life, and stressors come at us umm [BRIEF PAUSE] in a different way, but we all have the same lessons to learn. [FG-P4: Hmmm] So if you look at the counterpart in a straight couple, then they have other things to look at in their lives. Umm, family stressors come at 'em differently, you know, so it- I don't think it's unique to being gay or being identified as um [FG-P4: right], but I don't think there is a deeper soul searching than someone going through what you're going through. I don't go through what you're going through, I don't go through what you're going through [POINTS TO INDIVIDUALS AROUND THE TABLE], umm because my process is different [MULTIPLE: mhmm (AFFIRMATIVE)]. Do I go through the same searching? Probably, at a different level. But at the core, we all have those questions [MULTIPLE: yeah... mhmm (AFFIRMATIVE)], "how do I belong? how do I make this work? How do I go through this?" and um it's just sad that, at times, it's created this sub-culture. It's the same questions throughout, but it just created a sub-culture, and that's what we're facing. [FG-P4: That's a really good way of looking at it] It's that little pocket of people now that umm [BRIEF PAUSE] 
needed uhhh more acceptance, a little bit more courage, a little bit more- little less you know, "ohhh, I'm only going to behave this way, because I get validated that way". [FG-P1: yeah] So, we all I think at the bottom, at the baseline, we all face the same "do I belong here? am I being loved? am I going to be accepted?" but then, some of us face bigger lessons um in a big way, you know. And so those stressors are weighing in their lives differently than in my life.

In the conversation quoted above, the participants recall experiences of feeling pressured, confused, and of internalizing others' expectations. Simultaneously, however, the conversation's progression also illustrated in my analysis the complex experience of not only worrying about and/or internalizing others' expectation of your identity, but also the experience of those external expectations being magnified for individuals with marginalized identities (e.g., sexual minority women). Importantly, I did not fully recognize this emerging thematic pattern around others' expectations and minority stressors until I began the final phase of my inductive coding process and began to see an emerging thematic pattern reflecting a subtle, but impactful, layer of complexity associated with a marginalized identity that is encompassed within a community of marginalized identities. The emerging theme among participants' stories in this regard suggests that the experience of internalizing others' expectations of one's identity, and expressions of identity, can become particularly complex when those external expectations are not only imposed from others who do not share that specific identity, but also when that identity is defined, by others, in association with similar, yet distinct, identities. For example, Participant-14 (P14) identifies as a bisexual woman, an identity included in the overarching identity category of sexual minority women. However, her 
identification as a bisexual woman is in some ways defined for her both by others whose identities do not fall under "sexual minority woman", as well as by other sexual minority women. The former is apparent in the content toward the end of my one-on-one interview with Participant-14 when I decided to follow-up with her on a point she alluded to earlier in the interview:

I: Oh, I'm sorry! I had one question to clarify something [P14: Yeah!] about- you said your parents don't, and you don't- you can choose not to answer this of course [P14: Yeah, yeah, no, I'm pretty open], but you said your parents don't accept you for your bisexual identity, would they be more accepting if it was lesbian?

P14: I think so. Um...[I: Okay. Can you tell me more about that?]

P14: M- Yeah! Um my mom uhh [BRIEF PAUSE] she- she did say, when I came out to her, she said "you're either like [BRIEF PAUSE] gay or you're not, and I would've known if you were a lesbian by the time you were- like when you were a child". [BRIEF PAUSE] Yeah! And so [LAUGHS], clearly like combating that [BRIEF PAUSE] is interesting, because y-you just kinda have to be like "I don't know, mom, I just am!", you know? "and like you have to be cool with that". And um, yeah, I think if I was like "hey, I'm a lesbian", she would've been like "oh, women studies, that makes sense" [BRIEF PAUSE] yeah.

In the above-quoted content, Participant-14's experience with her mother's response to her bisexual identity constituted, in my analysis, a form of external identity definition, whereby one or more aspects of an individual's identity is defined and understood according to preconceived notions about both the individual and the focal identity characteristic(s). While the content above represents external identity definition 
experienced from someone who does not share the broader identity categorization of "sexual minority", the following excerpt from earlier in Participant-14's interview illustrates similar identity-related experiences from other sexual minority women:

I: Yeah I- I think I hear you on a lot of these things and I think it's- it's interesting because there's a lot of pub- lic- there's a lot of experience of being out in public and [P14: Yeah] particularly around the queerness part of your identity, right? [P14: Yeah] And so I'm curious though about the other way around, if you've experienced, within the com- munity, stereotyping?

P14: Yeah. Oh, absolutely. Yeah, ooh yeah. [BOTH LAUGH] Um especially as being a bisexual woman in a queer space I've never, in a s- in like a hetero space and a queer space I've never really felt like I belong to one or the other. And so um [BRIEF PAUSE] in a queer space, I'm not really queer enough. And I don't feel included in like um different move- ments or like the QRC, for example, love it, think it's a great place, but I still don't feel like it's a place for me. Um [BRIEF PAUSE] mainly just not being queer enough and being ostracized or [BRIEF PAUSE] not feeling comfortable hanging out with like a group of lesbians because I'm not lesbian, um, [BRIEF PAUSE] yeah, definitely. I don't- I wouldn't say I feel physically unsafe, like I do in a hetero community, and that's what the difference is, and so, even though it's not a space for me in my head, um it's a lot more of a sp- a safe space.

This participant's descriptions of her coming out experiences and her experiences within the LGBTQ community began to resonate as an emerging thematic pattern regarding minority stress experiences and identity development, definition, and 
expression. In particular, I suspected an emerging theme of minority stress experiences occurring in terms of the relative impact(s) of having one's identity defined and/or enforced by others. Focusing my analysis around this theme, I examined whether the emerging pattern differentiated between participants' experiences and the previouslydescribed definition of minority stress (i.e., physical, mental, and/or emotional experiences of anxiety, strain, and/or tension due to experiences of discrimination and prejudice based on one's sexual orientation; I. H. Meyer, 2003, 2015). This examination led me to develop and refine a definition of minority stress that could more accurately capture the identities and experiences of this study's sample of sexual minority women. The analytic process and findings leading to this conclusion are further detailed below.

To assess the extent to which participants' experiences with minority stress were sufficiently and accurately represented in the intersectional model (see RQ1a), I revisited the interview data segments coded with any minority stress sub-category codes in my inductive thematic analysis process (see RQ1 Analyses, and Appendix C). This allowed me to more specifically evaluate the nature of participants' minority stress experiences, as well as whether and how those experiences intersect with participants' experiences in terms of other constructs present within the intersectional model (see $R Q 1 b$ ). That is, evaluating the minority stress coding segments also involved investigating the presence or absence of non-minority stress codes in these segments, as well as the corresponding qualitative contexts in which participants' minority stress experiences do or do not intersect with other types of experiences (i.e., experiences represented by codes in the "Identity" or "Gender" codebook categories, see Appendix C). In doing so, I found that participants tended to describe their identities in ways that spoke to either identities that 
are layered or identities that are faceted. Regarding the former, participants who described their identities in terms of layers seemed to conceptualize their own identities based on a hierarchy of qualities or characteristics (i.e., certain qualities were prioritized over others in terms of what most defined their identity). This is distinct from descriptions of identity that focus around the various facets of an individuals' identity. Participants who most consistently defined and discussed their own identities in this latter form seemed to be more concerned with naming the full constellation of what makes up their identities than with any hierarchy that may or may not exist within that constellation. This distinction in how participants described their identities was most apparent in the one-on-one interviews when I asked participants to list five words to describe their identities and then asked them to describe how they came up with their lists (see Question 2 in the One-on-One Interview Guide in Appendix B). Participant-5's responses to these questions illustrate a hierarchical conceptualization of identity: I: I'm curious about how would you, if you were to choose five words to describe your identity in total... [P: Does it have to be a sentence or...?] No, just five adjectives, maybe [P: okay] that you could choose to [P: My identity, umm...] describe your identity, yeah.

P5: [BRIEF PAUSE] Ummm... [PAUSE] Hm. Black, multiracial, lesbian, spiritual, uhmmm writer.

I: [PAUSE] Nice. How easy was- or difficult, was that to come up with? P5: Um, actually pretty easy. [PAUSE] I just went in order of how do I identify myself.

I: How did you come up with that order? 
P5: Uhhh... well [BRIEF PAUSE] my black identity is gonna always be first, um 'cause I identify on the census as being black, but I'm also multiracial because my mom is half-black half-Native American and my dad's white, so that's another layer to my identity, and then being a lesbian, then I'm spiritual, and then I'm a writer, so.

Here, Participant-5 describes her identity as well as her own identity in terms of ordered layers, such as "I just went in order of how do I identify myself" and her use of "first" and "then" in the way she describes the "layers" of her identity. I then asked if she deliberately omitted certain words from her list, to which she responded by explaining the influence of her brother's mental illness on her identity:

I: Was there anything that you may have, or any words that you may have kind of skipped over in your head or like thought of in your head, but then said "no", anything like that?

P5: Uhh, 'sister'. My brother's my best friend and I've been taking care of him all my life. He has a mental illness, so it's [BRIEF PAUSE] he needs a lotta handholding [CHUCKLES].

I: And was it difficult to not include that in the five?

P5: Uhh... not really difficult, I think it's more of a personal [BRIEF PAUSE] thing, a personal connotation. [I: Yeah, it doesn't describe your identity as an individual, is that...?] Um, it does, because most of my life has spent worrying about my brother, taking care of my brother, [BRIEF PAUSE] you know, sacrificing for my brother [I: yeah], 'cause he took a lot of energy, um... [BRIEF 
PAUSE ... I: Yeah]. But it's also something I usually [BRIEF PAUSE] until you get to know me, you're not gonna really know about that.

I interpreted this latter segment as a complementary illustration of how Participant-5's definition of her identity is constructed and expressed in a deliberate manner that seemed less focused on providing an accurate and complete description of her identity and more focused on which aspects of her identity to prioritize in how she describes her identity to others. In addition, I found this participant's explanation for not including "sister" in her list as something that was "more of a personal connotation" curious in that it challenged one of my own assumptions about identity: that an individual's identity is an inherently personal concept. In examining other participants' responses to the identity-related interview questions, I found a thematic pattern emerging in which participants who defined their identities in terms of ordered layers were often the same participants whose experiences of gender and sexual minority stressors were coupled with experiences of minority stressors related to their racial and ethnic identities. Importantly, content comprising this thematic pattern highlights, in my analysis, the interconnectivity of experiences related to gender, sexual orientation, race, and ethnicity. This inter-connectivity among seemingly distinct identity domains emerged as a key component of minority stress experiences expressed by participants in that experiences of minority stressors related to multiple identity domains seemed to shape how individuals both defined and expressed their own identities. For example, Participant- 8 describes the impact of historical oppressions, as well as other forms of minority stress she has personally faced, on her experience and identity: 
P8: [SIGHS] Yeah. Um, I think that [BRIEF PAUSE] in so far as religion, um, it's just made me feel not very connected to my religious side, because um I was raised as like a Sephardic Jew, but we went to a um a Hasidic community, 'cause that was what was like around us, and so it's very difficult to have conversations about orientation, sexuality, or gender in general, um and so for them often how it is in the queer community is it's okay if you're like within some sort of binary, or if you pick one side, but you can't pick both, you can't pick everything. Um and then for race, I mean, um my mother's family who is like the black part of her family, they don't really talk about this kind of thing, so it's like if it is something that you are, unless you're like a gay man it's not really something that you're allowed to talk about, and if people know about [it], you just have to keep it on the low-low. I guess. And people won't like talk to you up-front about it, so [I figure] about somebody else. Um and then my- for my paternal side, um, they're white and they- they don't real- they- they also don't really talk about anything, um, but for them when they're confronted with it they're just like "mmm pretty sure you're just straight", and they just like talking about like boys and s- yeah. Um. But I think that probably [BRIEF PAUSE] my uh the indigenous part of my family was the one that more positively impacted the identities that I hold, because it was with that that I was able to like develop my like two-spirit woman identity, um and a lot of people don't really understand that, or like what it means, so [BRIEF PAUSE] within the queer community there's a lot of 'oh, so if your like two-spirit then that means that you're trans right?', and so it's just kind of the um like sure, yes, in technicalities of how language works, but also like within 
certain ethnic groups it's not about being like a cis person or a trans person, it's about like who you are on like a spiritual level, and that's not really something that translates very well across cultures I've discovered.

In contrast to the layered and hierarchical identity descriptions above, Participant9's responses in this portion of her one-on-one interview consistently reflected, in my analysis, a more faceted and less hierarchical conceptualization of identity. Prior to asking this participant to list five words to describe her identity, I asked her to explain how she defines the concept of identity in general:

I: Mhmm... So what do you think of when I say 'your identity'?

P9: My identity... um [BRIEF PAUSE] I don't know, I think about my personality, the traits that I give to me. I think about my sexuality, which is something that, with the same friend I've had a conversation, and she was talking about how she didn't really understand why it's such a big- [BRIEF PAUSE] a big deal for people to be like so out and proud and loud about who they are, and I was just kinda like "well, it's because people have had to be in the closet for so long that, now that we can be out, it's just kind of something that we form a community around, and something that kind of encompasses a greater part of ourselves than it might ha- than it might in a perfect world". [BRIEF PAUSE] And so I think about that, aaand... [SIGHS] I don't know, what I wanna do in the future, um how I interact with my friends and... [BRIEF PAUSE] just kind of [SIGHS] how I deal with things, and different situations.

Participant-9's definition of what the general concept of identity means to her stood out to me in contrast to identity descriptions similar to, and including, those quoted 
above in that there was a certain versatility or flexibility in Participant-9's definition of identity that was not reflected, or was less prominent, in the more hierarchical descriptions of identity from other participants. This contrast remained apparent in my analysis of how Participant-9 defined and described her own identity:

I: ... if you had to choose five words that best describe your identity, what would those five words be?

P9: Okay, um [CHUCKLES ... LONG PAUSE] I don't know, this is hard, it's like when people try to ask you what your five favorite songs of all time are. [I: I know ... BOTH LAUGH]. Um... [LONG PAUSE ... SIGHS] Shy, intelligent, [BRIEF PAUSE] queeeer [CHUCKLES], um... [I: W- was that one with a question mark?] No, no. [I: okay, okay .... BOTH LAUGH ... I: Just making sure]. Queer, um [LONG PAUSE] loving, [LONG PAUSE] Hm. Kind.

I: Great. So how hard was that? [LAUGHS]

P9: That was pretty hard [LAUGHS].

I: What was difficult about it?

P9: Um... [SIGHS] I don't know, 'cause there are so many [BRIEF PAUSE] different things that you can think about yourself and so many different ways that you can see it, [BRIEF PAUSE] that [SIGHS] trying to boil down all of your interactions and all of your different personality traits and quirks into five things is just kind of [BRIEF PAUSE] hard to do [CHUCKLES].

I: Yeah. Were there- are there words that you did not include that also describe your identity? 
P9: Um. [PAUSE] I guess, I think [BRIEF PAUSE] I become invested in things, uhh... [PAUSE ... SIGHS ... PAUSE] I am an anxious person [CHUCKLES]. [I: I am too ... BOTH CHUCKLE] Uhh... [PAUSE] I don't know...

I: So, did you deliberate at all in coming up with the first five?

P9: Sort of. Like there were some things where I would be like "oh, this could be kind of a substitute for this, or maybe I could say this one instead of that one", because you can say that they're synonyms, but they don't mean the same thing. [PAUSE] Uhh...

I: So how did you end up choosing certain words?

P9: [PAUSE] I just kind of tried to look at the way I interact with people, the way I picture myself. [BRIEF PAUSE] And the way I see things, and [BRIEF PAUSE] came up with those words [LAUGHS].

In the conversation that followed, the participant explained how one of the five identity words she listed, "shy", was a particularly important aspect of her identity, which led me to ask about the potential influence of minority stress experiences, in this case experiences related to stereotypes about sexual minority women, on her identity and identity development:

I: Right. So how have your, if at all, experiences around the stereotypes that we were talking about earlier, have they ever- have they been influential on that at all?

P9: I don't think so. I think that's [BRIEF PAUSE] me more than anything, because I kind of do my best to have the mentality that if somebody has [BRIEF PAUSE] a problem- a problem with my sexuality, then [CHUCKLES] they can 
leave, they are free to do that. [BOTH LAUGH ... I: Nice]. So I think that's more me than anything.

In my analysis of the relations among minority stress related codes (see ThirdPhase Inductive Thematic Analysis: Axial Coding) I found Participant-9's response here particularly interesting specifically because she expressed a lack of influence from minority stressors on her identity and experiences. Conversely, other participants who described their identities in terms of layers or hierarchies also described various minority stress related experiences, as highlighted in the previously quoted segments from Participant-5 and Participant-8.

Upon completing my analysis of the thematic patterns discussed here, I concluded that individuals who conceptualize their identities based on a hierarchy can be distinguished from those who are more concerned with defining the full extent of facets comprising their identities by the level of identity policing an individual has experienced from others. Identity policing is a code categorized under the minority stress sub-category of the intersectionality category and refers to experiences of having one's identity and expressions of identity policed or enforced by others around them. The set of participants' descriptions of their experiences coded as exemplifying or relating to this concept of identity policing clarified my earlier confusion regarding Participant-5's decision to exclude "sister" from her list of identity words due to the personal connotation she attributed to that part of experience (see Participant-5 excerpt above). Upon realizing the thematic pattern described here regarding minority stress and identity, I began to understand that an individual's identity could be experienced as something that is not 
inherently personal when an individual's agency in defining her own identity is compromised, as is a key component of the concept of identity policing.

In terms of the experiences shared by participants in this study, the impact of identity policing on an individual's own identity definition and/or experiences and behaviors depends in large part on the importance of the source of identity policing/enforcement. For some participants, having their identity and expressions of their identity policed in some way by family members was less impactful than experiencing identity policing from close friends or romantic partners, and for other participants it was the opposite (i.e., family members had the most impact). For example, Participant- 6 describes one of her experiences with identity policing, and the impact of those experiences, as follows:

P6: Um I did have- so when I first was like messing around with it, I had this friend at the time that was also queer, but was also like heavily into like the lesbian stereotypes and she would always be like "you can't go from like being fa femme one day to like being dyke-y the next day, like you have to- like you can only- you can just be one, like figure it out", and that was like [BRIEF PAUSE] really [BRIEF PAUSE] cr- I don't know that like affected me, I guess, so I sort of like slowly stopped like messing around with like my looks and just picked what like, not settled for or picked one, but started like- went back to like how I had always dressed.

The experience described by this participant not only illustrates the experience of having your identity or your expression(s) of your identity defined by others, but also the experience of identity policing from within the LGBTQ community. Interestingly, when 
Participant- 6 described experiences of identity policing from her own family members, she stated that she "took that more as a joke" (Participant-6), thus implying, in my analysis, that she experienced less of an impact from identity policing by her family members than she did by members of the LGBTQ community.

A second example of identity policing is illustrated in Participant-4's description of her struggle to define her own identity on her terms:

P4: Nobody expects people to evolve or change, unfortunately, other than like you know really basic things, uhm, but it's been more of the other I think where people expect you to identify this way and then that's who you are for the rest of your life, um... [BRIEF PAUSE] I definitely think- encountered that when I started identifying as queer instead of bisexual, um, [PAUSE] yeah... so... [BRIEF PAUSE] and then also with, you know, like race, I feel like that was also confusing being biracial [LAUGHS], I think that kind of- and then also the history of this country, I mean there's just a lot of things going on in my mind growing up where I was like, "Mexican? I'm not, I never lived in Mexico, like I was born here, but my par- my mom's from Mexico", and then like identifying as American for a while and I totally just not wanting anything to do with my Mexican heritage, and then finding an even ground between the two and yeah, that was just a huge evolution, well on the other hand my family still says I'm Mexican, they still want me to be Mexican, and yeah, like that for them isn't gonna change [BOTH LAUGH].

In sum, the common thread across participants who discussed experiences of identity policing from others is that the impact those experiences was (a) context- 
dependent (e.g., having such experiences while "coming out of the closet" was more impactful for many participants than identity policing experiences that occurred before coming out), and (b) typically more impactful when compounded either with other minority stressors (e.g., experiencing identity policing and discrimination at the same time) or with having multiple aspects of one's identity policed by others.

Collectively, these distinctions across participants and their experiences led me to broaden the definition of the minority stress construct within the intersectional model and this construct's positionality and interrelations with other constructs within intersectional model:

MINORITY STRESS as a construct within the modified intersectional model is defined as the loss or diminishing of choice regarding how an individual selfdefines and expresses their identity, or facets of their identity, due to having one or multiple marginalized identities. Minority stress is further conceptualized as a construct existing within the higher-level category of intersectionality.

Thus, within the framework presented here, experiences of minority stress are assumed to be multiplicative in that minority stressors are not understood as individual and mutually exclusive, but rather are intersectional in both the processes by which they are used against marginalized individuals and groups and in the extent of impact minority stressors have on an individual. This revised definition of minority stress for the working intersectional expands on how minority stress is typically operationalized in research and theory related to sexual and gender minority individuals' and groups' experiences (i.e., stress due to experiences of discrimination and prejudice based on one's sexual orientation; I.H. Meyer, 2003, 2010) by broadening the scope of possible minority stress 
experiences by conceptualizing the construct as part of the overarching category of intersectionality. Further this revised definition incorporates a mechanism potentially underlying the impact(s) of minority stressors on sexual minority women's experiences and identities by specifying the loss or diminishing of one's agency in defining and expressing one's identity. This latter specification is incorporated in the revised minority stress construct definition based on the thematic pattern regarding how individuals define and express their identities described throughout this section.

Gender role adherence and gender role stress construct definitions. Similar to the minority stress construct definition, gender-related experiences expressed by participants prompted an evaluation of the extent to which traditional definitions of gender role adherence and gender role stress sufficiently and accurately reflected sexual minority women's experiences and identities. It became apparent early in my data analytic process that gender roles and gender role adherence were defined and experienced among participants in this study in ways that were not reflected in the theories underlying these constructs. These underlying theories define gender roles as the social constructions of idealized gender-specific characteristics, attitudes, behaviors, and social practices to which groups and individuals are socialized to endorse, internalize, and aspire toward achieving. In addition, failure, or the potential of failure, to successfully adhere to these normative gendered prescriptions for individuals' identities and behaviors can result in experiences of gender role stress, and the level of stress experienced by an individual is theorized to vary in accordance with the individual's level of adherence to a particular set of gender roles (i.e., stronger adherence to a certain gender role coincides with higher levels of gender role stress; R. Connell \& Connell, 2005; Eisler et al., 2000; 
Moore \& Stuart, 2005). While past research has provided supportive evidence for these general definitions of the gender-related constructs of interest for this study, a salient theme among the data is the inner-connectivity between minority stress and gender role stress. Specifically, participants in this study evidenced experiences of gender role stress either as the result of minority stressors related to one or a combination of marginalized identities held by participants (e.g., marginalization based on gender, sexual orientation, race, or class identity), or as an additional form of minority stress they faced. In addition, based on the previously described open, focused, and axial coding analyses, the processes leading to experiences of gender role stress among participants tended to follow a particular pattern, which began with participants' fluidity in how they expressed their gender, whether intentional or not, led to experiences of gender role enforcement, which then led to experiences of gender role stress typically coupled with or indistinguishable from minority stressors.

Throughout her one-one-one interview, Participant-1's story exemplified the distinct process of gender role and minority stress experiences described above. For example, she was initially, and briefly, unable to name ways in which her gender identity and gender expression(s) influenced her day-to-day life; however, after only a couple of breaths this participant began detailing her experiences of "working in a man's world" (Participant-1). This participant then detailed how she uses her own gender identity and gender expressions in various ways, depending on the context, to either benefit her ability to obtain and maintain paid work or to cope with her work environments. For instance, she explained how she has "played-up" her femininity in order to get a job when being interviewed by a man, and then went on to a story of her experiences working on fishing 
boats and the need to perform just as well, if not better, than the men around her in order to keep her job. Further, this same participant began the interview (following the introductory activity) by discussing the influence of her family on her identity as a whole, her adherence to particular gender roles, her sexual orientation, and her experiences of intimate partner violence victimization from a former male-identified partner. For instance, Participant-1 mentioned her father telling her "... if you're going to act like a man you're going to be treated like a man" (Participant-1) in response to her actions in defending herself against her abusive husband then being arrested for that self-defense. Here, Participant-1 describes an experience in which her actions of self-defense are seen as deviations from what is expected of her according to her father's definitions of what "acting like a man" constitutes. She is then incarcerated as a consequence of her actions. Based on the combination of this participant's description of the context surrounding her use of violence in self-defense toward a previous intimate partner and her emphasis on her father's explicit gendering of her actions, I interpreted these experiences as being tied to her attempts to both achieve others' standards of her identity and behaviors while simultaneously holding steadfastly to her own agency. Further, through my inductive qualitative thematic analysis, I interpreted this participant's experience as a form of combined minority and gender role stress in that the participant evidenced minority stressors relevant to both her gender identity and her experiences of IPV victimization, as well as stressors relating to the externally imposed gender roles to which she was expected to adhere.

The processes evidenced by stories such as Participant-1's, combined with my previously described analysis and findings regarding participants' experiences related to 
minority stressors, led me to refine the working intersectional model's gender role adherence and gender role stress construct definitions in accord with data provided by this study's participants :

GENDER ROLE ADHERENCE is defined in the intersectional model as an individual's adherence to gender-specific expectations defined according to both the external influence of others' expectations of the individual, as well as the individual's internally-derived gender identity and expression(s).

Under this definition, gender role adherence refers not only to the act of adhering to a set of gender roles defined and enforced by others, as this construct is traditionally defined, but also to an individual's internally-based understanding and definition of her identity and how that identity is expressed.

GENDER ROLE STRESS is defined in the intersectional model as experiences of stress, strain, or internal conflict/confusion in response to situations in which one's internally-derived gender expression(s) deviates from what is externally expected of the individual.

This definition differs slightly from the original theoretically-based definition of gender role stress (i.e., stress in response to attempting or failing to live up to a particular set of gender roles) in that the experiences of stress in relation to gender, under the new definition, are not restricted to the individual's appraisal of a situation as stressful due to the weight of attempting to live up to a particular set of gender roles. Rather, the above definitions of both gender role stress and gender role adherence are broadened and distinguished from the traditional definitions to include the influence of gender-specific minority stressors. Similar to the revised definition of minority stress described above, 
these gender-specific constructs' revised definitions highlight the influence of externallyimposed expectations of how an individual identifies and expresses their gender. These externally-imposed expectations and their potential influences on sexual minority women's experiences and identities are represented in the Codebook as "Others Expectations", "Others Reactions", and "Others Confusion"; see Appendix C). The gender-specific definitions combined with the previously described definition of minority stress within the intersectional model were subsequently used to guide the application of the final codebook to the measures evaluated for RQ2.

\section{RQ2 Findings: Evaluation of Relevant Survey Measures}

While the first set of research questions addressed above are focused around the constructs and construct definitions within the working intersectional model, my second set of research questions (i.e., RQ2, RQ2a, and RQ2b) focused on evaluating a subset of the currently available survey measures that are designed to assess the constructs present within the intersectional model (see Table 1). RQ2, and its sub-questions, targets the evaluation of these measures on two points: (1) the extent of construct and content coverage provided by each measure in terms of sexual minority women's experiences and identities, and (2) the extent to which sexual minority women's experiences and identities are accurately represented by the construct definitions as presented by the content of each measure. In other words, in addressing the second set of research questions I sought to evaluate the sufficiency and accuracy of the content of measures currently available for assessing the intersectional model's constructs. To address RQ2, I applied the inductively derived codebook described above (see Appendix $C$ ) to ten of the twelve measures listed 
in Table 1 (see the previous section on RQ2 Analyses the rationale for excluding the CTS-2 and MGISF from further analysis).

A range of expectations was defined for how well the evaluated measures adequately and accurately cover sexual minority women's experiences and identities (see Figure 8). Specifically, I anticipated that the evaluated measures would fall along a spectrum of construct and content coverage and accuracy such that measures classified in the first panel of Figure 8 would lack any relevance to sexual minority women, while measures classified according to the second diagram of the fourth panel of Figure 8 would be encompassed by sexual minority women's experiences, but not fully representative of those experiences.

The majority of research to date using each of the evaluated measures often assumes that these measures are sufficiently representative of sexual minority women's experiences, and of those of the general population (O’Neil, 2008). This relationship between the experiences and measures is depicted in the third panel of Figure 8. However, based on my analysis of the available literature and the historical context of research related to intimate partner violence, gender roles, and minority stressors, I expected that the evaluated measures would be less representative of participants' experiences than the previous and current body of research related to these constructs has assumed. That is, I anticipated that most, if not all, of the evaluated measures would partially overlap with sexual minority women's experiences and identities, but that the measures would also either have irrelevant (or contaminating) content (i.e., Figure 8, Panel 2), or would be fully represented by SMW's experiences but not sufficiently 
representative of SMW's experiences, as shown in Figure 8, Panel Four (Keeley et al., 2013; Messick, 1995; Vogt et al., 2004).

Content coverage. In line with my above-described expectations, all of the relationships between the measures and women's experiences were best characterized by the fourth panel represented in Figure 8. Specifically, I found that the item sets of the ten evaluated measures (see Table 1) were fully encompassed by the content of the codebook (i.e., Figure 8, Panel Four); however, none of the measures fully covered all of the full set of codes within the codebook, and all but one of the measures were substantially deficient, overall, in terms of their content coverage of the codebook. The extent and nature of the overlap found among each evaluated measure and the inductively-derived codebook was determined based on the number of codes applied to each measure, as well as the frequencies of each code applied to each measure (see Table 8). Each of the measures evaluated in this study fell under one of four construct categories represented in the intersectional model: (1) gender expression (GREX), (2) gender role adherence (GRA), (3) gender role stress (GRS), and (4) minority stress. The content coverage of the codebook was relatively similar across the gender-related construct domains (i.e., GREX, GRA, and GRS) with the two gender expression measures (i.e., the Bem Sex Role Inventory and the Gender Expression Measure among Sexual Minority Women) collectively covering $24 \%$ of the codebook $(n=25$ codes $)$, the three gender role adherence (i.e., the Femininity Ideology Scale, Male Role Norms Inventory-Revised, and Social Dominance Scale) covering 28\% ( $n=29$ codes $)$, and the two gender role stress measures (i.e. the Feminine Gender Role Stress and Masculine Gender Role Stress scales) covering $20 \%(n=21$ codes $)$. The measures in the minority stress category (Mohr 
\& Fassinger, 2000; Pinel, 1999; i.e., the Lesbian Internalized Homophobia Scale, Outness Inventory, and Stigma Consciousness Questionnaire; Szymanski \& Chung, 2008) showed considerably greater combined coverage of the codebook (i.e., $43 \% ; n=44$ codes); however, this coverage is primarily attributable to the Lesbian Internalized Homophobia Scale (Szymanski \& Chung, 2008), which covered 31\% of the codebook ( $n=32$ codes) on its own, while the Outness Inventory (Mohr \& Fassinger, 2000) covered only 17\% of the codebook and the Stigma Consciousness Questionnaire (Pinel, 1999) covered only $14 \%$ of the codebook. Visual representations for the combined overlap across measures originally designed to assess these four construct domains within the working intersectional model are provided in Figure 9, and Figure 10 depicts the combined coverage of the codebook across all measures evaluated in this study.

Content deficiencies. The list of codes, organized by their corresponding construct categories, not covered by any of the evaluated measures is provided in Table 9. Similar to the actual content coverage observed among the measure for the codebook, there appears to be a relatively equitable distribution of content deficiency across the codebook's Minority Stress and Gender categories across the evaluated measures. Of particular import to the goals set of study is the relative consistency with which the collective set of measures lacks coverage of intersectional experiences and identities. For example, the inductively-derived codes "Priority Identity", "Privilege", "Race", "Race Identity", and "Intersectionality- $X^{\prime \prime}$ (i.e., intersectional marginalization) were not covered in my application of the codebook to the measures. Yet, these were sometimes the most salient themes in this study's participants' expressed experiences and identities. Thus. while I found evidence suggesting that the content of the evaluated measures is relevant 
to sexual minority women's experiences and identities as expressed by this study's participants, there remains a deficit in both the overall breadth of coverage provided by the set of measures evaluated here, as well as the accuracy with which SMW's experiences and identities are represented by the measures.

Inter-rater reliability. As previously described, a second trained coder also applied the codebook to each measure, and an analysis of the agreement using Cohen's Kappa between mine and the second coder indicated an overall moderate level of agreement (see Tables 6 and 7). While I accepted this level of agreement for the purposes of the current study, I also interpreted this as an indication that the codebook could potentially use additional refinement in both the number of codes and the definitions of the codes. This conclusion is based on the common interpretation of low-to-moderate inter-rater reliability indicators that a codebook's composition (i.e., code definitions and scope of the codebook) and/or implementation (i.e., application to observations) is not consistently agreed upon (McHugh, 2012).

The findings presented above reflect the measures' coverage of the codebook according to the codes applied by both myself and the trained research assistant. In my first independent iteration of applying the codebook to the measures, I found that seven of the ten evaluated measures partially covered the codebook's content, but were neither fully encompassed by nor encompassing of the codebook, thus reflecting partial content coverage of the experiences and identities of sexual minority women represented by this study's participants (i.e., Figure 8, Panel Two). The content within each of the ten measures not encompassed by the codebook reflects potential content contamination (i.e., content present within the measure that is not relevant to the respondents and can 
potentially hinder a given measure's reliability and/or validity). However, the small sample size and lower than ideal inter-rater reliabilities prevent any concrete conclusions in this regard. 


\section{Chapter 7. Discussion}

The present research serves as the foundational study for a larger program of research aimed at informing intimate partner violence (IPV) prevention and intervention strategies specific to sexual minority women (SMW). The overarching purposes of this study were to (1) inform the definitions and implied relations among the constructs currently present in a working intersectional model predicting same-gender IPV perpetration among women (see Figure 2), and (2) evaluate the content validity and construct domain coverage of a subset of pre-existing gender, minority stress, and IPVrelevant survey measures to determine their efficacy at assessing sexual minority women's experiences and identities (see Table 1 and Figure 8). In line with the exploratory nature of these purposes and the research questions listed in Chapter 4, this study was conceptualized under a qualitative emergent design. While the emergent design was primarily implemented to emphasize the discovery-oriented goals of the larger program of research within which the present study is situated, this study was also designed to directly inform the development of a theoretical model predicting IPV perpetration among sexual minority women (see Figure 2), which will be tested in subsequent phases of the larger program of research (Morgan, 2014, Chapter 6). This necessitated an evaluation of the constructs present within the model in terms of their operational definitions and the implied relations among the constructs. This further necessitated an evaluation of the level of accurate and relevant representation of sexual minority women's experiences and identities present in the currently available survey measures for assessing the model's constructs among sexual minority women. Thus, this study was also designed to address the development and definition-oriented purposes 
collectively reflected in the study's research questions and overarching goals (see Research Questions in Chapter 5; Morgan, 2014, Chapter 6). The analytic procedures employed to address each of these research questions and corresponding key findings are briefly reviewed below, followed by details regarding the implications and limitations of those findings as they relate to IPV-specific theory, research, and intervention and prevention strategies.

\section{Intersectional Model Construct Definitions and Relations Evaluation}

The underlying goal of the first research question (i.e., RQ1: To what extent are sexual minority women's experiences and identities accurately captured by the constructs present in the intersectional model adapted from R. M. Smith et al. (2015)?) was to yield clarity in the experiences and identities of sexual minority women with specific attention given to experiences related to the constructs present within the working intersectional model depicted in Figure 2. To this end, the qualitative interview and focus group data collected for this study were used to develop a codebook to inform the working intersectional model's construct definitions and implied relations in order to assess the accuracy and quality of the working intersectional model in terms of its utility in informing IPV intervention and prevention strategies specific to LGBTQ women.

I developed the 103-item codebook provided in Appendix $C$ through a set of systematic inductive qualitative analyses based in Grounded Theory (GT) methods (Charmaz, 2011, 2014; Corbin \& Strauss, 1990; see Chapter 5; Strauss \& Corbin, 1994). The codebook's collective content (including the extent of the codelist, the codenames themselves, and the code definitions) is the result of combining theoretically-driven concepts with this study's qualitative interview and focus group data to form a cohesive 
and comprehensive representation of participants' experiences and identities. Further, the codebook's organization into four overarching categories (i.e., Gender, Identity, Minority Stress, and Intersectionality), is grounded in the participants' organization of each concept, rather than the semantic meaning of the concept represented in each codename. This is particularly evident in the Intersectionality category's three sub-categories, all of which reflect intersections among the Gender, Identity, and Minority Stress codebook categories. Once the codebook's content reached a point of saturation in terms of its coverage of the qualitative data collected for this study, I evaluated the intersectional model's constructs in terms of their original conceptual and operational definitions and the existing theories and research evidence underlying those original conceptualizations. This construct evaluation yielded modified definitions for the model's overarching construct domains:

MINORITY STRESS: The loss or diminishing of choice regarding how an individual self-defines and expresses their identity, or facets of their identity, due to having one or multiple marginalized identities. Minority stress is further conceptualized as a construct existing within the higher-level category of intersectionality. GENDER ROLE ADHERENCE: Adherence to gender-specific expectations defined according to both the external influence of others' expectations of the individual, as well as the individual's internally-derived gender identity and expression(s). GENDER ROLE STRESS: Experiences of stress, strain, or internal conflict/confusion in response to situations in which one's internally-derived gender expression(s) deviates from what is externally expected of the individual. 
Minority stress theory implications. I. H. Meyer (2003) developed a foundational framework for defining and investigating sexual minority stress, its antecedents, and its consequences. Under this framework, minority stress is generally defined as: "... related to the juxtaposition of minority and dominant values and the resultant conflict with the social environment experienced by minority group members" (I. H. Meyer, 1995, p. 39). Research conducted using this overarching theoretical framework has operationalized minority stressors as experiences of prejudice, discrimination, violence, and/or social stigma perpetrated against individuals based on their minoritized social positions (I. H. Meyer, 2003, 2010). The intersectional model's amended minority stress construct definition compliments and extends, rather than contradicts, this prevailing minority stress theoretical framework specific to sexual and gender minority populations (I. H. Meyer, 2003, 2010; Szymanski, Chung, \& Balsam, 2001). Figure 11 provides I.H. Meyer (2003)'s original theoretical model, while Figure 12 depicts an expansion of Meyer's model based on this study's minority stress findings and their implications. The modified minority stress definition focuses on the loss or diminishment of choice regarding an individual's self-definition(s) and expression(s) of her identity as an additional mechanism potentially underlying or consequenting from experiences of minority stress among sexual minority women. Within the context of I. H. Meyer's conceptual model, the amended minority stress definition lends a route toward behavioral consequences of minority-stress-related experiences, as depicted in Figure 12, in addition to the mental health outcomes posited as the final point in I. H. Meyer (2003)'s original conceptual model (see Figure 11). 


\section{Influence of minority stress on gender role adherence and gender role stress.}

The qualitative findings related to participants' experiences of minority stress, and the impacts of those experiences on participants' identities and behaviors, were surprisingly influential in my analysis of participants' gender role adherence and gender role stress experiences. That is, participants' responses to gender-role-specific interview and focus group questions and prompts consistently suggested that their experiences and identification with gender roles, of any gendered type (i.e., androgynous, feminine, masculine, non-binary gender roles, etc.), were in many ways tied to experiences of minority stressors. This was particularly salient within the context of the revised minority stress definition developed as a result of the inductive qualitative analyses. The construct definitions for gender role adherence and gender role stress were thus revised for the working intersectional model to emphasize the interplay among externally-imposed expectations of how an individual internally identifies and expresses their gender. The resulting gender role adherence and gender role stress construct definitions within the intersectional model focus on the impact of minority stressors, in the form of a loss or diminishing of choice regarding one's identity and/or expressions of identity.

In my previous review of the literature related to the intersectional model's constructs (see Chapter 3), I argued that members of systematically marginalized groups may internalize and adhere to the same cultural norms that function as tools for oppression. I further posited that sexual minority women who identify with masculinity may internalized the normative beliefs associated with traditional hegemonic masculinity, which may elicit violent and/or manipulative behaviors toward their female intimate partners in response to experiences of gender role stress, and that such responses may 
also be impacted by experiences of minority stress. This study's findings provide qualitative support for the latter component of this possible process, as well as evidence that sexual minority women's minority stress experiences may be much more interwoven with their experiences with gender roles and corresponding behavioral responses than my original conceptualization represents.

Minority stress and gender roles in female same-sex IPV research. The revised intersectional model's gender-related construct definitions extend the scope of hegemonic masculinity theory's applications in IPV research (R. Connell, 2002; R. Connell \& Messerschmidt, 2005; Mankowski \& Maton, 2010). The role of minority stress experiences in the operationalization of the gender role adherence and gender role stress constructs is situated in the meanings of choice and control among sexual minority women and their experiences with these gender-specific constructs. That is, hegemonic masculinity has predominantly been considered within the context of hegemonically masculine men, with hegemonic masculine gender role stress typically examined with regard to such men's ability, or lack thereof, to achieve and/or maintain their hegemonic masculine status (R. Connell, 2002; R. Connell \& Connell, 2005; R. Connell \& Messerschmidt, 2005; Mankowski \& Maton, 2010; Moore \& Stuart, 2004, 2005; Moore et al., 2010). Conversely, the revised definitions of the intersectional model's genderspecific constructs suggest that sexual minority women's gender role stress experiences may manifest as stress resulting from losing, or never experiencing, a sense of control over one's own gender role identity and gender expression due to their marginalized identities. Thus, the revised gender-specific construct definitions suggest the need to further investigate the unique qualities of sexual minority women's gender role adherence 
and gender role stress experiences. The interconnectivity evidenced in participants' gender-role-related and minority stress experiences warrants a deeper integration of these construct categories in future IPV-specific research and intervention efforts specific to sexual minority women.

IPV-specific theory, research, and interventions are most often based in a theoretical and research tradition identifying power, dominance, and control as the underlying motivations for IPV perpetration (Johnson, 2006; Pence, 1983; Sokoloff \& Pratt, 2005). Within this understanding of IPV are the previously reviewed (see Chapter 3) theories related to hegemonic masculinity and the relationship between endorsement of and adherence to hegemonic masculine norms and heterosexual men's IPV perpetration (Moore \& Stuart, 2005; Murnen et al., 2002; R. M. Smith et al., 2015). Combining these existing understandings of IPV perpetration with the modified minority stress construct definition developed through this study's inductive thematic analysis provides an additional framework for understanding IPV perpetration, particularly in the context of same-gender IPV among sexual minority women.

The modified gender role adherence and gender role stress construct definitions are each focused around the tensions between gender-related, externally-imposed expectations (i.e., through social constructions and enforcements of arbitrary, yet rigidly defined, gender-specific social categories) and an individual's self-defined and/or selfchosen gender identity, gender role, and/or gender expression. Given the modified minority stress construct definition's explicit focus on the impact of losing control or agency over one's own identity definition and expressions, the modified gender-related construct definitions can be further understood as conceptually adjacent to minority 
stressors in terms of their focus on an individual's agency in determining her own genderrelated identities, roles, and/or expressions. As such, sexual minority women's experiences of minority stressors may manifest in ways that critically impact their gender role adherence and gender role stress experiences, which may in turn influence their likeliness or unlikeliness to perpetrate IPV toward their same-gender partners. Specifically, there is a thematic cohesion centered around the issue of control across participants' expressed minority stress and gender-related experiences, as is reflected in the conceptual cohesion across the modified construct definitions. The qualitative findings regarding the interplay among minority stress, gender role adherence, and gender role stress indicate a need for future IPV-related research to develop and incorporate psychological measurement strategies and tools assessing sexual minority women's perceptions of, motivations, and tactics for establishing and maintaining control over their own identities and expressions. Future research is further needed that examines whether these motivations and tactics for maintaining control might also influence their perpetration, or non-perpetration, of same-gender IPV.

\section{Measurement Evaluation}

The above-discussed RQ1 findings guided and facilitated the measurement evaluation analyses conducted to address RQ2 (i.e., To what extent are sexual minority women's experiences and identities adequately captured in survey measures developed to assess the constructs in the intersectional model?). The inductively-derived codebook was applied to ten of the thirteen preexisting survey measures listed in Table 1 (see $R Q 2$ Analysis in Chapter 5 for further explanation regarding the exclusion of the Masculine Gender Identity Scale for Females and Revised Conflict Tactics Perpetration and 
Victimization Scales). Each of the evaluated measures was designed as to assess the constructs present in the working intersectional model (see Figure 2) and was evaluated according to the extent of each measure's content coverage of the codebook. The measures include in this evaluation were selected based on (a) the relevance of each measure's target construct(s) to the intersectional model's construct domains (i.e., gender role adherence, gender role stress, and minority stress), and (b) the extent and quality of pre-existing evidence of each measure's construct and content validity and reliability. To this end, I reviewed the available literature related to each of the constructs present in the intersectional model to find any measures relevant to each construct, and then reviewed the available construct and content validity and reliability evidence for any such relevant measures. This review began with the original publications for each measure's development as well as any subsequently published investigations of each measure's validity and/or reliability. The resulting list of measures selected for evaluation provided in Table 1 showed adequate relevance to the intersectional model's constructs, as well as promising evidence of validity and reliability.

Findings from this analysis suggest that, while the overall set of measures does not fully encompass the codebook, all of the evaluated measures contain some degree of relevance to sexual minority women's experiences and identities (see Table 8 and Figures 9 and 10). This conclusion is based on the finding that each of the ten measures was fully encompassed by the codebook's items such that both myself and the research assistant serving as the second coder for the measures evaluation analysis agreed that no measure contained items that could not be conceptually represented by at least one of the inductively-derived codebook's items. There was, however, a distinction regarding the 
level of codebook coverage found among the four measurement categories (i.e., Gender Expression, Gender Role Adherence, and Gender Role Stress, and Minority Stress) such that the combined set of minority stress-related measures showed more coverage than that found among each of the gender-related measurement categories (see Table 8 and Figure 9). This discrepancy in the construct domain coverage across measures is not especially surprising, given that all of the minority stress measures were specifically designed for use with sexual minority women, whereas only one of the gender-relevant measures (i.e., the Gender Expression Measure among Sexual Minority Women; Lehavot et al., 2011) was designed for this population. Collectively, these findings regarding the evaluated measures inform both the current level of accuracy with which SMW's experiences and identities are represented by the evaluated measures, as well as the adaptations that could be implemented to improve assessments of sexual minority women's experiences with the intersectional model construct domains. These measurement-specific implications are detailed below.

\section{Suggested modifications to the evaluated measures and directions for future}

measurement-specific research. While the above measurement evaluation findings suggest that the evaluated measures may collectively provide a minimally sufficient level of content coverage in terms of assessing sexual minority women's experiences and identities, there remain key components of the codebook not covered by the evaluated measures (see Table 9). For instance, one of the codes not covered by the measures in this study's analysis was "Priority Identity" (see code definition in Appendix C), which reflects the pattern of defining one's identity according to a hierarchy in response to experiences of minority stressors (see box labeled " $(g 1)$ Characteristics of Identity 
Definition" in Figure 12, and RQ1 Findings in Chapter 6 for further details on the "Priority Identity" concept). The "Intersectionality" code was also not covered by the evaluated measures' content, yet the saliency and meaning of the "Intersectionality" code throughout my inductive analysis of the interviews and focus group content led to its inclusion as an overarching category in the codebook (see Appendix C). Further, the "Intersectionality- $X$ ", or the concept of "intersectional marginalization", which reflects a form of minority stressors wherein oppressions are experienced and perpetrated from within an already marginalized population or community (e.g., transphobia among lesbian women and gay men, issues of racism within the LGBTQ community, etc.) was also not covered by any of the evaluated measures. The interview and focus group data coded with the "Intersectionality- $X$ " code reflects myriad experiences in which oppressions perpetrated toward participants by other members of the LGBTQ community heavily influenced participants' later beliefs, attitudes, and behaviors.

The lack of content within the evaluated measures reflecting experiences of intersectional marginalization is particularly important within the context of IPV-related research specific to, or inclusive of, sexual minority women. The perpetration of samegender IPV among women can also be understood as a form of intersectional marginalization. Incorporating items that inform the level complexity of respondents' experiences with minority stressors beyond, but potentially intersecting with, stressors specific to sexual orientation and gender could improve the content coverage for the minority stress measures. For example, respondents' agreement with the Lesbian Internalized Homophobia Scale's third item, "I feel isolated and separate from other lesbians" (Szymanski \& Chung, 2008), could be a reflection of either or both internalized 
homophobia, in the form of feeling disconnected from one's identity-specific community, or experiences of discrimination, in the form of isolation, within the lesbian community (i.e., intersectional marginalization, or "Intersectionality- $X$ " in the codebook; see Appendix $C$ ). Asking more about the context in which respondents do or do not experience isolation and other forms of oppression among other sexual minority women could improve the accuracy and extent of information provided by this item (e.g., "I feel like I am the only person of my race in my lesbian/LGBTQ community", and/or "Lesbians look down on my bisexuality"). The latter suggested items are worded according to evidence from this study's participants wherein (a) racial and ethnic minority participants shared experiences of discrimination and isolation based on their race and/or ethnicity from within their LGBTQ communities, and vice-versa (e.g., racism experienced among other LGBTQ groups and individuals, homophobia/heterosexism experienced from other racial and ethnic minority groups and individuals, etc.), and (b) bisexual participants emphasized experiences of discrimination and isolation by lesbian-identified women in their lives based on their sexual orientation (see RQ1 Findings in Chapter 6). Additional research is needed to further develop the definition of intersectional marginalization as a psychological construct in order to facilitate the development of accurate and reliable measurement approaches for assessing this concept among historically minoritized populations, including, but certainly not limited to, sexual minority women. Based on the present study's exploratory findings, research efforts in this direction will be especially useful in terms of further expanding the current understandings of and theoretical frameworks regarding the IPV-related antecedents, correlates, and consequences of minority stress experiences among marginalized populations. 
Points of view reflected in the measures' items. There is also an interestingly subtle difference between measures designed specifically for use with sexual minority women populations and those designed with the general population in mind: the point of view represented in the measures' items. That is, all of the measures specifically designed to assess sexual minority women's experiences and/or identities use a first person perspective, for example:

"I wish some lesbians wouldn't "flaunt" their lesbianism. They only do it for shock value and it doesn't accomplish anything positive" (Lesbian Internalized Homophobia Scale, Item-47; Szymanski \& Chung, 2008).

"I am usually physically protective of my partner or date" (Gender Expression Measure Among Sexual Minority Women, Item-2; Lehavot et al., 2011). Conversely, other measures use mostly third person perspectives:

"A girl should be taught how to catch a husband."; "Women should dress conservatively so they do not appear loose." (Femininity Ideology Scale, Items 31 \& 35, respectively; Levant et al., 2007).

"Men should get up to investigate if there is a strange noise in the house at night" (Male Role Norms Inventory-Revised, Item-27; Levant et al., 2010). As previously mentioned, the greatest amount of content coverage of the codebook was observed among measures designed specifically for sexual minority women (see Table 8 and Figure 9). The difference in perspective reflected in each of the evaluated measures' items could also contribute to the measures' assessed relevance and accuracy to the codebook's content. Given that the inductively-derived modified definitions of the major construct domains present within the intersectional model (i.e., gender role adherence, 
gender role stress, and minority stress) each emphasize the tensions between internal versus external influences on individuals' identity definitions and expressions, the point of view reflected in a given measure's items may also contribute to the measure's relatability to sexual minority women's experiences and identities. Thus, greater coverage of the experiences and identities represented in the codebook developed in this study might be further achieved by modifications to the point of view of the measures' items. For instance, the Male Role Norms Inventory-Revised (MRNI-R) example item provided above could be revised as "I feel the need to get up to investigate if there is a strange noise in the house at night", or "It is my responsibility to get up to investigate if there is a strange noise in the house at night". Within the context of a measure designed to assess endorsement of cultural norms, participants' responses to items like these could collectively reflect endorsement of, and adherence to, hegemonic masculine norms according to both this study's modified gender role adherence construct definition, as well as Levant et al. (2010)'s operationalization of the construct. Further, an especially relevant concern regarding the MRNI-R's, and others', use of a 3rd-person perspective for the majority of its items is that it causes the measure to focus exclusively on men's roles rather than on a hegemonic masculine role because the items are worded as "Men / A man should ...". A core component of the present research, however, is focus on women's internalization of and adherence to normative hegemonic masculine roles. With either of the alternative items provided here, the original MRNI-R item (Levant et al., 2010) retains both its contextual meaning and its capacity for capturing the target construct (i.e., adherence to or endorsement of the "Toughness" domain of hegemonic masculinity), while also extending the item's binary scope to include individuals who do not identify as 
men. Such an extension might allow, for instance, sexual minority women to more closely or realistically relate to the behavior and opinion reflected in the item (Levant, Hall, \& Rankin, 2013; Levant et al., 2010).

\section{Limitations}

The extent of the implications and contributions of this study's findings is bound by the limits of the study's design and implementation. This study exists as the foundational qualitative input design for a multiphase mixed-methods program of research investigating the mechanisms and root causes underlying sexual minority women's perpetration of abuse toward their same-gender partner. Given the exploratory nature of the study's design, no hypotheses were tested regarding the constructs of interest or the survey measures evaluated in this study. As such, the findings can be interpreted within the bounds of the discovery-, development-, and definition-oriented utilities of the study's qualitative emergent design, and its theory-building and hypothesis-generating purposes (Morgan, 2014).

Sample size and characteristics. The study's sample size and sample characteristics are limited to the number of eligible participants that were successfully recruited for participation using both purposive and convenience sampling methods. In the planning stages of this study, the expectations for addressing the first research question focused heavily on the methodology and, more specifically, the interview structure and participant composition originally planned for this study. However, methodological changes were later implemented due to difficulties in recruiting enough individuals meeting the specified criteria for each focus group who would be available to attend a focus group on the same day and time. As such, this study's methodology shifted 
away from strictly recruiting focus group participants, and toward recruiting both one-onone interview participants as well as potential focus group participants. This methodological shift ultimately yielded ten one-on-one interviews and one focus group comprised of four participants, amounting to the final sample of fourteen participants.

The sample size is somewhat smaller than originally anticipated for this first series of focus groups and one-on-one interviews. This limits the extent of conclusions that can be made regarding the level of coverage provided by both the constructs present within the intersectional model and the evaluated measures. Further, the nature of conclusions possible regarding the content of the inductively-derived codebook developed to evaluate the intersectional model and pre-existing survey measures is bound by the study's sample size and participant characteristics.

While the original study design included conducting focus groups with sexual minority women currently receiving services related to intimate partner violence victimization and perpetration, the purposive sampling efforts for these participant categories were not successful. The final sample does include individuals with histories of female same-sex intimate partner violence victimization and perpetration; however, the fact that these participants were not directly recruited through an organization from which they were currently receiving IPV-related services impeded my ability to assess participants' experiences with IPV victimization and/or perpetration. Further, the IPVspecific purposive sampling efforts were least successful in recruiting individuals for the IPV perpetration category such that three $(21 \%)$ of the 14 participants reported having perpetrated IPV toward a former intimate partner, and two of those three reported having perpetrated against a female-identified former intimate partner. This limits the ability to 
make conclusions regarding potential differences in experiences and behaviors among participants who have and have not perpetrated IPV. Importantly, the low sample of IPV perpetrators is in large part due to the lack of IPV perpetration intervention services available to women who perpetrate IPV. That is, while there is one IPV victimization program available in the Portland, OR area that specifically focuses on LGBTQ individuals (i.e., Bradley Angle's LGBTQ IPV victimization response program in Portland, OR), there is no such program locally available for LGBTQ women who perpetrate IPV.

The inductively-derived codebook's content is strongly grounded in the experiences and identities shared by the fourteen sexual minority women recruited from the general Portland, Oregon area. Just over half (eight out of fourteen) of the sample identified their race or ethnicity as White or Caucasian, while $43 \%$ of participants indicated their race and/or ethnicity as multiracial identities; the average age represented in this sample is 32 years $(S D=12$ years); and the majority of participants were in a romantic relationship at the time of their participation (71\%; see Table 4). Further, all of this study's participants also indicated that they moved to Portland, OR from another state, and that the places they lived prior to moving to Portland were at least as urbanized as Portland, OR, if not more-so. This is not especially surprising given the continuous population growth within and around the general Portland, OR area; however, this also limits the study's sample to not include individuals who either (a) grew up in the Portland, OR area for the majority of their lives, or (b) lived in more rural locations. Using the same Grounded Theory-based analytic procedures employed in this study (see RQ1 Analyses in Chapter 5) with a larger and more diverse sample of sexual 
minority women, the extent of the actual codelist could either grow or shrink and the code definitions might differ from their current states in this study's codebook (see Appendix ). For instance, given a larger sub-sample of sexual minority women with histories of same-gender IPV perpetration, the codebook's content could become more focused in ways that better differentiate between sexual minority women with histories of same-gender IPV perpetration and those with no such IPV history. This differentiation might emerge through distinguishing qualitative patterns in sexual minority women's minority stress and gender-related experiences, such as the emergent thematic pattern of hierarchical versus faceted identity definitions found among this study's participants. Further, incorporating perspectives of additional participants with histories of samegender IPV victimization and/or perpetration might provide insights regarding the "IPV" code's definition, which currently is just "Intimate Partner Violence" due to the lack of detailed content regarding the nature and dynamics of participants' expressed IPV-related experiences. That is, while several one-on-one interview participants mentioned IPV experiences during the interviews, they did not offer more specific details on the nature and dynamics of those experiences, and IPV was not present as a thematic topic in the focus group's content. Additional focus groups and one-on-one interviews might therefore yield information to fill the gaps in this study's IPV-related qualitative data. Modifications to the intersectional model, beyond or potentially including the construct definitions themselves, may become necessary upon conducting an additional set of interviews and analytic procedures similar to those done for this study. Further, the content coverage of the codebook found among the measures may shift given additional perspectives from a large and more diverse sample of sexual minority women. These 
possibilities regarding the codebook's content impact the extent and nature of conclusions regarding the codebook's utility as a tool for evaluating both the working intersectional model's and the corresponding pre-existing survey measures' construct relevance and content coverage among sexual minority women.

Measurement evaluation analysis limits. While the codebook's overall observed coverage of the evaluated measures' content suggests that few adaptations are needed in terms of the existing items in each measure, this coverage was only evaluated and observed in terms of the conceptual content of each measure's items. That is, in applying the codebook to the measures, the research assistant serving as the second coder and I only applied a code to a given item if the code could conceptually replace that item's content. While this limits our ability to definitively decide whether specific items should be modified or removed entirely from a given measure, the conceptual application of the codebook to the measures in this study was intentional given the exploratory nature of this study's goals and the design of the larger program of research for which this study represents the first phase of inquiry. That is, the immediate next step for this particular project will be to conduct a second set of interviews and focus groups using a more structured interviewing strategy in which participants' evaluations of both the conceptual content and presentation (e.g., verbiage, readability, relatability, etc.) of a given measures' set of items will further inform the measurement evaluation initiated in this study.

\section{Reflections on Current IPV Intervention and Prevention Practice}

It may be that it simply takes the passage of a mandatory-arrest law to convince officers that women can be the victims of other women in domestic situations. (Pattavina, Hirschel, Buzawa, Faggiani, \& Bentley, 2007, p. 388) 
There remains a need to understand the extent and nature of IPV among sexual minority women within the contexts of their local communities. Currently, the available body of research specific to or inclusive of sexual minority women's experiences of same-gender IPV in the United States remains primarily focused on nation-level or stateto-state comparison estimates of IPV among sexual minority women. However, inclusive and effective IPV intervention services, both in terms of perpetration and victimization, for sexual minority women currently in need of such services remain largely unattainable.

A core goal of this study, as well as the larger program of research in which it is situated, is addressing sexual minority women's perpetration of same-gender IPV from the local community's perspective(s). Over the past three years, this community-engaged approach includes my active participation in various Oregon-based organizations and networks comprised of community-based IPV and sexual violence intervention and prevention practitioners (e.g., serving as a member of the Oregon Attorney General's Sexual Assault Task Force's Prevention and Education Committee, regularly attending the Tri-County Network of Batterer Intervention Providers' Network monthly meetings). The present study's goals, research questions, design, and interpretations of findings are strongly influenced by questions asked and information provided by the practitioners I regularly interact with through these community-based activities. My reflections on these observations in the context of this study's findings and corresponding implications are discussed below.

When asked why they do not provide services specific to, or inclusive of, sexual minority women multiple batterer intervention providers in Oregon have stated that their organizations have yet to experience the demand for such specificity in their services to 
warrant providing them. However, recent estimates from the National Intimate Partner and Sexual Violence Survey (NISVS) indicate that $67 \%$ of lesbian women who experienced rape, stalking, and/or physical abuse by an intimate partner at least once in their lifetime also reported only female perpetrators (M. C. Black et al., 2011; Walters et al., 2013). These NISVS findings provide evidence that same-gender IPV among sexual minority women occurs at rates comparable to those found among women in heterosexual relationships (see Chapter 1). Combining these nation-level prevalence findings with state-level IPV victimization estimates from the same national survey (M. C. Black et al., 2014), these percentages translate to an estimated 21,174 women who perpetrate abuse toward a female intimate partner in Oregon each year. While the scope of the present study's purpose does not specifically include analysis of the prevalence of IPV experiences among sexual minority women, this study's participant recruitment processes generated additional evidence supporting these prevalence estimates. In particular, the initial participant recruitment efforts for this study's IPV-specific participant categories (see Table 2) proved very difficult when these efforts were focused strictly on recruiting participants directly from community-based IPV service providers. However, the nonIPV-specific sampling efforts (see Figure 6) in fact yielded the entirety of this study's IPV-specific participant categories' sub-samples. This indicates that FFSIPV is occurring but may not commonly be identified in samples recruited through community-based IPV services. Further, a notable characteristic of the IPV-perpetration participant category of this study's sample is that all participants who reported past perpetration of same-gender IPV also reported past experiences of IPV victimization (see Tables 3 and 4). 
The disconnect between community-based service providers' experiences and the available evidence of the prevalence of IPV among sexual minority women is in part due to the continued distrust many LGBT individuals have of the criminal justice and mental health systems (Mallory, Hasenbush, \& Sears, 2015; Mogul, Ritchie, \& Whitlock, 2011). Further, there remains a lack of recognition of incidents of FSSIPV within the criminal justice and mental health systems, and the knowledge of this can be both a barrier to a current victim seeking help through these systems, as well as a tool for a perpetrator to use against the victim to prevent reporting. That is, telling the partner that no one will believe her if she reports the abuse has been evidenced in past research within the developing body of research regarding women's same-gender IPV perpetration tactics (Hassouneh \& Glass, 2008; Pattavina et al., 2007). The above-described contexts, issues, and research evidence collectively necessitate a comprehensive awareness and recognition of control and manipulation tactics that might be used by IPV perpetrators, regardless of gender. The existence of female same-sex IPV is not new, rather its existence is becoming increasingly recognized as (a) real and (b) in need of attention. The previously described prevalence of FSSIPV indicates that the need for intervention services does in fact exist in Oregon, but that the individuals in need of these services are not being recognized by the system.

Another issue specifically and consistently named by community-based service providers across Oregon is that their organization is not equipped with the information or evidence base for providing appropriate and effective services to sexual minority women populations. As a community-engaged researcher with an action-oriented outlook toward preventing IPV, this concern among Oregon service-providers served as an indication 
that nation-level estimates and state-to-state comparisons, while unquestionably valuable in the context of public policy and funding allocations, are not sufficient for informing the IPV intervention and prevention work on the ground. Further, the consistency with which the lack of practical research information negatively impacts IPV-specific service providers' ability and confidence in providing services specifically to sexual minority women reiterates the need for more community-based research efforts, both in Oregon and elsewhere, that precisely incorporate practitioners' questions and needs in their designs and implementations.

\section{Conclusions}

Findings from this study contribute to an understanding of the extent to which sexual minority women's experiences are sufficiently and accurately represented in both a working intersectional model predicting SMW's same-gender IPV perpetration (see

Figure 2) and a set of evaluated pre-existing and widely used measures designed to assess the model's constructs (see Tables 1). The study was designed to develop an empirically practical foundation for a larger program of research investigating the possible relations among hegemonic masculine gender role adherence, gender role stress, minority stress, and same-gender intimate partner violence (IPV) perpetration among sexual minority women. The overarching goal of this program of research is to inform IPV intervention and prevention strategies specific to sexual minority women. Due to the relative lack of empirical knowledge around the prevalence and causes of intimate partner violence among Lesbian, Gay, Bisexual, Trans, and Queer (LGBTQ) populations, this goal could not be realistically met without first grounding this program of research in the 
experiences and identities of the population of interest (i.e., LGBTQ/sexual minority women).

While theoretical and empirically evidenced explanations exist regarding IPV among heterosexual couples, primarily in terms of men's IPV perpetration toward women, there is relatively little empirical research available regarding the limited array of theoretical explanations for women's perpetration of violence in their same-gender relationships. Because the IPV-related body of research, including more general psychological research related to personality and interpersonal relationships, is heavily focused on the experiences and identities of heterosexual individuals, the tools available for empirically assessing the various components of a given theoretical explanation for sexual minority women's perpetration of same-gender IPV are limited in their utility with this population. These concerns regarding the scope and content of the available IPVrelated research literature motivated the designs of both the current study and the overarching multi-phase program of research in which this study is situated. In particular, the available research related to IPV among sexual minority women has thus far been limited in quantity and consistency in methodological rigor, as evidenced by BadenesRibera, Frias-Navarro, Bonilla-Campos, Pons-Salvador, \& Monterde-i-Bort (2015)'s struggle to obtain a substantial sample of studies to conduct the first known meta-analysis of research on same-gender IPV: "there are few studies with sufficient methodological quality published on intimate partner violence in self-identified lesbian couples" (p. 56). The lack of methodological quality and consistency across empirical investigations of IPV among sexual minority women calls into question the utility of theoretical and explanatory models for IPV among heterosexual populations, as well as the measures 
designed to assess the components of such models, in investigating the factors and mechanisms potentially underlying IPV among non-heterosexual populations (Davis \& Glass, 2011; Hassouneh \& Glass, 2008).

Specifically, throughout Part I of this document I named several instances in which sexual minority women's voices were overlooked, silenced, inappropriately spoken for, or misrepresented entirely. In the months since I originally wrote those critiques calling out the prevailing heterosexism in research fields, there has been a substantial increase in the number of published research articles specifically examining causes of IPV among sexual minority women, as well as other sexual minority populations. Importantly, however, a considerable portion of the still growing body of research in this area remains focused on substance abuse and anger as key components underlying sexual minority women's same-gender IPV perpetration. While there is evidence that substance abuse remains an issue facing members of the LGBTQ population, including sexual minority women, this evidence most consistently points to substance abuse as a consequence of the historic and continued marginalization of members of the LGBTQ population (I. H. Meyer, 1995, 2003, 2010).

Conversely, the interplay among identity, gender, and minority stressors, such as that found among experiences shared by participants in this study, remains less commonly examined as a possible key aspect of the underlying root causes of female same-sex IPV perpetration. The present study's findings challenge this focus on substance abuse and anger as primary explanations for IPV among sexual minority women by providing evidence for a much more complex level of experience among members of this population. In particular, this study's findings indicate that sexual minority women's 
experiences with minority stressors are not confined to minority stressors specific to their gender identities and sexual orientations, but in fact intersect with minority stressors related to race and class as well. The complexity of minority stress related experiences shared by participants in this study suggest that practitioners currently working with, or exploring the possibility of working with, LGBTQ women who have perpetrated and/or experienced victimization of intimate partner abuse might seek to acknowledge and integrate this complexity in the services they provide to members of this population.

Specifically, qualitative findings regarding participants' experiences and identities (see RQ1 Findings in Chapter 6 ) indicate that LGBTQ women's experiences with minority stressors are not confined to stressors specific to their gender identities and sexual orientations, but in fact intersect with minority stressors related to race and class as well. Importantly, however, marginalized identity characteristics related to race, economic class, and sexual orientation should not be considered as key risk factors for women's perpetration of same-gender IPV. Rather, experiences of minority stressors related to these identity characteristics might influence the effectiveness of IPV interventions among female perpetrators. IPV intervention and prevention professionals, as well as practitioners working in related fields (e.g., criminal justice, mental health, and public health, etc.), can acknowledge and integrate this complexity in their work by first recognizing the social and cultural contributing factors to the invisibility of sexual minority women in IPV intervention and prevention approaches. For example, while targeting issues of male privilege in IPV perpetrator interventions with men is a reasonable approach back by a considerable body of research evidence regarding men's use of their privilege as both a motivation and tool for their IPV perpetration, the same 
approach is not only likely to be contextually irrelevant to sexual minority women, but also largely inappropriate given the complexities of the overall systemic marginalization experienced by this population. Sexual minority women who perpetrate IPV are also likely to have experiences of victimization in terms of both interpersonal physical violence, and less overt forms of violence and oppression occurring on a more constant, or day-to-day, basis (i.e., microaggressions; I.H. Meyer, 2010; Sue, 2010; Sue et al., 2007). Given the present study's exploratory findings regarding the thematic and conceptual issue of control in sexual minority women's minority stress and gender-related experiences, IPV intervention and prevention practitioners could enhance their work as it relates to this population by encouraging current intervention participants to discuss the influence(s) of minority stress experiences on their interpersonal interactions and relationships. In doing so, the practitioner could maintain a focus on IPV intervention participants' individual accountability for their actions, while also attempting to address these individuals' underlying motivations for perpetrating abuse toward their same gender partner through a more precise approach than that offered by a primary focus on substance abuse.

\section{Overarching Program of Research Next Steps}

Findings from this study's emergent design and systematic analysis will be used in designing and implementing the next set of research phases planned for the overarching program of research in which this study is situated. Specifically, findings from this study, including lessons learned from the design, recruitment, implementation, and data analytic processes will be used to first inform a second series of interviews and focus groups. The intent for this second series of interviews is to focus specifically on recruiting individuals 
with histories of IPV perpetration and victimization to participate in think aloud interviews (Collins, 2003) in which participants will each be asked to provide immediate responses to survey items in the measures evaluated in the present study, as well as measures specifically related to intimate partner violence perpetration and victimization (e.g., the Conflict Tactics Scales; Straus, 1979; Straus et al., 1996). The qualitative findings from this latter investigation combined with those from the present study will be subsequently used in determining and implementing revisions to measures. Subsequent research phases will then utilize the revised measures to determine the relations among gender role adherence, gender role stress, minority stress, social dominance, antifemininity, and the perpetration of female same-sex IPV (see Figure 2). This mixedmethods program of research is conceptualized as an iterative process geared toward elevating the voices of the population of interest (i.e., sexual minority women) in order to further develop practical recommendations for IPV intervention and prevention strategies specific to the needs of this historically marginalized population. 


\section{Tables}

Table 1.

Gender-Relevant and Violence Assessment Measures

\begin{tabular}{|c|c|c|}
\hline Target Construct & Measure $^{\mathrm{a}}$ & $N_{\text {items }}$ \\
\hline \multirow{3}{*}{$\begin{array}{l}\text { GENDER IDENTITY AND GENDER } \\
\text { EXPRESSION }\end{array}$} & 1. Bem Sex Role Inventory (BSRI) & 60 \\
\hline & $\begin{array}{l}\text { 2. Gender Expression Measure among Sexual } \\
\text { Minority Women (GEMSMW) }\end{array}$ & 15 \\
\hline & $\begin{array}{l}\text { 3. *Masculine Gender Identity Scale for Females } \\
\text { (MGISF) }\end{array}$ & 29 \\
\hline \multirow[t]{3}{*}{ GENDER ROLE ADHERENCE } & 4. Femininity Ideology Scale (FIS) & 45 \\
\hline & 5. Male Role Norms Inventory - Revised (MRNIR) & 39 \\
\hline & 6. Social Dominance Orientation Scale (SDO) & 16 \\
\hline \multirow[t]{2}{*}{ GENDER Role STRESS } & 7. Feminine Gender Role Stress Scale (FGRS) & 39 \\
\hline & 8. Masculine Gender Role Stress Scale (MGRS) & 40 \\
\hline \multirow[t]{3}{*}{ MINORITY STRESS } & 9. Lesbian Internalized Homophobia Scale (LIHS) & 52 \\
\hline & 10. Outness Inventory (OI) & 27 \\
\hline & 11. Stigma Consciousness Questionnaire (SCQ) & 20 \\
\hline IPV $^{\mathrm{B}}$ PERPETRATION & $\begin{array}{l}\text { 12. * Revised Conflict Tactics Scales - Perpetration } \\
\text { (CTS-2-P) }\end{array}$ & 39 \\
\hline IPV VICTIMIZATION & $\begin{array}{l}\text { 13. }{ }^{*} \text { Revised Conflict Tactics Scales - } \\
\text { Victimization (CTS-2-V) }\end{array}$ & 40 \\
\hline
\end{tabular}

${ }^{a}$ Measures marked with ${ }^{* \prime}$ were removed from the formal evaluation analysis. 1 . Auster \& Ohm (2000); Bem (1981a); Myers \& Gonda (1982); Holt \& Ellis (1998). 2. Lehavot et al. (2011). 3. Blanchard \& Freund (1983). 4. Levant et al. (2007a). 5. Levant et al. (2010); Levant et al. (2007b); Levant et al. (2013). 6. Ho et al. (2015). 7. Gillespie \& Eisler (1992). 8. Eisler \& Skidmore (1987). 9. Szymanski \& Chung (2008). 10. Mohr \& Fassinger (2000). 11. Pinel (1999). 12. Murray A. Straus (1979); M. A. Straus et al. (1996). 13. Murray A. Straus (1979); M. A. Straus et al. (1996). 
Table 2.

Participant Eligibility Criteria from Initial Focus Groups Design

\begin{tabular}{ll}
\hline Participant Category & \multicolumn{1}{c}{ Eligible Participant Characteristics ${ }^{\text {a }}$} \\
\hline All & Identifies as female and/or goes by her/hers/she pronouns on a regular \\
& basis. \\
1 & SMW - Currently single \\
3 & SMW - In romantic relationship \\
4 & SMW - In romantic relationship - and FSSIPV-P \\
\hline
\end{tabular}

${ }^{a} \boldsymbol{S M W}=$ Sexual minority women $; \boldsymbol{F S S I P V - P}=$ Female same-sex intimate partner violence - perpetration; $\boldsymbol{F S S I P V - V}=$ Female same-sex intimate partner violence - victimization . 
Table 3.

Participant Screening Interview and Post-Interview/Post-Focus Group Survey Questions

\begin{tabular}{|c|c|c|}
\hline Question & Label & Question \\
\hline \multicolumn{3}{|c|}{ SCREENING INTERVIEW } \\
\hline SI-Q.1 & LGBTQ-Identity & $\begin{array}{l}\text { This study is recruiting individuals from the greater Portland } \\
\text { and Seattle areas who identify as sexual minority women (such } \\
\text { as bisexual, lesbian, and queer women). Do you identify as a } \\
\text { sexual minority women? }\end{array}$ \\
\hline SI-Q.2 & Relationship Status & $\begin{array}{l}\text { Are you currently in a romantic relationship (this could include } \\
\text { being in a committed or non-committed monogamous or } \\
\text { polyamorous relationship)? }\end{array}$ \\
\hline SI-Q.3 & $\begin{array}{l}\text { (IF YES TO SQ-2) } \\
\text { Partner LGBTQ- } \\
\text { Identity }\end{array}$ & $\begin{array}{l}\text { Does your primary romantic partner identify as a sexual } \\
\text { minority woman? (i.e., she, her, hers)? }\end{array}$ \\
\hline SI-Q.4 & IPV Victimization-Past & $\begin{array}{l}\text { Have you ever experienced physical, psychological, or } \\
\text { emotional abuse from a former romantic partner? }\end{array}$ \\
\hline SI-Q.5 & & Has a former romantic partner ever stalked you? \\
\hline SI-Q.6 & $\begin{array}{l}\text { (IF YES TO SQ-2) IPV } \\
\text { Victimization-Current }\end{array}$ & $\begin{array}{l}\text { Have you ever experienced physical, psychological, or } \\
\text { emotional abuse from your current romantic partner? }\end{array}$ \\
\hline SI-Q.7 & & Has your current romantic partner ever stalked you? \\
\hline SI-Q.8 & IPV Perpetration-Past & $\begin{array}{l}\text { Have you ever been physically, psychologically, or emotionally } \\
\text { abusive toward a former romantic partner? }\end{array}$ \\
\hline SI-Q.9 & & Have you ever stalked a former romantic partner? \\
\hline SI-Q.10 & $\begin{array}{l}\text { (IF YES TO SQ-2) IPV } \\
\text { Perpetration-Current }\end{array}$ & $\begin{array}{l}\text { Have you ever been physically, psychologically, or emotionally } \\
\text { abusive toward your current romantic partner? }\end{array}$ \\
\hline SI-Q.11 & & Have you ever stalked your current romantic partner? \\
\hline \multicolumn{3}{|c|}{ POST-INTERVIEW/FOCUS GROUP SURVEY } \\
\hline PS-Q.1 & Age & How old are you? \\
\hline PS-Q.2 & Native Language & Is English your first language? \\
\hline PS-Q.3 & Gender Pronoun & $\begin{array}{l}\text { What gender pronoun do you most often go by (e.g., her, hir, } \\
\text { she, ze, etc.) }\end{array}$ \\
\hline PS-Q.4 & $\begin{array}{l}\text { Sexuality/Sexual } \\
\text { Orientation }\end{array}$ & $\begin{array}{l}\text { Which of the following best describes your sexuality? (note: } \\
\text { options are listed in alphabetical order) }\end{array}$ \\
\hline PS-Q.5 & Race/Ethnicity & What is your race/ethnicity? \\
\hline PS-Q.6 & Participation Reason & Why did you decide to participate in today's interview? \\
\hline PS-Q.7 & Series 2 Interest & $\begin{array}{l}\text { Would you be interested in participating in a second one-on-one } \\
\text { interview or a focus group for this study? }\end{array}$ \\
\hline PS-Q.8 & Final Comments & $\begin{array}{l}\text { Do you have any final comments on the topics discussed in } \\
\text { today's interview? }\end{array}$ \\
\hline
\end{tabular}


Table 4.

Sample Characteristics

\begin{tabular}{|c|c|c|}
\hline Descriptive Variable & $\mathbf{N}_{\text {group }}$ & $\%\left(N_{\text {total }}=14\right)$ \\
\hline \multicolumn{3}{|l|}{ PARTICIPATION TYPE } \\
\hline Focus Group & 4 & $29 \%$ \\
\hline One on One & 10 & $71 \%$ \\
\hline \multicolumn{3}{|l|}{ PARTICIPANT CATEGORY } \\
\hline In Relationship/IPV-V & 1 & $7 \%$ \\
\hline In Relationship/IPV-VP & 2 & $14 \%$ \\
\hline In Relationship/No IPV & 6 & $43 \%$ \\
\hline Single/IPV-V & 1 & $7 \%$ \\
\hline Single/IPV-VP & 3 & $21 \%$ \\
\hline Single/No IPV & 1 & $7 \%$ \\
\hline \multicolumn{3}{|l|}{ IPV HISTORY } \\
\hline NO IPV-V & 6 & $43 \%$ \\
\hline YES IPV-V & 8 & $57 \%$ \\
\hline NO IPV-P & 12 & $86 \%$ \\
\hline YES IPV-P & 2 & $14 \%$ \\
\hline \multicolumn{3}{|l|}{ RELATIONSHIP STATUS } \\
\hline In Relationship & 10 & $71 \%$ \\
\hline Single & 4 & $29 \%$ \\
\hline \multicolumn{3}{|l|}{ RACE } \\
\hline Multiracial & 6 & $43 \%$ \\
\hline White & 8 & $57 \%$ \\
\hline \multicolumn{3}{|l|}{ RACE - MULTIRACIAL IDENTITY } \\
\hline Black/Indigenous/Hullah & 1 & $7 \%$ \\
\hline Black/Multiracial & 1 & $7 \%$ \\
\hline Mestiza & 1 & $7 \%$ \\
\hline Mixed & 1 & $7 \%$ \\
\hline Non-White/Native American/Middle-Eastern & 1 & $7 \%$ \\
\hline White/Alaska-Native & 1 & $7 \%$ \\
\hline \multicolumn{3}{|l|}{ PREFERRED PRONOUNS } \\
\hline Her/Hers/She & 11 & $79 \%$ \\
\hline She or They & 2 & $14 \%$ \\
\hline They/Them/Theirs & 1 & $7 \%$ \\
\hline \multicolumn{3}{|l|}{ SEXUAL ORIENTATION } \\
\hline Bisexual & 4 & $29 \%$ \\
\hline Gay & 1 & $7 \%$ \\
\hline Lesbian & 5 & $36 \%$ \\
\hline Queer & 3 & $21 \%$ \\
\hline Other & 1 & $7 \%$ \\
\hline
\end{tabular}

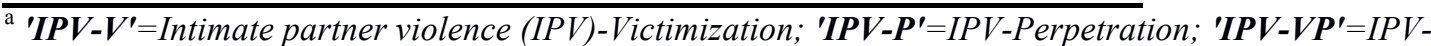
Victimization \& IPV-Perpetration. 
Table 5.

Sample Excerpt of Coded Interview Data Segment.

\begin{tabular}{|c|c|}
\hline Excerpt & Coding $^{b}$ \\
\hline $\begin{array}{l}\text { P4 }^{\text {a }} \text { : [note] I think... I think I've been lucky I } \\
\text { feel like I've been lucky to be able to come } \\
\text { out of it and then yeah be confident and find } \\
\text { myself and be happy and find a place where I } \\
\text { do feel safe 'cause I know not everyone is as } \\
\text { fortunate. Um for those that are brave enough } \\
\text { to try to pursue their own identity and really } \\
\text { be who they are um in certain places it's just } \\
\text { not [BRIEF PAUSE] they might not have } \\
\text { the- the same support systems or the same } \\
\text { options that I had in order to get myself to a } \\
\text { safer place where I can really [I: yeah] be } \\
\text { myself. Umm... so I mean I'm glad I went } \\
\text { through it 'cause I feel like it's made me a } \\
\text { stronger person and more compassionate to } \\
\text { others maybe going through the same thing. } \\
\text { Um but it was hard it still hurts sometimes } \\
\text { [BOTH CHUCKLE] to think about things } \\
\text { that I've gone through and how some like I } \\
\text { mentioned some things haven't changed } \\
\text { some things are maybe getting worse [BOTH } \\
\text { LAUGH] for certain people but overall just } \\
\text { yeah trying to stay 'posi' and [BOTH } \\
\text { LAUGH] get ahead yeah. 'Cause things can } \\
\text { get better yeah. }\end{array}$ & $\begin{array}{l}\text { C4 }{ }^{\mathrm{c}} \text { [SURVIVAL] I've been lucky to come out of it } \\
\text { C4_I am confident } \\
\text { C4_I have been able to find myself } \\
\text { C4_I have been able to be happy } \\
\text { C4_I have been able to find a place where I feel safe } \\
\text { C4_Pursuing your own identity takes bravery } \\
\text { C4_Being who you are takes bravery } \\
\text { C4_[PLACE] Not every place is safe for everyone to } \\
\text { be who they are } \\
\text { C4_[SociAL SuPPORT] Not everyone has a support } \\
\text { system to be able to safely be themselves } \\
\text { C4_Not everyone has the option to pursue their own } \\
\text { identity } \\
\text { C4_Not everyone has the option to be themselves } \\
\text { C4_I'm glad I went through it } \\
\text { C4_Pursuing my own identity made me a stronger } \\
\text { person } \\
\text { C4_Pursuing my own identity made me more } \\
\text { compassionate to others similar to me } \\
\text { C4_It was hard } \\
\text { C4_It still hurts sometimes } \\
\text { C4_[SociAL CHANGE] Some things have not changed } \\
\text { C4_[SoCIAL CHANGE] Some things are getting worse } \\
\text { for certain ppl } \\
\text { C4_Staying positive helped me get through it }\end{array}$ \\
\hline
\end{tabular}

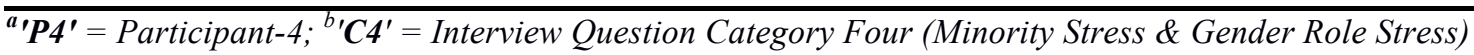


Table 6.

Kappa Statistics per Measure

\begin{tabular}{lll}
\hline Category & Measure $^{\text {a }}$ & kappa \\
\hline ALL CATEGORIES & All Measures & 0.54 \\
GENDER EXPRESSION & BSRI & 0.49 \\
& GEMSMW & 0.42 \\
GENDER ROLE ADHERENCE & FIS & 0.67 \\
& MRNIR & 0.41 \\
& SDO & 0.39 \\
GENDER ROLE STRESS & FGRS & 0.69 \\
& MGRS & 0.46 \\
MINORITY STRESS & LIHS & 0.48 \\
& OI & 0.48 \\
& SCQ & 0.50 \\
\hline
\end{tabular}

${ }^{a}$ BSRI $=$ Bem Sex Role Inventory (Auster \& Ohm, 2000; Bem, 1981a); GEMSMW = the Gender Expression Measure among Sexual Minority Women (Lehavot et al., 2011), FIS = the Femininity Ideology Scale (Levant et al., 2007), MRNIR = the Male Role Norms Inventory Revised (Levant et al., 2013, 2010, 2007b), SDO = the Social Dominance Orientation Scale (Ho et al., 2015), FGRS = the Feminine Gender Role Stress Scale (Gillespie \& Eisler, 1992), MGRS = the Masculine Gender Role Stress Scale (Eisler \& Skidmore, 1987), LIHS = the Lesbian Internalized Homophobia Scale (Szymanski \& Chung, 2008), OI = the Outness Inventory (Mohr $\&$ Fassinger, 2000), and $\boldsymbol{S C Q}=$ the Stigma Consciousness Questionnaire (Pinel, 1999). 
Table 7.

Codes with Disagreements Across All Measures

\begin{tabular}{ll}
\hline Codename & Disagreement Frequency \\
\hline ACCEPTANCE & 1 \\
AFFECT & 4 \\
APPEARANCE & 2 \\
BINARY & 2 \\
CHANGE & 3 \\
CHILDHOOD & 1 \\
CHOICE & 1 \\
COMMUNITY & 2 \\
COMPLIANCE & 1 \\
CONFIDENCE & 1 \\
CONFUSION & 1 \\
CONSISTENCY & 1 \\
DOMINANT CULTURE & 4 \\
EQUITABLE ROLES & 1 \\
EXPRESSION & 5 \\
FAMILY & 1 \\
FEMALE & 1 \\
FEMININITY & 3 \\
FEMINISM & 1 \\
GENDER & 3 \\
GENDER EXPRESSION & 3 \\
GENDER ROLE ADHERENCE & 3 \\
GENDER ROLE ENFORCEMENT & 3 \\
GENDER ROLE STRESS & 3 \\
GENDER ROLES & 1 \\
GENDERED CLOTHING & 1 \\
HETERONORMATIVITY & 6 \\
IDENTITY & 2 \\
IDENTITY FIT & 4 \\
IDENTITY WORK & 1 \\
INCLUSION & 2 \\
INDEPENDENCE & 2 \\
INTELLECT & 1 \\
INTERSECTIONALITY & 1 \\
INTERSECTIONALITY-X & 1 \\
LGBTQ COMMUNITY & 1 \\
LOOKING THE PART & 4 \\
MASCULINITY & 2 \\
MEDIA & 1 \\
MENTAL HEALTH & 1 \\
MINORITY STRESS & 1 \\
NATURE OVER NURTURE & 3 \\
OTHERS EXPECTATIONS & 4 \\
OTHERS REACTIONS & 2 \\
PARENTING & 1 \\
PERFORMANCE & \\
PRESENTATION & PRESSURE \\
\hline
\end{tabular}




\begin{tabular}{ll}
\hline PRIVILEGE & 1 \\
ROLES & 3 \\
ROMANTIC LIFE & 1 \\
SELF-PERCEPTION & 1 \\
SELF-PRESENTATION & 2 \\
SEXUAL ORIENTATION & 1 \\
SEXUALITY & 1 \\
SOCIAL CHANGE & 1 \\
SOCIAL LIFE & 5 \\
SOCIAL SUPPORT & 5 \\
STEREOTYPING & 1 \\
SURVIVAL & 4 \\
TITLE & 2 \\
WITHIN GROUP STEREOTYPING & 2 \\
WOMAN & 1 \\
\hline
\end{tabular}


Table 8.

Codebook Coverage per Measurement Category and per Measure

\begin{tabular}{lllll}
\hline Measurement Category & Measure $^{\text {a }}$ & $\boldsymbol{N}_{\text {Items }}$ & $\boldsymbol{\Sigma}_{\text {Codes }}{ }^{\mathbf{b}}$ & $\%_{\text {codebook }}$ \\
\hline GENDER EXPRESSION & & 75 & 15 & $15 \%$ \\
& BSRI & 60 & 12 & \\
& GEMSMW & 15 & 4 & \\
GENDER ROLE ADHERENCE & & 99 & 20 & $19 \%$ \\
& FIS & 44 & 18 & \\
& MRNIR & 39 & 8 & \\
& SDO & 16 & 1 & \\
GENDER ROLE STRESS & & & & \\
& & 79 & 15 & $15 \%$ \\
& FGRS & 39 & 13 & \\
MINORITY STRESS & MGRS & 40 & 10 & \\
& & & & \\
& & 99 & 25 & $24 \%$ \\
& LIHS & 52 & 19 & \\
& OI & 27 & 9 & \\
& SCQ & 20 & 6 & \\
\hline
\end{tabular}

${ }^{a}$ BSRI = Bem Sex Role Inventory (Auster \& Ohm, 2000; Bem, 1981a); GEMSMW $=$ the Gender Expression Measure among Sexual Minority Women (Lehavot et al., 2011), FIS = the Femininity Ideology Scale (Levant et al., 2007), MRNIR = the Male Role Norms Inventory - Revised (Levant et al., 2013, 2010, 2007b), SDO = the Social Dominance Orientation Scale (Ho et al., 2015), $\boldsymbol{F G R S}=$ the Feminine Gender Role Stress Scale (Gillespie \& Eisler, 1992), MGRS = the Masculine Gender Role Stress Scale (Eisler \& Skidmore, 1987), LIHS = the Lesbian Internalized Homophobia Scale (Szymanski \& Chung, 2008), OI = the Outness Inventory (Mohr $\&$ Fassinger, 2000), and $\boldsymbol{S C Q}=$ the Stigma Consciousness Questionnaire (Pinel, 1999). ${ }^{b}$ Reflects the sum of unique codes applied to each measure and measurement' category. 
Table 9.

Codes not Covered by Any of the Evaluated Measures $\left(N_{\text {NotCoded }}=56\right)$

\begin{tabular}{|c|c|}
\hline Construct Category & Codename \\
\hline GENDER & $\begin{array}{l}\text { ANDROGYNY } \\
\text { CIS-GENDER } \\
\text { CONSISTENCY } \\
\text { CULTURE } \\
\text { DOMINANT CULTURE } \\
\text { EQUITABLE ROLES } \\
\text { FLUIDITY } \\
\text { GENDER ROLE STRESS } \\
\text { GENDERED LANGUAGE } \\
\text { MEDIA-ONLINE FORUMS } \\
\text { NATURE OVER NURTURE } \\
\text { PRESENTATION } \\
\text { TWO-SPIRIT }\end{array}$ \\
\hline IDENTITY & $\begin{array}{l}\text { COMMUNITY } \\
\text { EXTERNAL INFLUENCE } \\
\text { MEDIA INFLUENCE } \\
\text { OTHERS CONFUSION } \\
\text { PRIORITY IDENTITY } \\
\text { RACE } \\
\text { SELF-PERCEPTION } \\
\text { SELF-PRESENTATION } \\
\text { SOCIAL MEDIA } \\
\text { TITLE }\end{array}$ \\
\hline MINORITY STRESS & $\begin{array}{l}\text { ADULTHOOD } \\
\text { DAY-TO-DAY } \\
\text { EDUCATING OTHERS } \\
\text { GENDERED CLOTHING } \\
\text { HETERONORMATIVITY } \\
\text { HYPERSEXUALIZED } \\
\text { IDENTITY WORK } \\
\text { IPV } \\
\text { PRIVILEGE } \\
\text { SCHOOL EXPERIENCES } \\
\text { SPACE } \\
\text { SURVIVAL } \\
\text { WITHIN GROUP OPPRESSION } \\
\text { WITHIN GROUP STEREOTYPING }\end{array}$ \\
\hline MINORITY STRESS X GENDER & $\begin{array}{l}\text { BINARY } \\
\text { MISGENDERING } \\
\text { SOCIAL CHANGE }\end{array}$ \\
\hline MINORITY STRESS X IDENTITY & $\begin{array}{l}\text { GENDER EXPRESSION } \\
\text { LOOKING THE PART } \\
\text { MENTAL HEALTH } \\
\text { PRESSURE }\end{array}$ \\
\hline
\end{tabular}




\begin{tabular}{ll}
\hline & RACE IDENTITY \\
MINORITY STRESS $\boldsymbol{X}$ IDENTITY $\boldsymbol{X}$ GENDER & AFFECT \\
& CONTEXT DEPENDENT \\
& GENDER IDENTITY \\
& GENDER ROLE ADHERENCE \\
& IDENTITY FIT \\
& INTERSECTIONALITY-X \\
& PERFORMANCE \\
& PLACE \\
\hline
\end{tabular}


Figures

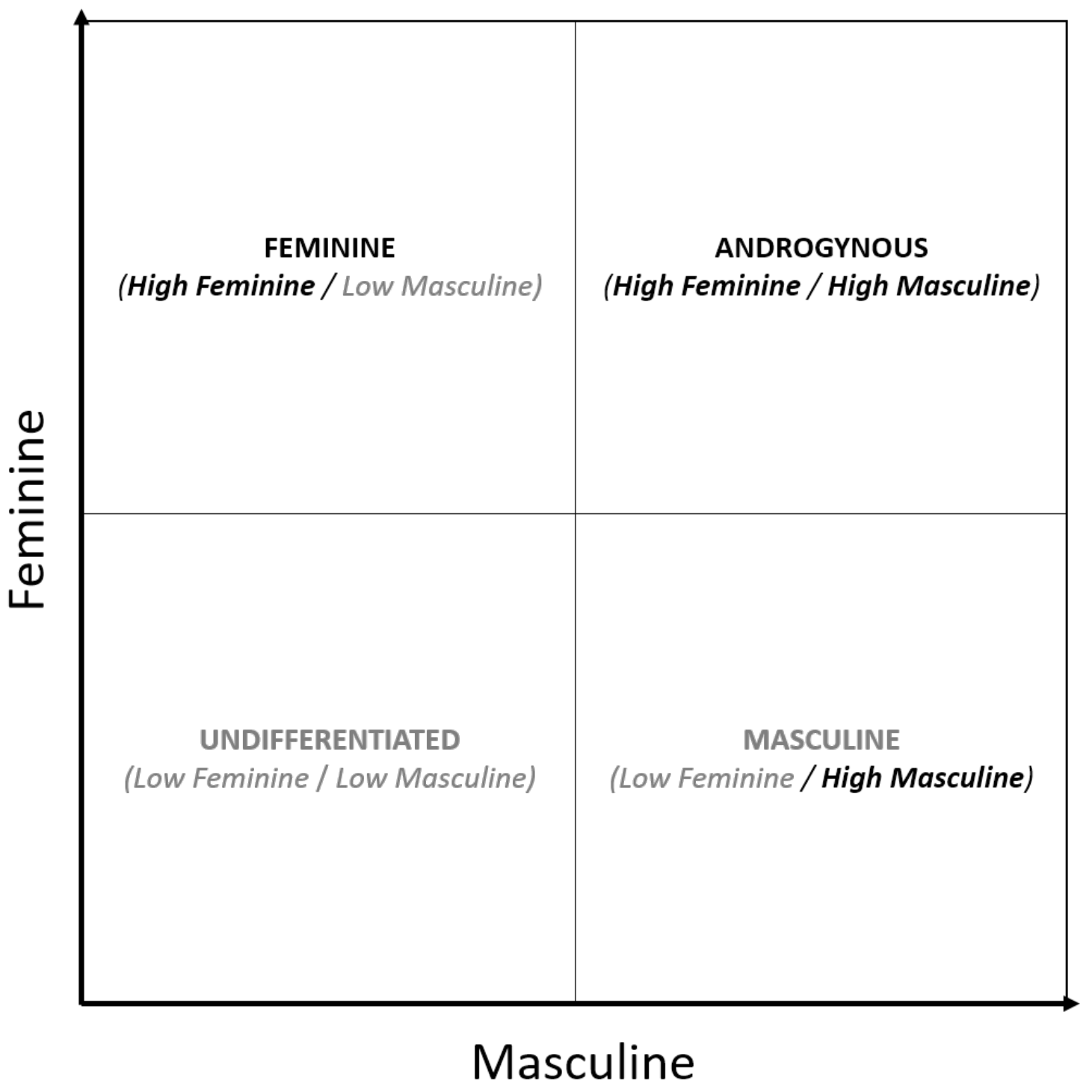

Figure 1. Matrix Visualization of Bem (1981b)'s multi-dimensional gender framework. 


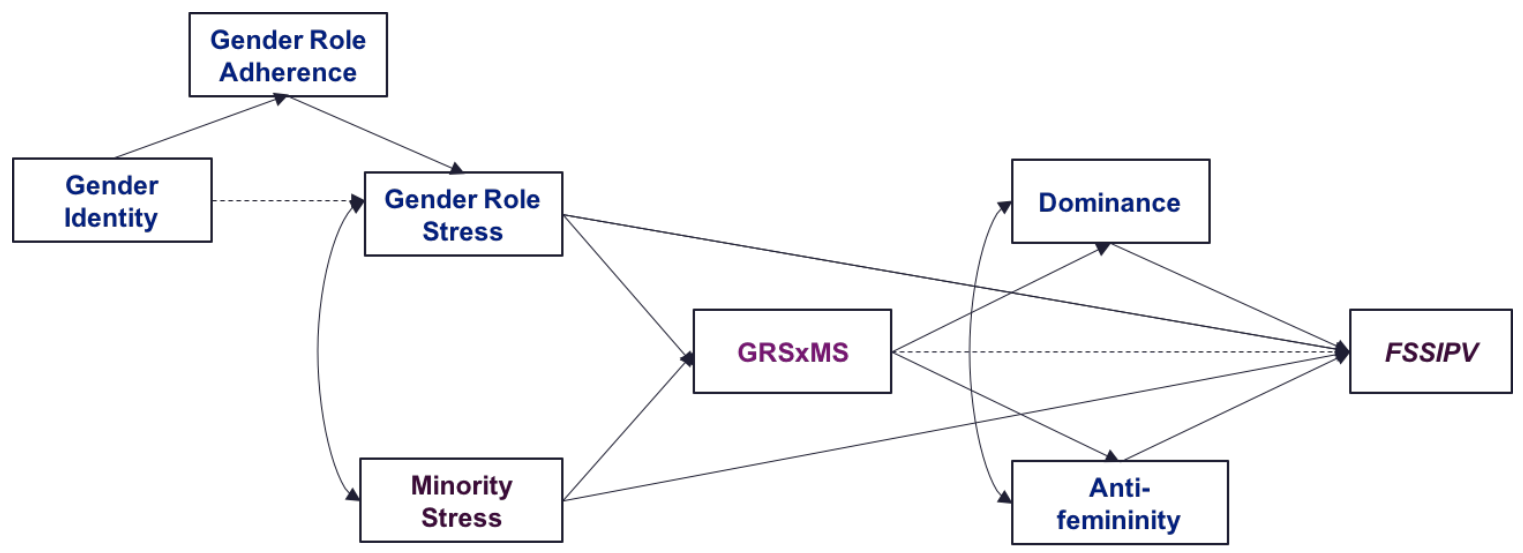

Figure 2. A Working Intersectional Model Predicting Female Same-Sex IPV (FSSIPV). 

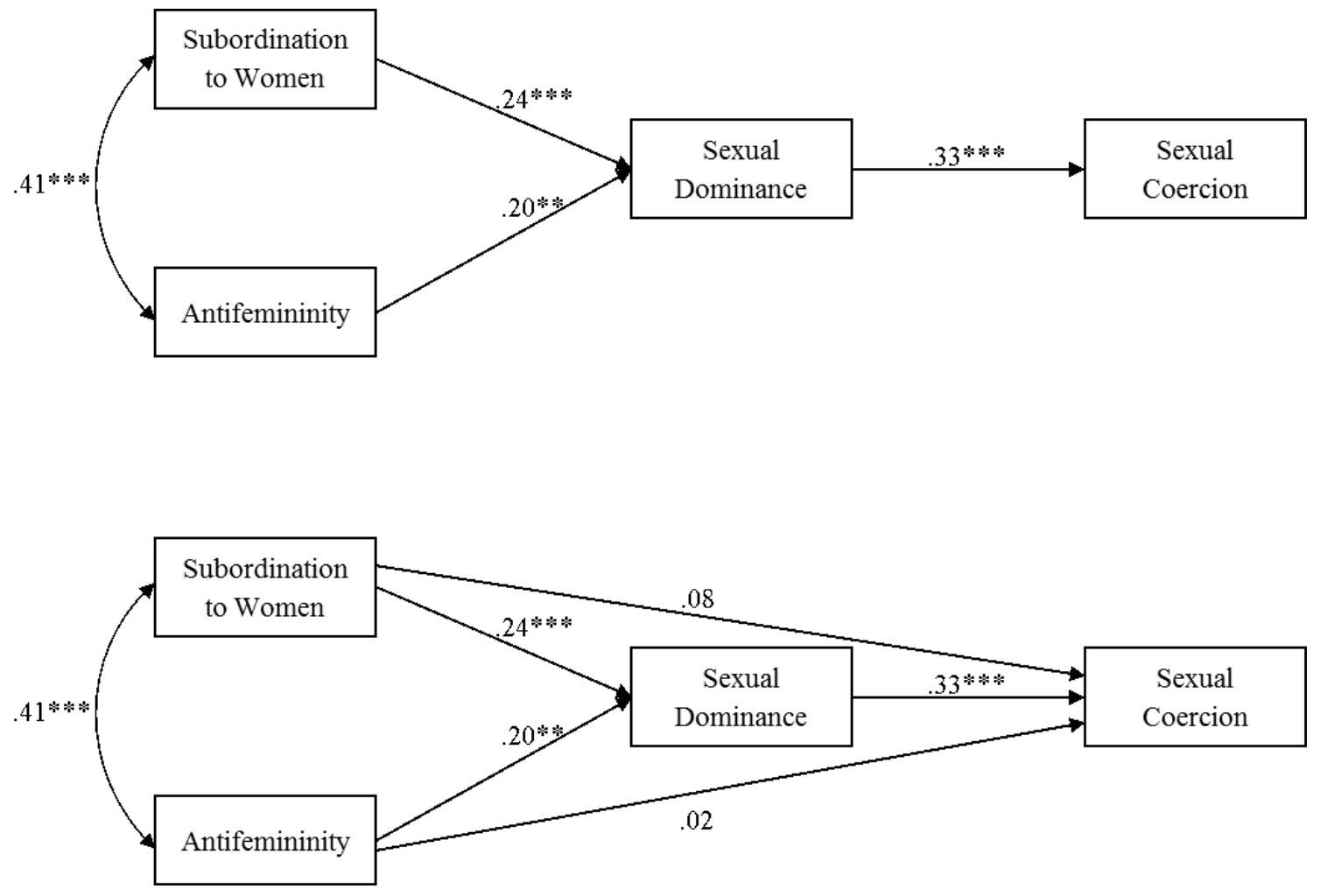

Figure 3. R. M. Smith et al. (2015)'s Mediation models of antifemininity, sexual dominance, and stress associated with subordination to women on sexual coercion. The top panel shows the final mediation model after trimming non-significant paths. The bottom panel shows the full mediation model. 


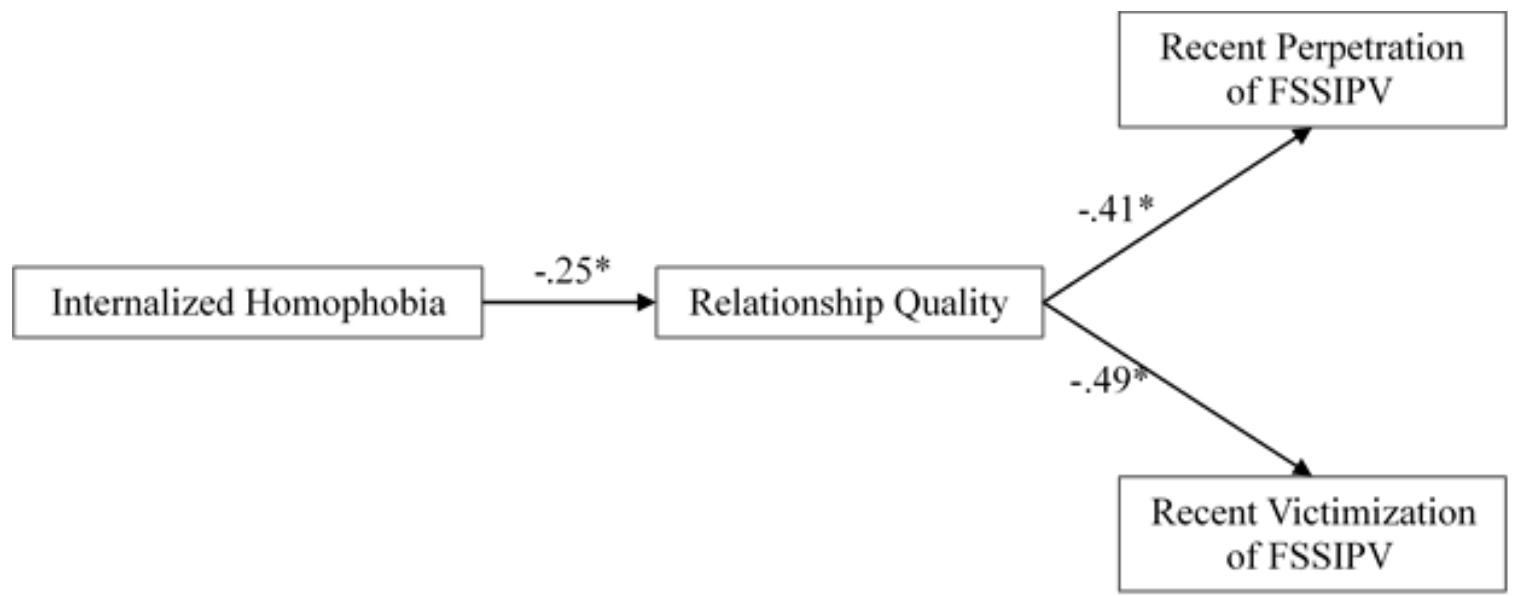

Figure 4. Balsam \& Szymanski (2005)'s Mediational Model Predicting Female Same-Sex IPV Perpetration and Victimization with Supportive Path Analysis Statistics. 


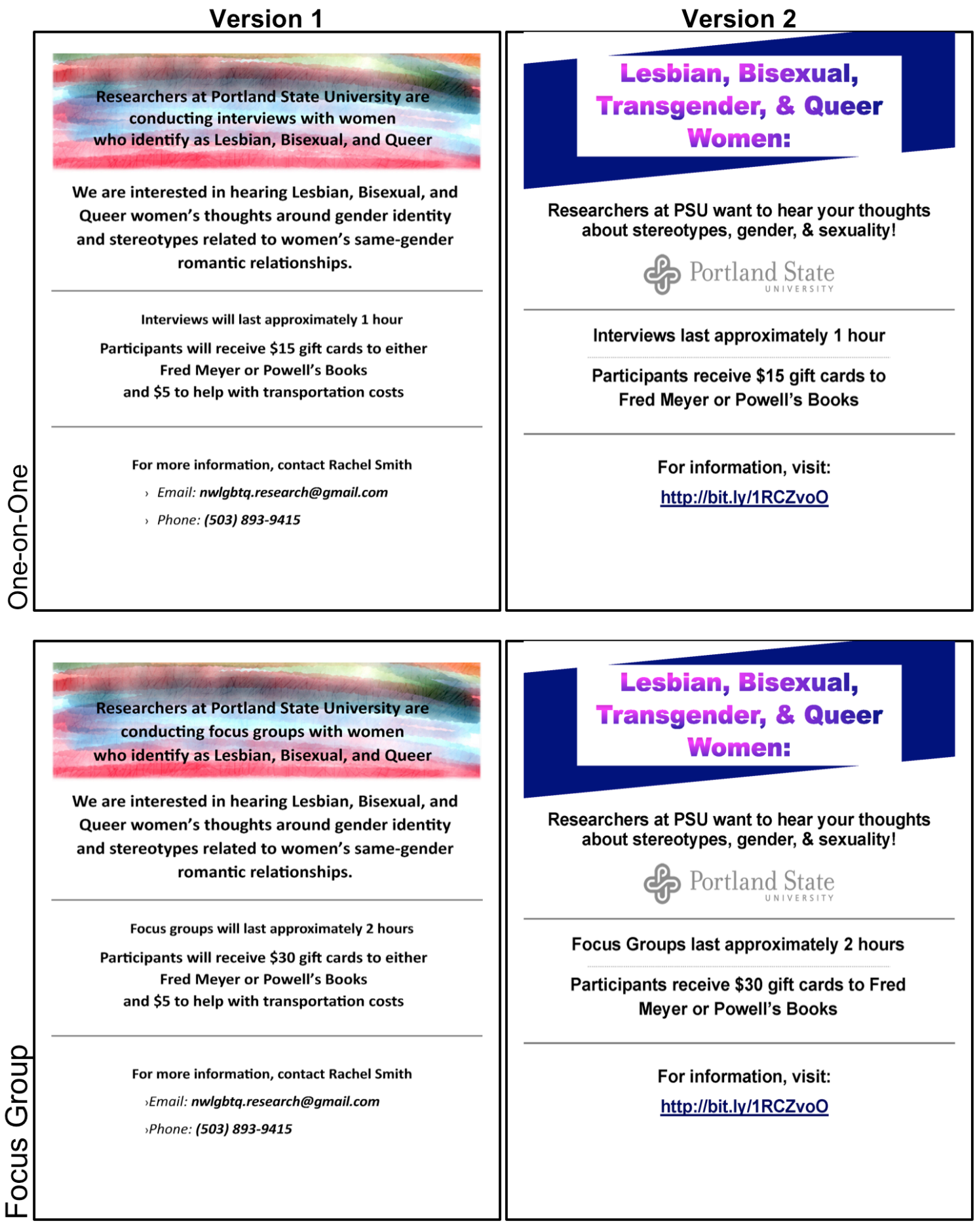

Figure 5. Recruitment Flyers. Detachable tabs containing the contact information from the flyer were added to any printed copies of both Version 1 and Version 2 prior to posting. 


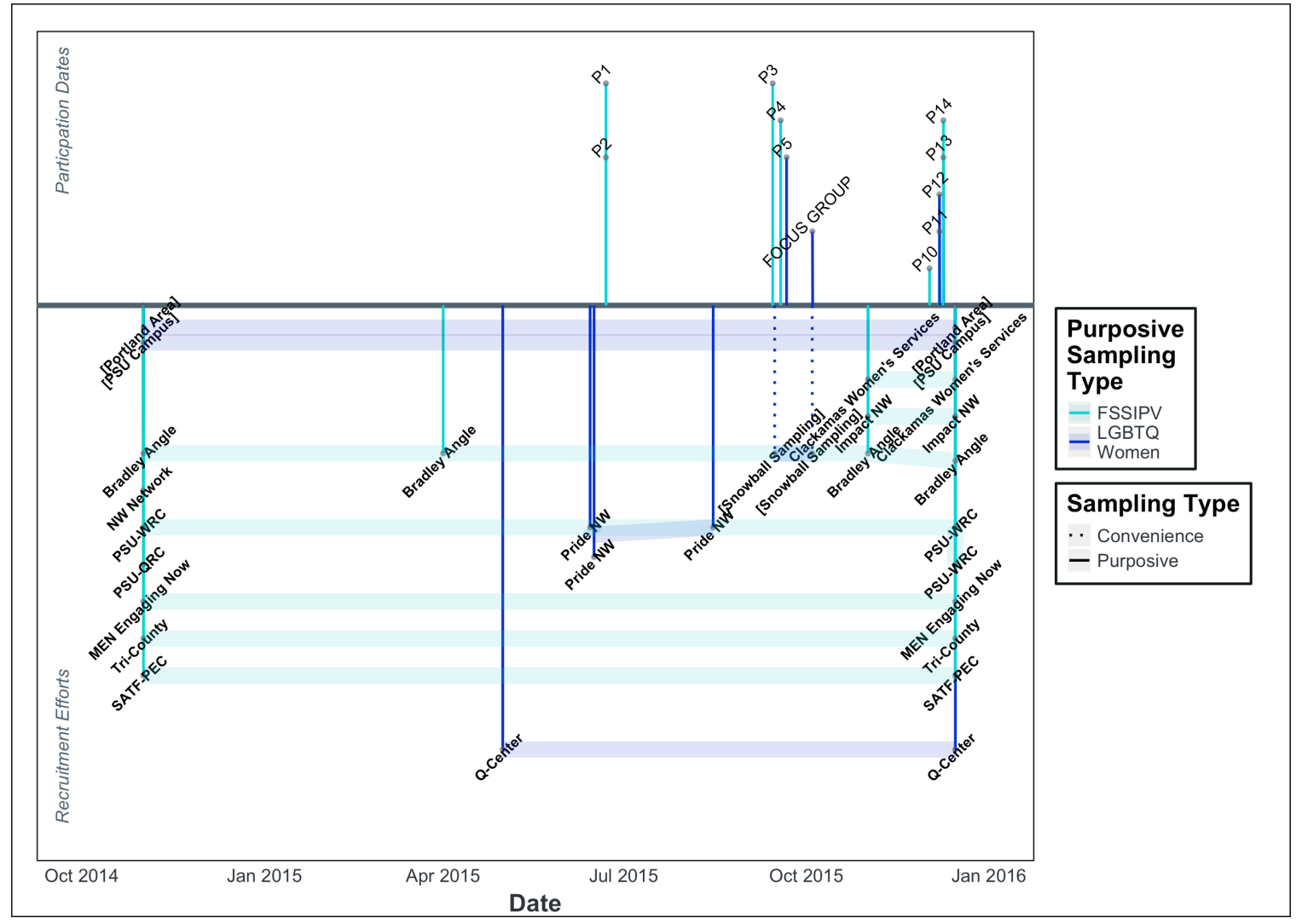

Figure 6. Timeline of Participation Dates and Recruitment Efforts. The top half displays participation dates, with one-on-one interview participants represented as 'P\#' and the four focus group participants represented as 'Focus Group'. The bottom half displays recruitment efforts over the course of the fourteen months of active participant recruitment and data collection. Recruitment efforts are represented by the community partners assisting with participant recruitment: 'PSU' = Portland State University; 'PSU-WRC' = PSU-Women's Resource Center; 'PSU-QRC' = Queer Resource Center; 'SATF-PEC' = Oregon Attorney General's Sexual Assault Task Force's Prevention \& Education Subcommittee. 


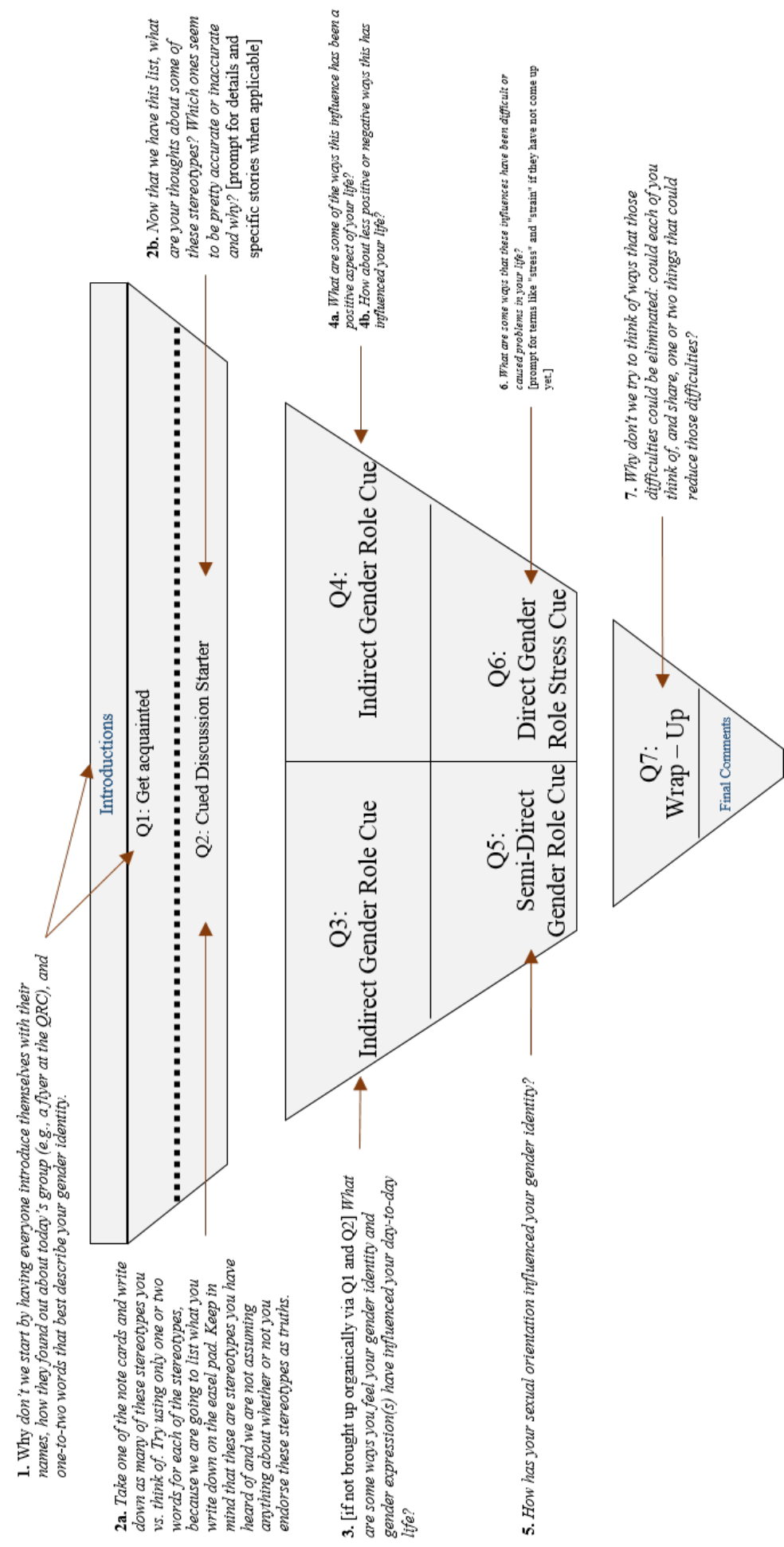

Figure 7. Reverse-Funnel Design (Morgan, 1996b) for Interview and Focus Group Guides. 


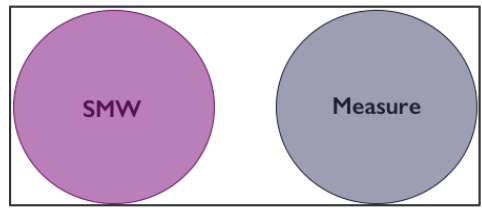

Panel One

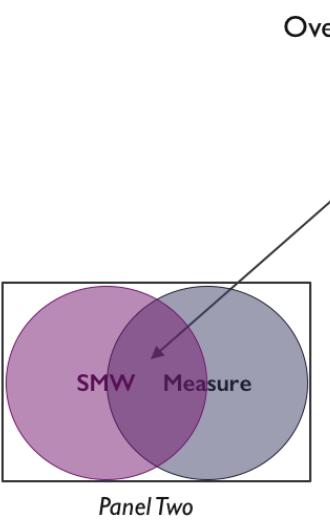

Panel Two
Overlap among Measure and SMW

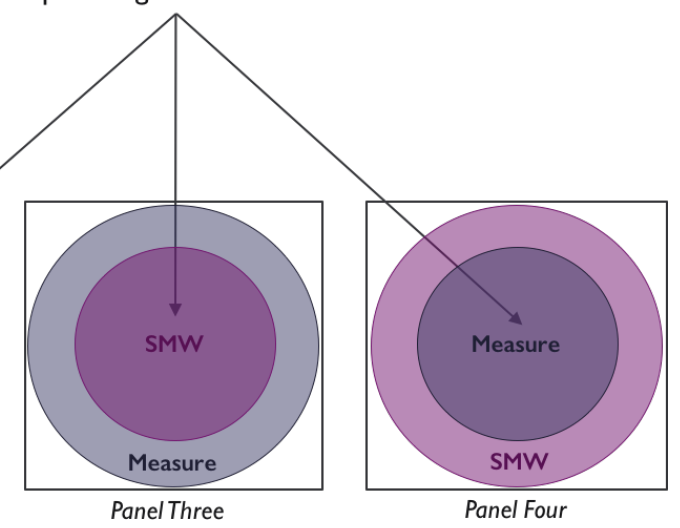

Figure 8. Framework for evaluating overlap and disconnect among measurement tools and IPV-relevant conceptual models. 'SMW' = Sexual minority women's experiences and identities. 'Measure' = The content present in a survey measure, including the language of the survey, experiences and situations described in the survey, and the construct(s), identity(ies), and/or mechanism(s) the survey is intended to measure. 
Gender Expression (GREX) Measures

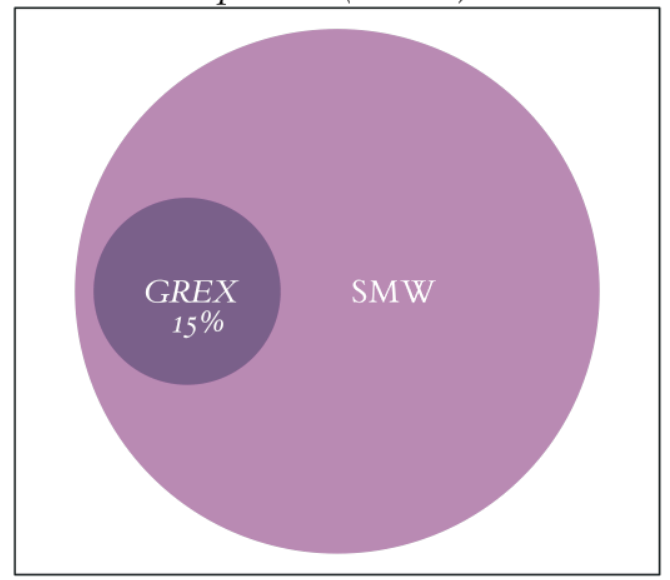

Gender Role Stress (GRS) Measures

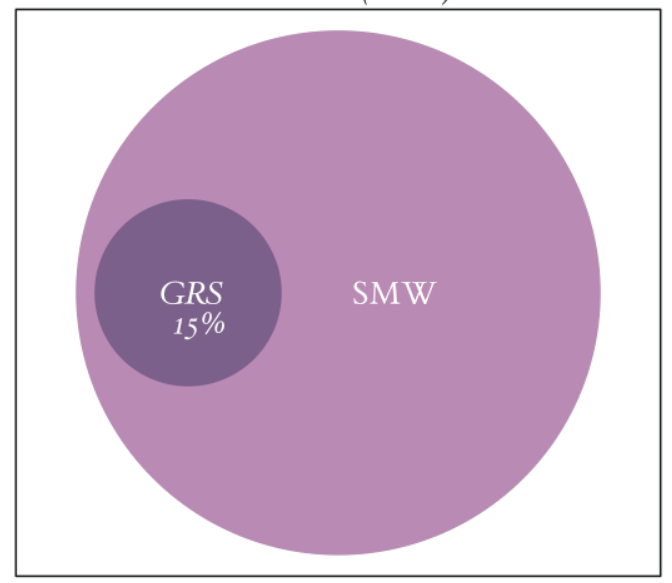

Gender Role Adherence (GRA) Measures

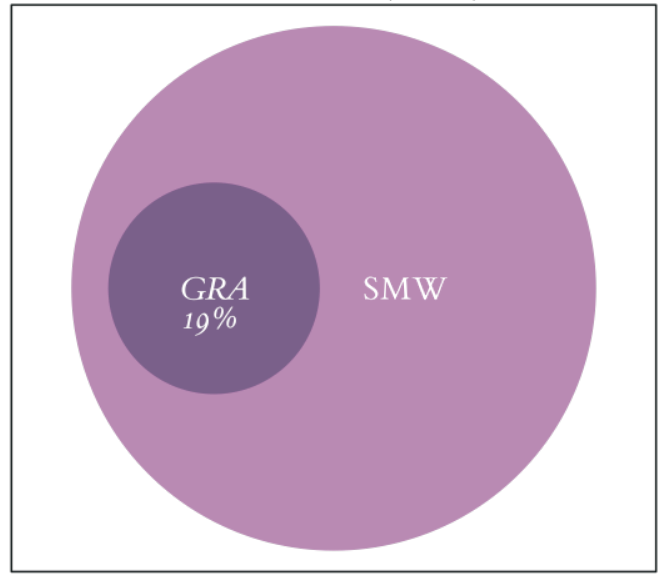

Minority Stress (MS) Measures

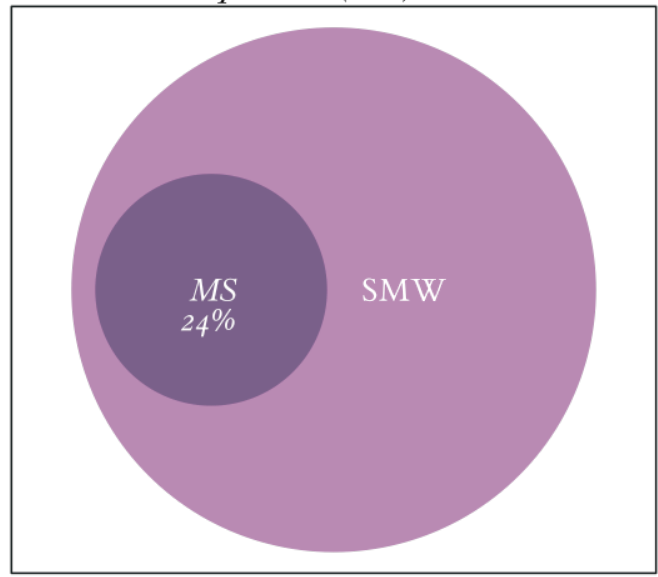

Figure 9. Combined Overlap among Codebook and Each Measurement Category. 'SMW' $=$ The inductively-derived codebook representing the experiences and identities of this study's sample of sexual minority women. Gender expression (GREX) measures include the Bem Sex Role Inventory (Auster \& Ohm, 2000; Bem, 1981a) and the Gender Expression Measure among Sexual Minority Women (Lehavot et al., 2011). Gender role adherence (GRA) measures include the Femininity Ideology Scale (Levant et al., 2007), the Male Role Norms Inventory - Revised (Levant et al., 2013, 2010, 2007b), and the Social Dominance Orientation Scale (Ho et al., 2015). Gender role stress (GRS) measures include the Feminine Gender Role Stress Scale (Gillespie \& Eisler, 1992) and the Masculine Gender Role Stress Scale (Eisler \& Skidmore, 1987). Minority stress (MS) measures include the Lesbian Internalized Homophobia Scale (Szymanski \& Chung, 2008), the Outness Inventory (Mohr \& Fassinger, 2000), and the Stigma Consciousness Questionnaire (Pinel, 1999). 


\section{Combined Overlap among Codebook and All Evaluated Measures}

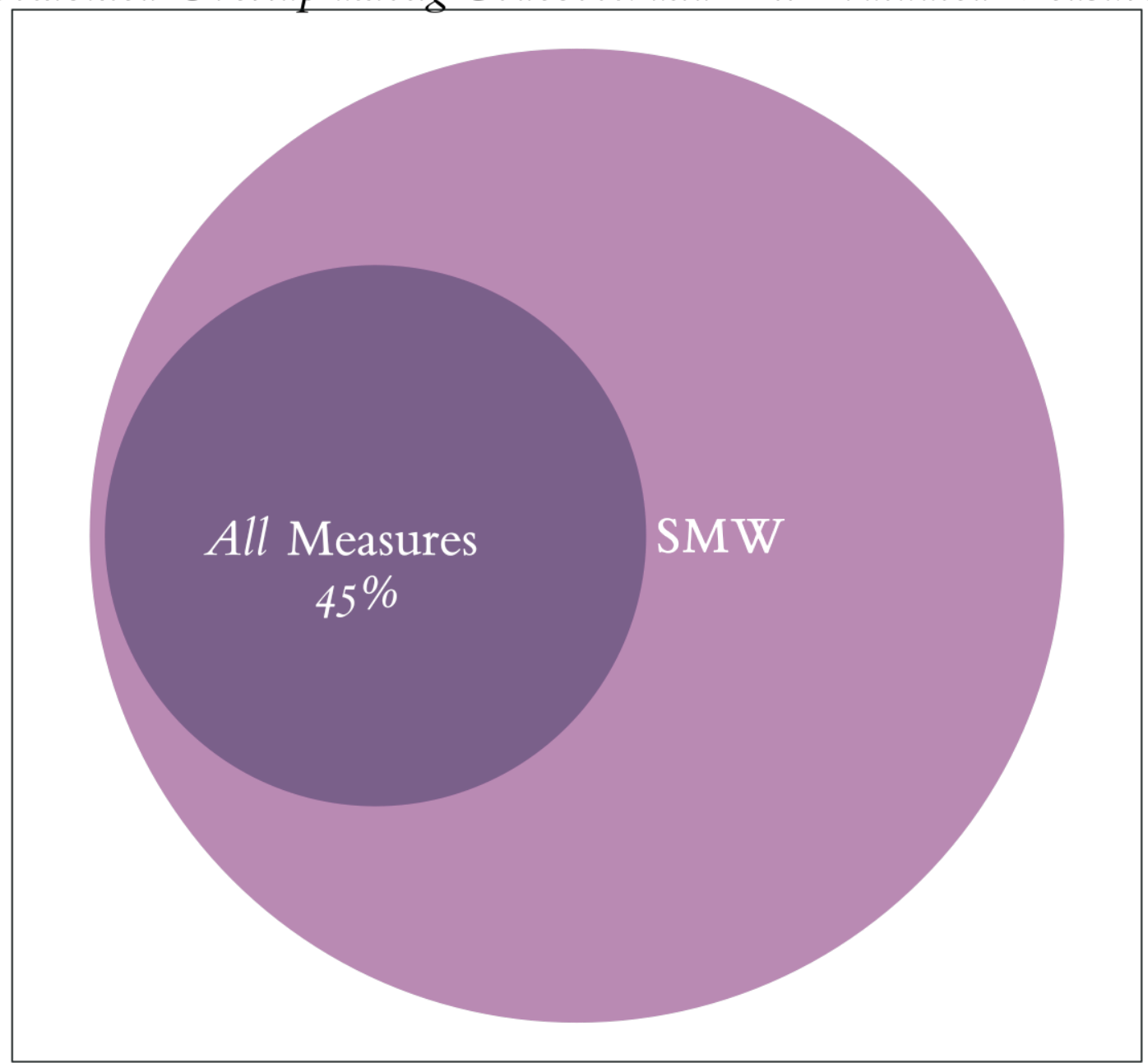

Figure 10. Combined Overlap among Codebook and All Evaluated Measures. 'SMW' = The inductively-derived codebook representing the experiences and identities of this study's sample of sexual minority women. Measures include the Bem Sex Role Inventory (Auster \& Ohm, 2000; Bem, 1981a) Gender Expression Measure among Sexual Minority Women (Lehavot et al., 2011), Femininity Ideology Scale (Levant et al., 2007), Male Role Norms Inventory - Revised (Levant et al., 2013, 2010, 2007b), Social Dominance Orientation Scale (Ho et al., 2015); the Feminine Gender Role Stress Scale (Gillespie \& Eisler, 1992) and the Masculine Gender Role Stress Scale (Eisler \& Skidmore, 1987); the Lesbian Internalized Homophobia Scale (Szymanski \& Chung, 2008), Outness Inventory (Mohr \& Fassinger, 2000), and the Stigma Consciousness Questionnaire (Pinel, 1999). 


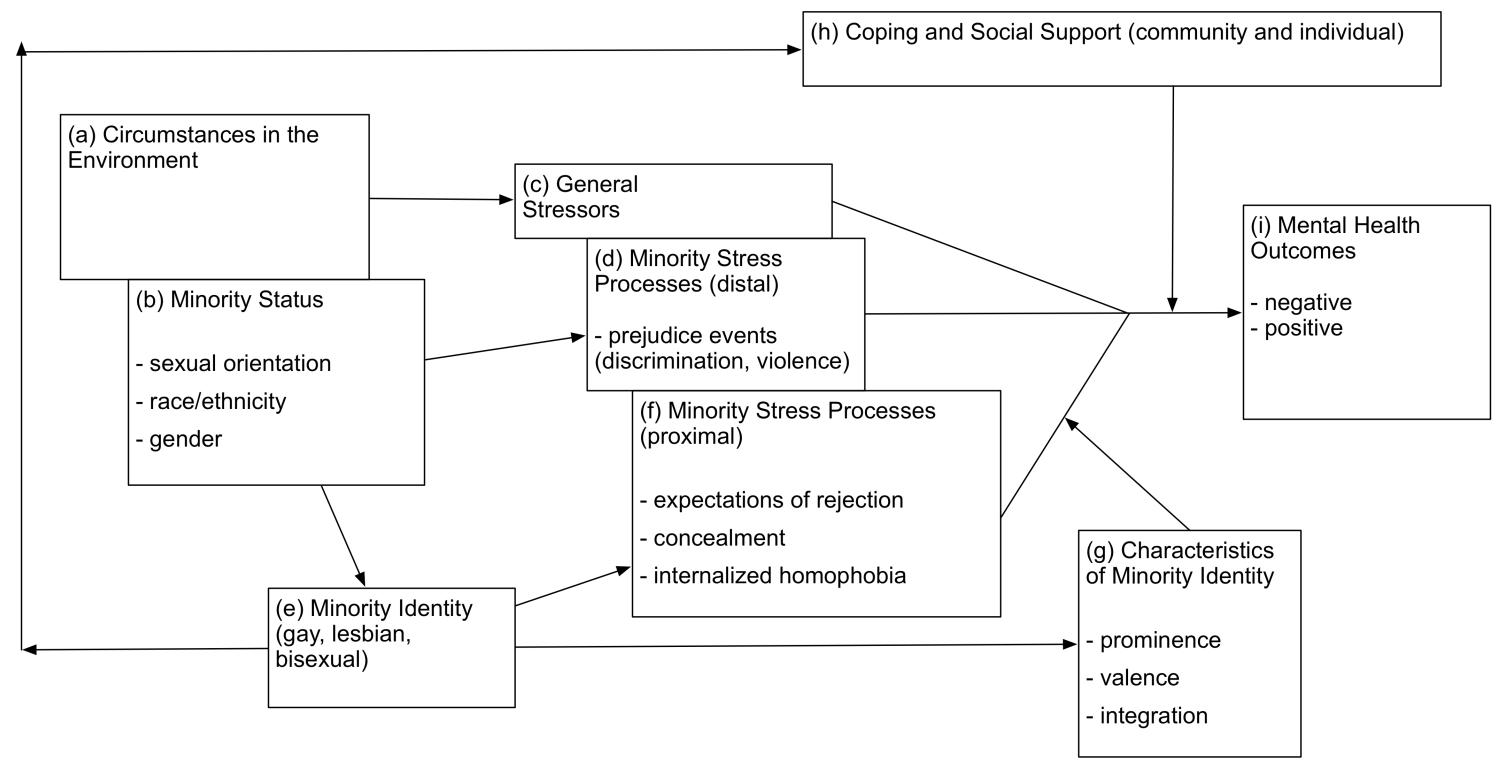

Figure 11. I. H. Meyer (2003)'s Figure 1: "Minority Stress Processes in lesbian, gay, and bisexual populations" (p. 35). 


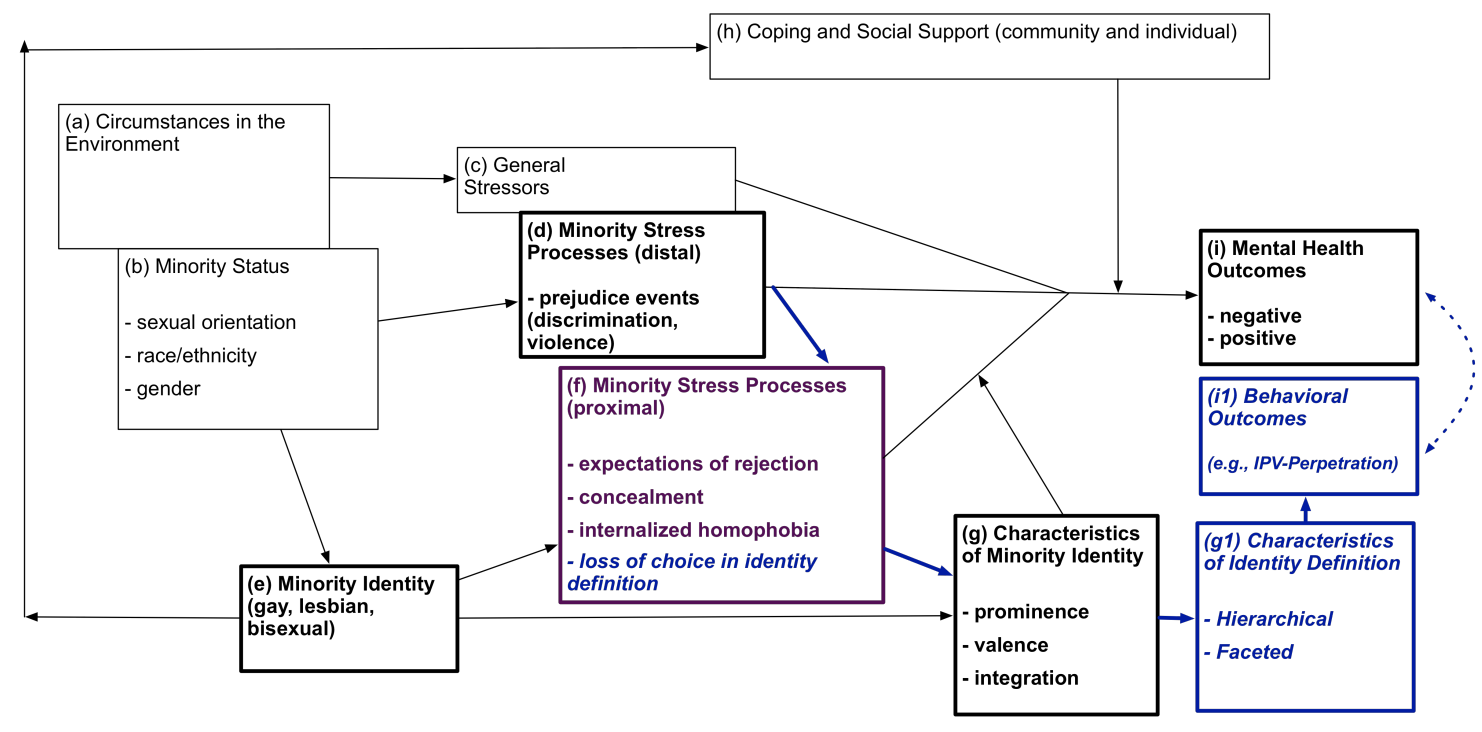

Figure 12. I. H. Meyer (2003)'s conceptual model of minority stress (MS; see I. H. Meyer, 2003, Figure 1) with proposed additions based on the amended minority stress construct definition: "Minority stress as a construct within the modified intersectional model is defined as the loss or diminishing of choice regarding how an individual selfdefines and expresses their identity, or facets of their identity, due to having one or multiple marginalized identities. Minority stress is further conceptualized as a construct existing within the higher-level category of intersectionality. The potential additions to I. H. Meyer (2003)'s minority stress theoretical model are reflected in bold-italicized fonts and enlarged arrows, with bold-non-italicized fonts representing focal areas in I. $H$. Meyer (2003)'s original model. 


\section{References $^{1}$}

Allaire, J., Cheng, J., Xie, Y., McPherson, J., Chang, W., Allen, J., ... Hyndman, R.

(2016). Rmarkdown: Dynamic documents for R. Retrieved from Allison, K. W.

(1998). Stress and oppressed social category membership. In K. Swim \& C.

Stangor (Eds.), Prejudice: The target's perspective (pp. 145-170). San Diego,

CA, US: Academic Press.

Aquino, J. (2016). Descr: Descriptive statistics. Retrieved from

Arnold, J. B. (2016). Ggthemes: Extra themes, scales and geoms for ggplot2. Retrieved

from Ault, A., \& Brzuzy, S. (2009). Removing gender identity disorder from the diagnostic and statistical manual of mental disorders: A call for action. Social Work, 54, 187.

Aust, F., \& Barth, M. (2015). Papaja: Create APA manuscripts with Rmarkdown. Retrieved from Auster, C. J., \& Ohm, S. C. (2000). Masculinity and femininity in contemporary American society: A reevaluation using the bem sex-role inventory.

Sex Roles, 43, 499-528.

\footnotetext{
${ }^{1}$ Note: This document was created using $\boldsymbol{R}$-v3.3.1 (R Core Team, 2016), and the following $\boldsymbol{R}$-packages: base-v3.3. (R Core Team, 2016), bibtex-v0.4. (Francois, 2014), broom-v0.4. (Robinson, 2015), cairoDevice-v2.23. (Lawrence, 2015), car-v2.1. (Fox \& Weisberg, 2011), DBI-v0.5. (Databases, 2014), dplyr-v0.5. (Wickham \& Francois, 2015), DT-v0.2. (Xie, 2015a), extrafont-v0.17. (Chang, 2014), ggplot2v2.1. (Wickham, 2009), gWidgets-v0.0. (Verzani, 2014), gWidgetsRGtk2-v0.0. (Lawrence \& Verzani, 2014), igraph-v1.0. (Csardi \& Nepusz, 2006), knitcitations-v1.0. (Boettiger, 2015), knitr-v1.14. (Xie, 2015b), pander-v0.6. (Daroczi \& Tsegelskyi, 2015), papaja-v0.1. (Aust \& Barth, 2015), plyr-v1.8. (Wickham, 2011), psych-v1.6. (Revelle, 2015), RGtk2-v2.20. (Lawrence \& Temple Lang, 2010), rmarkdown-v1.0. (J. Allaire et al., 2016), RQDA-v0.2. (Huang, 2014), RSQLite-v1.0. (Wickham, James, \& Falcon, 2014), scales-v0.4. (Wickham, 2016b), tidyr-v0.6. (Wickham, 2016c), ggthemes-v3.2. (Arnold, 2016), gtable-v0.2. (Wickham, 2016a), kableExtra-v0.0. (Zhu, 2016), tufte-v0.2. (Xie \& Allaire, 2016), gdata-v2.17. (Warnes et al., 2015), descr-v1.1. (Aquino, 2016), Formula-v1.2. (Zeileis \& Croissant, 2010), Hmisc-v3.17. (Harrell Jr, Charles Dupont, \& others., 2016), irr-v0.84. (Gamer, Lemon, \& Singh, 2012), lattice-v0.20. (Sarkar, 2008), lpSolve-v5.6. (Berkelaar \& others, 2015), vcd-v1.4. (D. Meyer, Zeileis, \& Hornik, 2006), devtools-v1.12. (Wickham \& Chang, 2016), highlight-v0.4. (Francois, 2015), sysfonts-v0.5. (Qiu \& others, 2015), and showtext-v0.4. (Qiu, 2015).
} 
Badenes-Ribera, L., Frias-Navarro, D., Bonilla-Campos, A., Pons-Salvador, G., \& Monterde-i-Bort, H. (2015). Intimate partner violence in self-identified lesbians: A meta-analysis of its prevalence. Sexuality Research and Social Policy, 12, 4759.

Balsam, K. F. (2001). Nowhere to hide: Lesbian battering, homophobia, and minority stress. Women and Therapy, 23, 25-37.

Balsam, K. F., \& Szymanski, D. (2005). Relationship quality and domestic violence in women's same-sex relationships: The role of minority stress. Psychology of Women Quarterly, 29, 258-269.

Banerjee, M., Capozzoli, M., McSweeney, L., \& Sinha, D. (1999). Beyond kappa: A review of interrater agreement measures. Canadian Journal of Statistics, 27, 323.

Bargad, A., \& Hyde, J. S. (1991). Women's studies: A study of feminist identity development in women. Psychology of Women Quarterly, 15, 181-201.

Barry, H. I., Bacon, M., \& Child, I. L. (1957). A cross-cultural survey of some sex differences in socialization. The Journal of Abnormal and Social Psychology, 55, 327.

Bem, S. L. (1977). On the utility of alternative procedures for assessing psychological androgyny. Journal of Consulting and Clinical Psychology, 45, 196.

Bem, S. L. (1981a). Bem Sex-Role Inventory: Professional manual. Consulting Psychologists Press Palo Alto, CA.

Bem, S. L. (1981b). Gender schema theory: A cognitive account of sex typing. Psychological Review, 88, 354. 
Bem, S. L., \& Lewis, S. A. (1975). Sex role adaptability: One consequence of psychological androgyny. Journal of Personality and Social Psychology, 31, 634.

Berkelaar, M., \& others. (2015). LpSolve: Interface to solve linear integer programs. Retrieved from Black, M. C., Basile, K. C., Breiding, M. J., Smith, S. G., Walters, M. L., Merrick, M. T., \& Stevens, M. (2011). National Intimate Partner And Sexual Violence Survey. Atlanta, GA: Centers for Disease Control and Prevention, National Center for Injury Prevention and Control.

Black, M. C., Basile, K. C., Breiding, M. J., Smith, S. G., Walters, M. L., Merrick, M. T., \& Stevens, M. (2014). The National Intimate Partner and Sexual Violence Survey (NISVS): Detailed state tables, Table 7.4. Atlanta, GA: Centers for Disease Control and Prevention, National Center for Injury Prevention and Control. Retrieved from www.cdc.gov/violenceprevention/nisvs/state tables 74.html Blanchard, R., \& Freund, K. (1983). Measuring masculine gender identity in females. Journal of Consulting and Clinical Psychology, 51, 205.

Blount, J. M. (2006). Fit to teach: Same-sex desire, gender, and school work in the twentieth century. Albany, NY, US: SUNY Press.

Boettiger, C. (2015). knitcitations: Citations for knitr markdown files. Retrieved from Braun, V., \& Clarke, V. (2006). Using thematic analysis in psychology. Qualitative Research in Psychology, 3, 77-101.

Breiding, M. J., Black, M. C., \& Ryan, G. W. (2008). Prevalence and risk factors of intimate partner violence in eighteen US states/territories, 2005. American Journal of Preventive Medicine, 34, 112-118. 
Brooks, V. R. (1981). Minority stress and lesbian women. New York, NY, US: Free Press.

Brown, R. D., Clarke, B., Gortmaker, V., \& Robinson-Keilig, R. (2004). Assessing the campus climate for gay, lesbian, bisexual, and transgender (GLBT) students using a multiple perspectives approach. Journal of College Student Development, 45, 8 26.

Burke, L., \& Follingstad, D. R. (1999). Violence in lesbian and gay relationships: Theory, prevalence, and correlational factors. Clinical Psychology Review, 19, $487-512$.

Bush, D. M. (1992). Women's movements and state policy reform aimed at domestic violence against women: A comparison of the consequences of movement mobilization in the US and india. Gender and Society, 6, 587-608.

Campbell, J. C. (1993). The Danger Assessment Instrument: Risk factors of homicide of and by battered women. Questions and Answers in Lethal and Non-Lethal Violence, 1, 27-38.

Carrigan, T., Connell, B., \& Lee, J. (1985). Toward a new sociology of masculinity. Theory and Society, 14, 551-604.

Cecere, D. J. (1986). The second closet: Battered lesbians. Naming the Violence: Speaking Out About Lesbian Battering, 21-31.

Chang, W. (2014). Extrafont: Tools for using fonts. Retrieved from Charmaz, K. (2006). Constructing Grounded Theory: A Practical Guide Through Qualitative Analysis (Introducing Qualitative Methods Series). Thousand Oaks, CA: SAGE Publications, Inc. 
Charmaz, K. (2011). Grounded theory methods in social justice research. In N. K. Denzin \& Y. S. Lincoln (Eds.), The SAGE handbook of qualitative research (pp. 359380). Thousand Oaks, CA: SAGE Publications, Inc.

Charmaz, K. (2014). Constructing grounded theory. Thousand Oaks, CA: SAGE Publications, Inc.

Chen, H. (2016). VennDiagram: Generate high-resolution venn and euler plots. Retrieved from Cohen, J. (1968). Weighted kappa: Nominal scale agreement provision for scaled disagreement or partial credit. Psychological Bulletin, 70, 213.

Collins, D. (2003). Pretesting survey instruments: An overview of cognitive methods. Quality of Life Research, 12, 229-238.

Connell, R. (2002). On hegemonic masculinity and violence response to jefferson and hall. Theoretical Criminology, 6, 89-99.

Connell, R., \& Connell, R. (2005). Masculinities. Oakland, CA, US: University of California Press.

Connell, R., \& Messerschmidt, J. (2005). Hegemonic masculinity: Rethinking the concept. Gender and Society, 19, 829-859.

Corbin, J., \& Strauss, A. (1990). Basics of qualitative research: Grounded theory procedures and techniques. Basics of Qualitative Research: Grounded Theory Procedures and Techniques, 41.

Crenshaw, K. (1991). Mapping the margins: Intersectionality, identity politics, and violence against women of color. Stanford Law Review, 1241-1299. 
Creswell, J. W. (2012). Qualitative inquiry and research design: Choosing among five approaches. Thousand Oaks, CA: SAGE Publications, Inc.

Csardi, G., \& Nepusz, T. (2006). The igraph software package for complex network research. InterJournal, Complex Systems, 1695. Retrieved from

Daroczi, G., \& Tsegelskyi, R. (2015). Pander: An R pandoc writer. Retrieved from

Databases, R. S. I. G. on. (2014). DBI: R database interface. Retrieved from Davis, K., \& Glass, N. (2011). Reframing the heteronormative constructions of lesbian partner violence. In J. L. Ristock (Ed.), Intimate partner violence in LGBTQ lives (p. 13). New York, NY: Routledge.

DeNavas-Walt, C. (2010). Income, poverty, and health insurance coverage in the united states (2005). Collingdale, PA, US: DIANE Publishing.

DiPlacido, J. (1998). Minority stress among lesbians, gay men, and bisexuals: A consequence of heterosexism, homophobia, and stigmatization. Thousand Oaks, CA: SAGE Publications, Inc.

Donovan, C., \& Hester, M. (2011). Exploring emotion work in domestically abusive relationships. In J. L. Ristock (Ed.), Intimate partner violence in LGBTQ lives. (pp. 81-101). New York, NY: Routledge.

Duffy, K. (2011). There's no pride in domestic violence. In J. L. Ristock (Ed.), Intimate partner violence in LGBTQ lives (p. 258). New York, NY: Routledge.

Durish, P. (2011). Documenting the same sex abuse project, toronto, canada. In J. L. Ristock (Ed.), Intimate partner violence in LGBTQ lives (p. 232). New York, NY: Routledge. 
Eaton, L., Kaufman, M., Fuhrel, A., Cain, D., Cherry, C., Pope, H., \& Kalichman, S. C. (2008). Examining factors co-existing with interpersonal violence in lesbian relationships. Journal of Family Violence, 23, 697-705.

Eisler, R., \& Skidmore, R. (1987). Masculine gender role stress: Scale development and component factors in the appraisal of stressful situations. Behavior Modification, $11,123-36$.

Eisler, R., Franchina, J., Moore, T., Honeycutt, H., \& Rhatigan, D. L. (2000). Masculine gender role stress and intimate abuse: Effects of gender relevance of conflict situations on men's attributions and affective responses. Psychology of Men and Masculinity, 1, 30-36.

Elliot, P. (1996). Shattering illusions: Same-sex domestic violence. Journal of Gay and Lesbian Social Services, 4, 1-8.

Ellis, S. J., Kitzinger, C., \& Wilkinson, S. (2003). Attitudes towards lesbians and gay men and support for lesbian and gay human rights among psychology students. Journal of Homosexuality, 44, 121-138.

Fereday, J., \& Muir-Cochrane, E. (2006). Demonstrating rigor using thematic analysis: A hybrid approach of inductive and deductive coding and theme development. International Journal of Qualitative Methods, 5, 80-92.

Fox, J., \& Weisberg, S. (2011). An R companion to applied regression (2nd ed.). Thousand Oaks CA: Sage. Retrieved from

Francois, R. (2014). Bibtex: Bibtex parser. Retrieved from Friedan, B. (1998). It changed my life: Writings on the women's movement. Cambridge, MA, US: Harvard University Press. 
Friedan, B. (2013). The feminine mystique. New York, NY, US: WW Norton; Company.

Gamer, M., Lemon, J., \& Singh, I. F. P. (2012). irr: Various coefficients of interrater reliability and agreement. Retrieved from Gilbert, P. R. (2002). Discourses of female violence and societal gender stereotypes. Violence Against Women, 8, 1271-1300.

Gillespie, B., \& Eisler, R. (1992). Development of the feminine gender role stress scale: A cognitive-behavioral measure of stress, appraisal, and coping for women. Behavior Modification, 16, 426-438.

Girshick, L. B. (2002). No sugar, no spice: Reflections on research on woman-to-woman sexual violence. Violence Against Women, 8, 1500-1520.

Glass, N., Perrin, N., Hanson, G., Bloom, T., Gardner, E., \& Campbell, J. C. (2008). Risk for reassault in abusive female same-sex relationships. American Journal of Public Health, 98, 1021-1027.

Hammond, N. (1989). Lesbian victims of relationship violence. Women and Therapy, 8, 89-105.

Hanisch, C. (1970). The personal is political. In S. Firestone \& A. Koedt (Eds.), Notes from the second year: Women's liberation (pp. 76-78).

Harrell Jr, F. E., Charles Dupont, \& others. (2016). Hmisc: Harrell miscellaneous. Retrieved from Hart, B. (1986). Lesbian battering: An examination. Naming the Violence: Speaking Out About Lesbian Battering, 173-189.

Hassouneh, D., \& Glass, N. (2008). The influence of gender role stereotyping on women's experiences of female same-sex intimate partner violence. Violence Against Women, 14, 310-325. 
Herek, G. M. (2004). Beyond "homophobia": Thinking about sexual prejudice and stigma in the twenty-first century. Sexuality Research and Social Policy, 1, 6-24.

Ho, A. K., Sidanius, J., Kteily, N., Sheehy-Skeffington, J., Pratto, F., Henkel, K. E., ... Stewart, A. L. (2015). The nature of social dominance orientation: Theorizing and measuring preferences for intergroup inequality using the new SDO scale. Journal of Personality and Social Psychology, 109, 1003-1028.

Holt, C. L., \& Ellis, J. B. (1998). Assessing the current validity of the Bem Sex-Role Inventory. Sex Roles, 39, 929-941.

Huang, R. (2014). RQDA: R-based qualitative data analysis. Retrieved from http://rqda.r-forge.r-project.org/

Hudson, W., \& Ricketts, W. A. (1980). A strategy for the measurement of homophobia. Journal of Homosexuality, 5, 357-372.

Johnson, M. P. (1995). Patriarchal terrorism and common couple violence: Two forms of violence against women. Journal of Marriage and the Family, 283-294.

Johnson, M. P. (2006). Conflict and control gender symmetry and asymmetry in domestic violence. Violence Against Women, 12, 1003-1018.

Keeley, T., Al-Janabi, H., Lorgelly, P., \& Coast, J. (2013). A qualitative assessment of the content validity of the ICECAP-a and EQ-5D-5L and their appropriateness for use in health research. PloS One, 8, e85287.

Kimball, M. M. (2001). Gender similarities and differences as feminist contradictions. Handbook of the Psychology of Women and Gender, 66-83. 
Kimmel, M. S. (2004). Masculinity as homophobia: Fear, shame, and silence in the construction of gender identity. Race, Class, and Gender in the United States: An Integrated Study. Ed. Paula Rothenberg. New York: Worth, 81-93.

Lai-Poon, K. (2011). Beyond good and evil: The social construction of violence in intimate gay relationships. In J. L. Ristock (Ed.), Intimate partner violence in LGBTQ lives (pp. 102-131). New York, NY: Routledge.

Laird, J. (1999). Lesbians and lesbian families: Reflections on theory and practice. New York, NY, US: Columbia University Press.

Landis, J. R., \& Koch, G. G. (1977). The measurement of observer agreement for categorical data. Biometrics, 159-174.

Lawrence, M. (2015). CairoDevice: Embeddable cairo graphics device driver. Retrieved from

Lawrence, M., \& Temple Lang, D. (2010). RGtk2: A graphical user interface toolkit for R. Journal of Statistical Software, 37, 1-52. Retrieved from

Lawrence, M., \& Verzani, J. (2014). GWidgetsRGtk2: Toolkit implementation of gWidgets for rgtk2. Retrieved from LeGates, M. (2001). In their time: A history of feminism in western society. New York, NY: Routledge.

Lehavot, K., \& Simoni, J. M. (2011). The impact of minority stress on mental health and substance use among sexual minority women. Journal of Consulting and Clinical Psychology, 79, 159.

Lehavot, K., King, K. M., \& Simoni, J. M. (2011). Development and validation of a gender expression measure among sexual minority women. Psychology of Women Quarterly, 35, 381-400. 
Lenney, E. (1991). Sex roles: The measurement of masculinity, femininity, and androgyny. In J. Robinson, P. Shaver, \& L. Wrightsman (Eds.), Measures of personality and social psychological attitudes. measures of social psychological attitudes (Vol. 1, pp. 573-660). San Diego, CA, US: Academic Press.

Levant, R. F., \& Richmond, K. (2007). A review of research on masculinity ideologies using the Male Role Norms Inventory. The Journal of Men's Studies, 15, 130146.

Levant, R. F., Hall, R. J., \& Rankin, T. J. (2013). Male Role Norms Inventory-Short Form (MRNI-SF): Development, confirmatory factor analytic investigation of structure, and measurement invariance across gender. Journal Of Counseling Psychology, 60, 228.

Levant, R. F., Rankin, T., Williams, C., Hasan, N., \& Smalley, B. (2010). Evaluation of the factor structure and construct validity of scores on the Male Role Norms Inventory-Revised (MRNI-r). Psychology of Men and Masculinity, 11, 25-37.

Levant, R. F., Richmond, Cook, House, T., \& Aupont, M. (2007). The femininity ideology scale: Factor structure, reliability, convergent and discriminant validity, and social contextual variation. Sex Roles, 57, 373-383. https://doi.org/10.1007/s11199-007-9258-5

Lewins, A., \& Silver, C. (2009). Choosing a CAQDAS package: A working paper, 6th edition. CAQDAS Networking Project and Qualitative Innovations in CAQDAS Project. (QUIC).

Link, B., \& Phelan, J. C. (2001). Conceptualizing stigma. Annual Review of Sociology, $363-385$. 
Little, B., \& Terrance, C. (2010). Perceptions of domestic violence in lesbian relationships: Stereotypes and gender role expectations. Journal of Homosexuality, 57, 429-440.

Lobel, K. (1986). Naming the violence: Speaking out about lesbian battering. Seattle, WA, US: Seal Press.

Lockhart, L., White, B., Causby, V., \& Isaac, A. (1994). Letting out the secret: Violence in lesbian relationships. Journal of Interpersonal Violence, 9, 469-492.

Luddy, G., \& Thompson, H. (1997). Masculinities and violence: A father-son comparison of gender traditionality and perceptions of heterosexual rape. Journal of Family Psychology, 11, 462-477. https://doi.org/10.1037/0893-3200.11.4.462

Malamuth, M., Linz, Heavey, L., Barnes, \& Acker, M. (1995). Using the confluence model of sexual aggression to predict men's conflict with women - a 10-year follow-up-study. Journal of Personality and Social Psychology, 69, 353-369.

Mallory, C., Hasenbush, A., \& Sears, B. (2015). Discrimination and harassment by law enforcement officers in the LGBT community. The Williams Institute.

Mankowski, E., \& Maton, K. (2010). A community psychology of men and masculinity: Historical and conceptual review. American Journal of Community Psychology, $45,73-86$.

Mays, V., \& Cochran, S. D. (2001). Mental health correlates of perceived discrimination among lesbian, gay, and bisexual adults in the united states. American Journal of Public Health, 91, 1869-1876.

McClennen, J. C. (2005). Domestic violence between same-gender partners recent findings and future research. Journal of Interpersonal Violence, 20, 149-154. 
McClennen, J. C., Summers, A. B., \& Daley, J. G. (2002). The lesbian partner abuse scale. Research on Social Work Practice, 12, 277-292.

McHugh, M. L. (2012). Interrater reliability: The kappa statistic. Biochemia Medica, 22, 276-282.

McLaughlin, E., \& Rozee, P. D. (2001). Knowledge about heterosexual versus lesbian battering among lesbians. Women and Therapy, 23, 39-58.

Merrill, G. S. (1996). Ruling the exceptions: Same-sex battering and domestic violence theory. Journal of Gay and Lesbian Social Services, 4, 9-22.

Messick, S. (1995). Validity of psychological assessment: Validation of inferences from persons' responses and performances as scientific inquiry into score meaning. American Psychologist, 50, 741.

Messinger, A. M. (2011). Invisible victims: Same-sex IPV in the National Violence Against Women Survey. Journal Of Interpersonal Violence, 26, 2228-2243.

Meyer, D., Zeileis, A., \& Hornik, K. (2006). Residual-based shadings for visualizing (conditional) independence". Journal of Statistical Software, 17, 1-48. Retrieved from

Meyer, I. H. (1995). Minority stress and mental health in gay men. Journal of Health and Social Behavior, 38-56.

Meyer, I. H. (2003). Prejudice, social stress, and mental health in lesbian, gay, and bisexual populations: Conceptual issues and research evidence. Psychological Bulletin, 129, 674. 
Meyer, I. H. (2010). The right comparisons in testing the minority stress hypothesis: Comment on savin-williams, cohen, joyner, and rieger (2010). Archives of Sexual Behavior, 39, 1217-1219.

Meyer, I. H. (2015). Resilience in the study of minority stress and health of sexual and gender minorities. Psychology of Sexual Orientation and Gender Diversity, 2, 209.

Millett, K. (2000). Sexual Politics. Champaign, IL, US: University of Illinois Press.

Mogul, J. L., Ritchie, A. J., \& Whitlock, K. (2011). Queer (in) justice: The criminalization of LGBT people in the united states (Vol. 5). Boston, MA: Beacon Press.

Mohr, J., \& Fassinger, R. (2000). Measuring dimensions of lesbian and gay male experience. Measurement and Evaluation in Counseling and Development.

Moore, T., \& Stuart, G. L. (2004). Effects of masculine gender role stress on men's cognitive, affective, physiological, and aggressive responses to intimate conflict situations. Psychology of Men and Masculinity, 5, 132.

Moore, T., \& Stuart, G. L. (2005). A review of the literature on masculinity and partner violence. Psychology of Men and Masculinity, 6, 46.

Moore, T., Stuart, G., McNulty, J., Addis, M., Cordova, J., \& Temple, J. R. (2010). Domains of masculine gender role stress and intimate partner violence in a clinical sample of violent men. Psychology of Violence, 1, 68-75. https://doi.org/10.1037/2152-0828.1.S.68

Morgan, D. (1996a). Focus groups. Annual Review of Sociology, 129-152. 
Morgan, D. (1996b). Focus groups as qualitative research (Vol. 16). Thousand Oaks, CA: Sage publications.

Morgan, D. (2014). Integrating qualitative and quantitative methods: A pragmatic approach (Vol. 16). Thousand Oaks, CA: SAGE Publications, Inc.

Morrow, S., \& Hawxhurst, D. M. (1989). Lesbian partner abuse: Implications for therapists. Journal of Counseling and Development, 68, 58-62.

Multon, K. (2010). Interrater reliability. In N. J. Salkind (Ed.), Encyclopedia of research design (Vol. 2, pp. 626-628). Thousand Oaks, CA: SAGE Publications, Inc.

Murnen, S., Wright, \& Kaluzny, G. (2002). If "boys will be boys," then girls will be victims? A meta-analytic review of the research that relates masculine ideology to sexual aggression. Sex Roles, 46, 359-375.

Myers, A. M., \& Gonda, G. (1982). Empirical validation of the bem sex-role inventory. Journal of Personality and Social Psychology, 43, 304.

Newton, E. (1984). The mythic mannish lesbian: Radclyffe hall and the new woman. Signs, 557-575.

Onwuegbuzie, A. J., Dickinson, W. B., Leech, N. L., \& Zoran, A. G. (2009). A qualitative framework for collecting and analyzing data in focus group research. International Journal of Qualitative Methods, 8, 1-21.

O’Neil, J. M. (1981). Patterns of gender role conflict and strain: Sexism and fear of femininity in men's lives. The Personnel and Guidance Journal, 60, 203-210.

O’Neil, J. M. (2008). Summarizing 25 years of research on men's gender role conflict using the gender role conflict scale new research paradigms and clinical implications. The Counseling Psychologist, 36, 358-445. 
Pattavina, A., Hirschel, D., Buzawa, E., Faggiani, D., \& Bentley, H. (2007). A comparison of the police response to heterosexual versus same-sex intimate partner violence. Violence Against Women, 13, 374-394.

Pence, E. (1983). Duluth domestic abuse intervention project, the. Hamline Rev., 6, 247.

Pinel, E. C. (1999). Stigma consciousness: The psychological legacy of social stereotypes. Journal of Personality and Social Psychology, 76, 114.

Pleck, E. H. (1987). Domestic tyranny: The making of social policy against family violence from colonial times to the present. Oxford University Press New York.

R Core Team. (2016). R: A language and environment for statistical computing. Vienna, Austria: R Foundation for Statistical Computing. Retrieved from https://www.Rproject.org/

Renzetti, C. M. (1992). Violent betrayal: Partner abuse in lesbian relationships. Thousand Oaks, CA: SAGE Publications, Inc.

Renzetti, C. M. (1998). Violence and abuse in lesbian relationships. In R. K. Bergen (Ed.), Issues in intimate violence (pp. 117-127). Thousand Oaks, CA: Sage Publications Inc.

Revelle, W. (2015). Psych: Procedures for psychological, psychometric, and personality research. Evanston, Illinois: Northwestern University. Retrieved from Ristock, J. L. (2001). Decentering heterosexuality: Responses of feminist counselors to abuse in lesbian relationships. Women and Therapy, 23, 59-72.

Ristock, J. L. (2002). No more secrets: Violence in lesbian relationships. Psychology Press. 
Ristock, J. L. (2003). Exploring dynamics of abusive lesbian relationships: Preliminary analysis of a multisite, qualitative study. American Journal of Community Psychology, 31, 329-341.

Ristock, J. L. (2011). Intimate partner violence in LGBTQ lives. New York, NY: Routledge.

Robinson, D. (2015). Broom: Convert statistical analysis objects into tidy data frames. Retrieved from Robson, R. (1990). Lavender bruises: Intra-lesbian violence, law and lesbian legal theory. Golden Gate UL Rev., 20, 567.

Rowe, B. L. Y. (2015). Futile.logger: A logging utility for $r$. Retrieved from Saldaña, J. (2015). The coding manual for qualitative researchers. Thousand Oaks, CA: SAGE Publications, Inc.

Sarkar, D. (2008). Lattice: Multivariate data visualization with R. New York: Springer. Retrieved from Sheffield, C. J. (1987). Sexual terrorism: The social control of women. Thousand Oaks, CA: Sage Publications, Inc.

Shidlo, A. (1994). Internalized homophobia: Conceptual and empirical issues in measurement. In Greene \& M. Herek (Eds.), Lesbian and gay psychology: Theory, research, and clinical applications (pp. 176-205). Thousand Oaks, CA: Sage Publications, Inc.

Sidanius, J., \& Pratto, F. (2001). Social dominance: An intergroup theory of social hierarchy and oppression. New York, NY, US: Cambridge University Press.

Silverstein, C. (2009). The implications of removing homosexuality from the DSM as a mental disorder. Archives of Sexual Behavior, 38, 161-163. 
Sim, J., \& Wright, C. C. (2005). The kappa statistic in reliability studies: Use, interpretation, and sample size requirements. Physical Therapy, 85, 257-268.

Smith, C. (2011). Women who abuse their female intimate partners. In J. L. Ristock (Ed.), Intimate partner violence in LGBTQ lives (pp. 131-52). New York, NY: Routledge.

Smith, R. M., Parrott, D. J., Swartout, K. M., \& Tharp, A. T. (2015). Deconstructing hegemonic masculinity: The roles of antifemininity, subordination to women, and sexual dominance in men's perpetration of sexual aggression. Psychology of Men and Masculinity, 16, 160-169.

Sokoloff, N., \& Pratt, C. (2005). Domestic violence at the margins: Readings on race, class, gender, and culture. Rutgers University Press.

Spaulding, E. (1999). Unconsciousness-raising: Hidden dimensions of heterosexism in theory and practice with lesbians. Lesbians and Lesbian Families: Reflections on Theory and Practice, 11-26.

Spence, J., \& Helmreich, R. L. (1980). Masculine instrumentality and feminine expressiveness: Their relationships with sex role attitudes and behaviors. Psychology of Women Quarterly, 5, 147-163.

Straus, M. A. (1979). Measuring intrafamily conflict and violence: The conflict tactics (CT) scales. Journal of Marriage and the Family, 75-88.

Straus, M. A., Hamby, S. L., BoneyMcCoy, S., \& Sugarman, D. B. (1996). The revised Conflict Tactics Scales (CTS2): Development and preliminary psychometric data. Journal of Family Issues, 17, 283-316. 
Strauss, A., \& Corbin, J. (1990). Basics of qualitative research (Vol. 15). Thousand Oaks, CA: SAGE Publications, Inc.

Strauss, A., \& Corbin, J. (1994). Grounded theory methodology. Handbook of Qualitative Research, 273-285.

Sue, D. W. (2010). Microaggressions and marginality: Manifestation, dynamics, and impact. Hoboken, New Jersey: John Wiley; Sons.

Sue, D. W., Capodilupo, C. M., Torino, G. C., Bucceri, J. M., Holder, A., Nadal, K. L., \& Esquilin, M. (2007). Racial microaggressions in everyday life: Implications for clinical practice. American Psychologist, 62, 271.

Szymanski, D., \& Chung, B. (2008). The lesbian internalized homophobia scale: A rational/theoretical approach. Journal of Homosexuality, 41, 37-52.

Szymanski, D., Chung, B., \& Balsam, K. F. (2001). Psychosocial correlates of internalized homophobia in lesbians. Measurement and Evaluation in Counseling and Development.

Terman, L. M., \& Miles, C. C. (1936). Sex and personality: Studies in masculinity and femininity. New York, NY: McGraw-Hill.

Tjaden, P., \& Thoennes, N. (2000a). Full report of the prevalence, incidence, and consequences of violence against women: Findings from the National Violence against Women Survey: Research report. Washington, DC: National Institute of Justice.

Tjaden, P., \& Thoennes, N. (2000b). Prevalence and consequences of male-to-female and female-to-male intimate partner violence as measured by the National Violence against Women Survey. Violence Against Women, 6, 142-161. 
Tjaden, P., Thoennes, N., \& Allison, C. J. (1999). Comparing violence over the life span in samples of same-sex and opposite-sex cohabitants. Violence and Victims, 14, 413-425.

Truman, D., Tokar, D., \& Fischer, A. R. (1996). Dimensions of masculinity: Relations to date rape supportive attitudes and sexual aggression in dating situations. Journal of Counseling and Development, 74, 555-562.

Urbanek, S. (2016). RJava: Low level R to java interface. Retrieved from Vance, B., \& Green, V. (1984). Lesbian identities: An examination of sexual behavior and sex. role attribution as related to age of initial same-sex sexual encounter. Psychology of Women Quarterly, 8, 293-307.

VanNatta, M. (2005). Constructing the battered woman. Feminist Studies, 416-443.

Verzani, J. (2014). GWidgets: GWidgets api for building toolkit-independent, interactive guis. Retrieved from Viera, A., Garrett, J., \& others. (2005). Understanding interobserver agreement: The kappa statistic. Fam Med, 37, 360-363.

Vogt, D., King, D., \& King, L. A. (2004). Focus groups in psychological assessment: Enhancing content validity by consulting members of the target population. Psychological Assessment, 16, 231.

Wagner, G. J. (1998). Internalized homophobia scale. Handbook of Sexuality-Related Measures, 371-372.

Walters, M. L., Chen, J., \& Breiding, M. J. (2013). The national intimate partner and sexual violence survey (NISVS): 2010 findings on victimization by sexual orientation. Atlanta, GA: Centers for Disease Control and Prevention. 
Warnes, G. R., Bolker, B., Gorjanc, G., Grothendieck, G., Korosec, A., Lumley, T., ... others. (2015). Gdata: Various r programming tools for data manipulation. Retrieved from Wetherell, M., \& Edley, N. (1999). Negotiating hegemonic masculinity: Imaginary positions and psycho-discursive practices. Feminism and Psychology, 9, 335-356.

Wickham, H. (2009). ggplot2: Elegant graphics for data analysis. Springer-Verlag New York. Retrieved from

Wickham, H. (2011). The split-apply-combine strategy for data analysis. Journal of Statistical Software, 40, 1-29. Retrieved from

Wickham, H. (2016a). Gtable: Arrange grobs in tables. Retrieved from

Wickham, H. (2016b). Scales: Scale functions for visualization. Retrieved from

Wickham, H. (2016c). Tidyr: Easily tidy data with spread() and gather() functions. Retrieved from

Wickham, H., \& Chang, W. (2016). Devtools: Tools to make developing r packages easier. Retrieved from

Wickham, H., \& Francois, R. (2015). Dplyr: A grammar of data manipulation. Retrieved from

Wickham, H., James, D. A., \& Falcon, S. (2014). RSQLite: SQLite interface for $r$. Retrieved from

Wilkinson, L. (2011). Venneuler: Venn and euler diagrams. Retrieved from Xie, Y. (2015a). DT: A wrapper of the javascript library datatables. Retrieved from Xie, Y. (2015b). Dynamic documents with $R$ and knitr (2nd ed.). Boca Raton, Florida: Chapman; Hall/CRC. Retrieved from 
Xie, Y., \& Allaire, J. (2016). Tufte: Tufte's styles for $r$ markdown documents. Retrieved from

Zeileis, A., \& Croissant, Y. (2010). Extended model formulas in R: Multiple parts and multiple responses. Journal of Statistical Software, 34(1), 1-13. Retrieved from

Zhu, H. (2016). KableExtra: Decorate kable output using pipe syntax. Retrieved from Zurbriggen, E. L. (2010). Rape, war, and the socialization of masculinity: Why our refusal to give up war ensures that rape cannot be eradicated. Psychology of Women Quarterly, 34, 538-549. 
Appendices 


\section{Appendix A. Focus Group Guide}

\section{Appendix A1. Focus Groups Interview Introduction}

\section{Welcome and Introduction}

"Thank you all for joining us today! To give you a little more information about the study, we are inviting individuals to participate in focus groups for a research project aimed at understanding sexual minority women's experiences with female same-sex intimate partner violence. Some of these focus groups will be with Lesbian, Bisexual, and Queer women recruited from the greater Portland area, while others will be with sexual minority women who are currently receiving community services related to intimate partner violence. All of the focus groups will consist of discussion questions about stereotypes participants have heard related to sexual minority women and their thoughts on these stereotypes. While this study is ultimately interested in addressing intimate partner violence in samesex couples, no specific questions will be asked about violence. Each focus group meeting is expected to last for approximately 2 hours."

\section{Informed Consent}

"Before we get started, we need to go over the informed consent document that is on the table in front of each of you. We would like for each of you to read through the document on your own after I highlight the main points for the group. First, the goal of today's focus group is to learn people's thoughts about stereotypes attributed to Lesbian, Bisexual, and Queer women and how those stereotypes, and the experience of being stereotyped, relate or intersect with their day-to-day lives. You will receive a \$30 gift card to Powell's Books in appreciation of your time and participation. If you decide to leave for any reason before the group is finished you will still receive a \$30 gift card to Powell's Books. Your participation in this research is meant to be an opportunity for you to share your individual thoughts around these topics and to hear those of others who may have been, in some way or another, silenced. You may not receive any direct benefit from this research, but the knowledge it generates may help others in the future. This focus group interview will be recorded using a digital voice recorder and a written record, or transcript, will be made from the recording. The information in the transcript from today's focus group will not be connected to your name and the best efforts will be made to protect your privacy and confidentiality. However, confidentiality cannot be guaranteed in a group setting and it is possible some of the things you say in today's focus group could be repeated by others in today's group. So, two of the best ways to ensure your privacy are (1) refrain from saying things you would not want others to know and (2) refrain from repeating anything said by other group members."

"Does anyone have any initial questions?" 
"If everyone could take a minute to read through this consent form on your own and, if you agree to participate in today's focus group, sign the consent form and return it to me. A copy of this document has also been provided on the table in front of each of you and this copy is for you to keep."

"Please feel free to ask any questions before signing this form."

\section{Ground Rules}

"Thank you all for taking the time to be here today. Before we begin, I would like to just go over some basic ground rules for today's group. These are things about talking in groups that are just general requests we are making of everyone present today to make sure that everyone feels comfortable and safe in today's discussion."

"The first thing is to participate. Again, the reason that we've invited all of you here today is so we can hear your different points of view. So we need everybody's help to have a good group."

"The second thing is to take turns. We know that some people like to talk more than others, but you may have to hold on to some of the things you'd like to say, so that everyone in the group has a chance to talk."

"Third, it's all right to disagree with each other, but please be polite when you do - no put downs. Everyone wants other people to listen when you talk and to show some respect for you, right? So you need to do the same for them." "Finally, as I mentioned earlier, confidentiality cannot be guaranteed in a group setting, so we are asking that everyone understands that the best ways to protect your own privacy and confidentiality are to (1) not say anything about yourself that you would not want others to know, and (2) to not repeat anything said by other group members." 


\section{Appendix A2. Focus Groups Moderating Guide}

\section{Q1 - Getting Acquainted \& Introductory activity}

Why don't we start by having everyone introduce themselves with their names, how they found out about today's group [e.g., a flyer at the QRC], and oneto-two words that best describe your gender identity.

Now that we have gotten a little more acquainted with each other, I am interested in hearing about your experiences with stereotypes related to your sexual and gender identities. So first, I'd like for you to think about some of the stereotypes you have heard of related to lesbian, bisexual, and queer women.

TO START, take one of the note cards from the stack on the table and write down as many of these stereotypes you can think of. Try using only one or two words for each of the stereotypes, because we are going to list what you write down on the easel pad. Keep in mind that these are stereotypes you have heard of and we are not assuming anything about whether or not you endorse these stereotypes as truths.

- AfTER 2-3 MINUTES, tell participants to wrap-up their writing and to start listing, out loud, the stereotypes they came up with, in popcorn style, and record their responses on the easel pad using as many pages as necessary.

\section{Q2 - Cued Discussion Starter}

What do you all think about these stereotypes and their influence on your lives and identities?

\section{Q3 - Cued Discussion Starter}

Now that we have this list, what are your thoughts about some of these stereotypes? Which ones seem to be pretty accurate or inaccurate and why?

- PROMPT for details and specific stories when applicable.

\section{Q4 - Indirect Gender Role Cues}

(if not brought up organically via Questions 1 \& 2): What are some ways you feel your gender identity and gender expression(s) have influenced your dayto-day life?

\section{Q5 - Indirect Gender Role Cues}

What are some of the ways this influence has been a positive aspect of your life? How about less positive or negative ways this has influenced your life?

\section{Q6 - Semi-direct Gender Role Cues}

How has your sexual orientation influenced your gender identity? 
What are some ways that these influences have been difficult or caused problems in your life?

- PROMPT for terms like 'stress' and 'strain' if they have not come up yet.

\section{Q7 - Wrap-up}

Why don't we try to think of ways that those difficulties could be eliminated: could each of you think of, and share, one or two things that could reduce those difficulties? 


\section{Appendix B. One-on-One Interview Guide}

\section{Appendix B1. One-on-One Interview Introduction}

\section{Welcome and Introduction}

THANK YOU FOR JOINING ME TODAY! To give you a little more information about the study, we are inviting individuals to participate in focus groups and one-onone interviews for a research project aimed at understanding LGBTQ Women's experiences with female same-sex intimate partner violence.

Some of these interviews will be with LGBTQ women recruited from the greater Portland area, while others will be with sexual minority women who are currently receiving community services related to intimate partner violence.

All of the interviews will consist of questions about stereotypes participants have heard related to sexual minority women and their thoughts on these stereotypes. While this study is ultimately interested in addressing intimate partner violence in same-sex couples, no specific questions will be asked about violence.

Today's meeting is expected to last approximately one hour.

\section{Informed Consent}

Before we get started, we need to go over the informed consent document that has been provided to you.

I would like for you to read through the document on your own after I highlight the main points for you.

First, the goal of today's interview is to learn your thoughts about stereotypes attributed to Lesbian, Bisexual, and Queer women and how those stereotypes, and the experience of being stereotyped, relate or intersect with your day-to-day life. You will receive a $\$ 15$ gift card to Powell's Books in appreciation of your time and participation.

If you decide to leave for any reason before the interview is finished you will still receive a \$15 gift card to Powell's Books. Your participation in this research is meant to be an opportunity for you to share your thoughts around these topics. You may not receive any direct benefit from this research, but the knowledge it generates may help others in the future.

This interview will be recorded using a digital voice recorder and a written record, or transcript, will be made from the recording.

The information in the transcript from today's interview will not be connected to your name and the best efforts will be made to protect your privacy and confidentiality.

Do you have any initial questions?

If you could take a minute to read through this consent form on your own and, if you agree to participate in today's interview, sign the consent form and return it to me. 
The second copy of this document I have provided is for you to keep. Please feel free to ask any questions before signing this form. 


\section{Appendix B2. One-on-One Interview Guide}

\section{Introductory Activity}

I am interested in hearing about your experiences with stereotypes related to your sexual and gender identity. So first, I'd like for you to think about some of the stereotypes you have heard of related to lesbian, bisexual, and queer women.

To start, take one of the note cards from the stack on the table and write down as many of these stereotypes you can think of.

In the interest of time, try using only one or two words for each of the stereotypes. Keep in mind that these are stereotypes you have heard of and I am not assuming anything about whether or not you endorse these stereotypes as truths.

- AFTER 2-3 MINUTES, ask participant to wrap-up her writing and continue with the interview:

\section{Q1 - Stereotypes}

\section{What do you think about these stereotypes?}

Which ones seem to be pretty accurate?

Why?

Which ones seem to be pretty inaccurate?

Why?

How have these stereotypes influenced, or not influenced, your life?

- $\quad$ PRompt for details and specific stories when applicable.

\section{Q2 - Identity}

How have the stereotypes you wrote down influenced, or not influenced, your identity?

What comes to mind when I say your identity?

If you had to choose 5 words that best describe your identity, what would those be?

HELPER CUE, IF NECESSARY: For example, the five words I might choose to describe my own identity are woman, queer, lesbian, white, and researcher.

- ONCE RESPONDENT LISTS HER 5 WORDS, ASK:

Are there any words that you did not include that also describe your identity?

- $\quad$ [IF YES] What are they?

How did you choose which words to include in your list of 5 and which words not to include?

What criteria, if any, did you use to determine which words to keep and which to exclude?

Did you feel that deciding which words to include was difficult?

- $\quad$ [IF YES] How was it difficult?

- $\quad[I F N O]$ How was it not difficult? 
How would you describe your gender identity?

What do you think of when I say gender identity?

How would you describe your gender expression?

What do you think of when I say gender expression?

\section{Q3 - Gender Roles}

What are some ways you feel your gender identity and gender expression(s) have influenced your day-to-day life?

What are some of the ways this influence has been a positive aspect of your life? How about less positive, or negative, ways this has influenced your life?

- $\quad$ PROMPT for terms like role(s), and perform, performance if they do not come up.

\section{Q4 Gender Role Stress \& Minority Stress}

How has your sexual orientation influenced your gender identity and gender expression(s), or the other way around'?

What are some of the ways, if any, this influence has been positive for you? What are some ways, if any, that these influences have been difficult or caused problems in your life?

- PROMPT for terms like stress, anxiety, and strain if they do not come up.

\section{Q5 - Wrap-up}

Could you think of, and share with me, some ways that these difficulties and problems could be reduced or avoided?

IF PARTICIPANT DID NOT NAME ANY DIFFICULTIES OR PROBLEMS, ASK:

In your experience, what do you think has helped lessen or prevent any negative experiences or issues stemming from the influence of your sexual orientation on your gender identity and gender expression(s)? 


\section{Appendix C. Inductively-Derived Codebook}

\section{Appendix C1. Codebook Categories}

\section{MinORity STRESS (MS).}

Relating to or reflecting the loss or diminishing of choice regarding how an individual self-defines and expresses their identity or facets of their identity due to having one or multiple marginalized identities.

\section{GeNDER (G).}

Relating to or reflecting the influence of gender as a form of social categorization or as a personal identity characteristic on an individual's overall identity or specific components of a person's identity, such as their, sexual, racial, ethnic, religious, and/or political identities, etc. (e.g., "I feel most comfortable hanging out with other women", "I feel like I am one of the guys", "I do not really think about my gender or others' genders", etc.). Items reflecting this code can refer to the general concept of gender as a form of social categorization.

\section{IDENTITY (ID).}

An individual's self-defined identity or identity components. This category reflects both the form of an individual's identity definition in terms of the extent to which an individual conceptualizes her identity as either hierarchical or faceted, as well as the context(s) influencing an individual's identity definition(s) and expression(s).

\section{INTERSECTIONALITY}

Relating to or reflecting the notion that individuals identities comprise multiple components or dimensions that are neither mutually exclusive nor necessarily distinct from one another, but that intersect with one another in various ways. The intersectionality category includes four sub-categories each reflecting intersections among the above defined codebook categories: Identity $\boldsymbol{x}$ Gender (IDxG), Minority Stress $\boldsymbol{x}$ Gender (MSxG), Minority Stress $\boldsymbol{x}$ Identity (MSxID), and Minority Stress $\boldsymbol{x}$ Identity $\boldsymbol{x}$ Gender (MSxIDxG).

\section{IDENTITY X GENDER (ID X G).}

Intersections among an individual's identity or identity components and the influence of the individual's gender identity, or of gender as a form of social categorization, on the individual's experiences and behaviors.

\section{MINORITY STRESS X GENDER (MS X G)}

Intersections among minority stress experiences, in the form of the loss or diminishing of choice in one's identity definition(s) and expression(s), and the influence of the individual's gender identity, or of gender as a form of social categorization, on the individual's experiences and behaviors. 
MINORITY STRESS X IDENTITY (MS X ID).

Intersections among minority stress experiences, in the form of the loss or diminishing of choice in one's identity definition(s) and expression(s), and an individual's overall identity or components of an individual's identity.

\section{MINORITY STRESS X IDENTITY X GENDER (MS X ID X G).}

Intersections among minority stress experiences, in the form of the loss or diminishing of choice in one's identity definition(s) and expression(s), an individual's overall identity or components of an individual's identity, and the influence of the individual's gender identity, or of gender as a form of social categorization, on the individual's experiences and behaviors. 


\section{Appendix C2. Codelist}

\section{Gender}

\section{ANDROGYNY.}

Gender expression (GREX) that is a balanced mixture of femininity and masculinity.

\section{BUTCH.}

A form of masculine gender identity or gender expression that has historically been common among sexual minority women.

\section{Cis-Gender.}

A form of gender identity characterized by an individual's current gender identity matching the gender or sex they were assigned at birth.

\section{CONSISTENCY.}

Demonstrating consistency in how one identifies, expresses oneself, or behaves in a given context.

\section{Culture.}

Relating to experiences, identities, or behaviors that explicitly reflect ones' identification with a specific culture.

\section{Dominant Culture.}

Relating to one's experiences with the dominant culture (i.e., white, heteronormative, patriarchy).

\section{Equitable Roles.}

Referring to an equitable distribution of roles within a romantic relationship.

\section{EXPRESSION.}

Relating to an individual's self-expression(s) not specific to one's gender expression. Self-expressions can include expressing (or restricting the expression of) one's emotions, thoughts, etc.

\section{Female.}

Gender identity labeled (by others or the subject) or assigned (at birth) as female.

\section{FLUIDITY.}

Relating to or reflecting fluidity or adaptability in how an individual identifies or expresses herself.

\section{Gender Role STRESS.}

Relating to or reflecting stress, anxiety, conflict, or strain in response to failing or struggling to successfully adhere to a particular set of gender roles. 
GENDERED LANGUAGE.

The use of gender in language as a way of describing or distinguishing an individual or group.

INDEPENDENCE.

Relating to or reflecting an individual's sense of independence, self-sufficiency, or self-reliance (e.g., I can take care of myself).

Media-Online Forums.

Descriptions of online forums as either a source of information or a space of community.

NATURE OVER NURTURE.

Relating to or reflecting the idea that individuals' behaviors and abilities are primarily biologically determined rather than learned through socialization or cultural norms.

\section{Presentation.}

Relating to an individual's overall self-presentation (i.e., the combination of an individual's self-expression, appearance, or role performance)

\section{ROLES.}

Relating to or reflecting the roles an individual is expected to adhere to, does adhere to, struggles to adhere to, or refuses to adhere to. The concept of roles is an overarching category that is inclusive of gendered and non-gendered roles.

\section{TWO-SPIRIT.}

Explicitly relating or referring to the Indegenous American identity two-spirit.

\section{WOMAN.}

Gender identity labeled (by others or the subject) as woman.

\section{Identity}

AGE.

Relating to an individual's age.

\section{AuthentiCITY.}

Being true to oneself. Authenticity can be reflected in many ways, including the way an individual expresses herself, standing up for one's beliefs, etc.

\section{Community.}

Relating to a sense of community or group membership.

\section{EXTERNAL INFLUENCE.}

Relating to or reflecting external influences on an individual's identity, identity definition(s), experiences, or behaviors. 
FEMINISM.

Explicit description of feminism as a political or social change movement, or descriptions of oneself as a feminist (e.g., I identify as a feminist).

\section{INTELLECT.}

Relating or referring to an individual's intelligence. This can include how others perceive the subject's intelligence or the subject's own description of her intelligence. Synonymous terms include adjectives such as "analytical", "aptitude", "smart", etc. Reverse-coding of intelligence can be applied to adjectives such as "gullible", "stupid", "shallow", "inept", etc.

\section{LGBTQ HISTORY.}

Explicitly relating or referring to the LGBTQ-specific historical moments (e.g., the Stonewall Riots), icons (e.g., Harvey Milk), or time periods (e.g., the Stonewall Era).

\section{Media InFLUENCE.}

Descriptions of the level of influence of media on an individual's experiences, behaviors, or identity definitions.

\section{OTHERS' CONFUSION.}

Relating to or reflecting others' confusion about an individual.

\section{Parenting.}

Explicitly relating or referring to parenting or the possibility of parenting.

\section{Priority IDENTITY.}

Explicit description of or reference to a single domain of an individual's identity as the priority identity domain for the individual (e.g., "My race always comes first").

\section{RACE.}

Relating to or reflecting either the general concept of race, or the influence of race or an individual's racial identity on an individual's overall identity, an individual's racial identity, components of an individual's identity other than race, etc. (e.g., "I feel most comfortable in the Black Lesbian Community", "I do not associate with others outside of my own racial group", "I do not really think about my race or others' race", etc.).

\section{SELF-PERCEPTION.}

Relating to or reflecting the way an individual sees or understands herself, regardless of how others may or may not perceive her.

\section{SELF-PRESENTATION.}

Relating to or reflecting the way an individual presents herself regardless of others' expectations or other external influences.

\section{SEXUALITY.}

Relating to or reflecting an individual's sexuality, including an individual's sexual preferences (i.e., attraction) or sexual behaviors. 
Social Media.

Descriptions of social media as either influencing an individual's identity or behaviors, a source of information, or a space of community.

\section{TitLE.}

Explicitly relating or referring to an individual's professional title (e.g., Teacher, Writer, Mechanic, etc.).

\section{Minority Stress}

\section{ACCeptance.}

Seeking or receiving acceptance from others.

\section{AdulthoOD.}

Adulthood-specific experiences or behaviors.

\section{COMPLiance.}

Relating to an individual's compliance or conformity to others' expectations.

\section{DAY-TO-DAY.}

Relating in general to one's day-to-day experiences (i.e., regardless of any specific context or behaviors).

\section{Educating Others.}

Relating to the need, or perceiced need, to educate others about one's marginalized identity (or identities) or experiences.

\section{GENDER Roles.}

Social constructed behaviors, expressions, or beliefs individuals of a given gender identity are expected to demonstrate or adhere to.

\section{Gendered Clothing.}

Descriptions of clothing in direct relation to gender (e.g. wearing skirt makes me feel feminine) or as synonymous with or analogous to a form of gender expression (e.g. wearing a skirt is feminine).

\section{HETERONORMATIVITY.}

Relating to or reflecting the idea that heterosexuality is the only normal sexual orientation or is a default sexual orientation to which others are referred or compared.

\section{HYPERSEXUALIZED.}

Relating to or reflecting the hypersexualization of an individual or group.

\section{IDENTITY WORK.}

Relating to or reflecting an individual's explorations in defining or expressing her identity or facets of her identity. This can include descriptions of how an individual 
came to define or express her identity, experimenting with how she expresses her identity, learning to be comfortable with her identity, etc.

\section{INCLUSION.}

Relating to or reflecting an individual's sense of inclusion in a given context (e.g., "I feel that I am a part of the LGBTQ community").

\section{IPV.}

Relating to or reflecting experiences of Intimate Partner Violence victimization or perpetration.

\section{OTHERS' REACTIONS.}

Relating to or reflecting others' reactions to an individual.

\section{Privilege.}

Relating to or reflecting an individual's privileged identities or experiences of privilege.

\section{SAFETY.}

Relating to or reflecting an individual's sense of safety in a given context.

\section{SCHOOL EXPERIENCES.}

Relating or referring to experiences at school (i.e., primary school, high school, college, graduate school, etc.)

\section{SOCIAL SUPPORT.}

Relating to or reflecting either the need for social support, seeking social support from others, or providing social support to others.

\section{SPACE.}

Relating to or reflecting the concept of "space" as an environment not necessarily confined to a physical place, but rather determined by the individuals occupying a given environment or characteristics that make up an environment (e.g., I do not feel included in LGBTQ spaces).

\section{SuRVIVAL.}

Relating or referring to an individual's ability to survive, strategies used to survive, or barriers to an individual's survival.

\section{Within GROUP OPPRESSION.}

Relating or referring to experiences of oppression from within an individual's social or cultural group.

\section{Within Group Stereotyping.}

Relating or referring to experiences of being stereotyped by or stereotyping others from within an individual's social or cultural group. 


\section{Intersectionality: Identity x Gender}

\section{Change.}

Relating to an individual's experiences of or ability to change.

\section{Confusion.}

Experiencing confusion in one's identity or self-expression.

\section{GENDER Role ENFORCEMENT.}

Relating to or reflecting others' enforcement of an individual's adherence to a particular set of roles (e.g., behaviors, expressions, appearance, etc.) based on the perceived gender of the individual.

\section{HiSTORY.}

Explicitly relating or referring to social, cultural, or political history or histories.This can be reference to a specific historical event or period, or reference to the subject of history in general.

\section{Identity Policing.}

Relating to or reflecting experiences of having one's identity policed or enforced by others. This is separate from role enforcement in that identity policing specifically refers to the definition of one's identity while role enforcement refers to the behaviors, beliefs, or expressions associated with one's identity.

\section{LABELS.}

Relating to or reflecting experiences of being labeled by others.

\section{Personality.}

Descriptions of an individual's personality or personal characteristics not specific to any one facet of an individual's identity such as gender, race, religion, etc. (e.g., "I am friendly").

\section{Intersectionality: Minority Stress x Gender}

\section{Appearance-Clothing.}

Relating to an individual's physical appearance specifically in terms of clothing or apparel.

\section{BINARY.}

Relating to or reflecting a binary understanding of gender (i.e., male/masculine + female/feminine). Items relevant to or reflecting the gender binary are those that 
specifically identify both male (or masculine) and female (or feminine) as the only two possibilities for gender identity, gender expression, and/or gender roles.

\section{FEMININITY.}

Gender expression(s) or gender role(s) explicitly described as feminine or explicitly B55labeled as femininity.

\section{LGBTQ COMMUNITY.}

Explicitly relating or referring to the LGBTQ Community.

\section{Masculinity.}

Gender expression(s) or gender role(s) described as feminine or labeled as femininity.

\section{Misgendering.}

Relating or referring to experiences of being mis-gendered by others (e.g., an individual who identifies as female being called "sir", an individual who identifies as non-binary being labeled as either female or male, etc.).

\section{ROMANTIC LIFE.}

Relating to or reflecting the roles an individual is expected to adhere to, does adhere to, struggles to adhere to, or refuses to adhere to. The concept of roles is an overarching category that is inclusive of gendered and non-gendered roles.

\section{Social Change.}

Relating or referring to social change (e.g., Things are getting better for LGBTQ people).

\section{Intersectionality: Minority Stress x Identity}

\section{CONFIDENCE.}

Seeking or experiencing confidence in oneself.

\section{GENDER EXPRESSION.}

Relating to or reflecting an individual's expression of her gender identity.

\section{LoOking The PART.}

Relating to or reflecting an individual's performance or attempts to perform a particular role or set of roles specifically in terms of how the individual appears or attempts to appear to others.

\section{Mental Health.}

Relating to or reflecting an individual's mental health or mental health concerns.

\section{Pressure.}

Relating to or reflecting experiences of pressure to respond or adhere to others' expectations. 
RACE IDENTITY.

Explicit description of an individual's racial or ethnic identity (e.g., "My ethnicity is Hispanic", "I identify as Mestiza", "My race is African American", "My racial identity is White", etc.).

\section{RELIGION.}

Relating to or reflecting either religious values or an individual's religious identity (e.g., "I am a good Christian woman", "Religion has had a big impact on my life", etc.)

\section{SOCIAL LIFE.}

Relating to or reflecting an individual's experiences in their social life, regardless of the presence or absence of a romantic partner.

\section{STEREOTYPING.}

Explicitly relating or referring to experiences of being stereotyped or of stereotyping others.

\section{Intersectionality: Minority Stress x Identity x Gender}

\section{AfFect.}

Relating to an individual's expressed affect (e.g., happy, sad, frustrated, angry, etc.)

\section{Appearance.}

Relating to an individual's physical appearance (e.g., body type, wearing make-up, etc.)

\section{CHILdHOOD.}

Childhood-specific experiences or behaviors

\section{CHOICE.}

Relating to an individual's freedom (or lack thereof if reverse-coded) in choosing her self-definition, roles, or behaviors. Items relevant to or reflecting choice are those that reflect some sense of autonomy for the subject of the item.

\section{COMFORT.}

Seeking or experiencing comfort. This can refer to experiencing (or seeking) comfort in general or specifically in relation to one's self-expression (e.g., an individual might describe their gender expression as being comfortable).

\section{Coming OUT.}

Coming out refers to the act of openly identifying a previously consealed facet of one's identity. Items relevant to coming out are those that either describe coming out experiences or refer to an individual's coming out experiences as part of or the entire context surrounding other experiences. 
Context Dependent.

Reflecting behaviors or experiences that are specific to or dependent upon a given context (e.g., I feel safe in LGBTQ spaces)

\section{FAMILY.}

Relating to experiences or behaviors specific to one's current or family or potential family (e.g., descriptions of individual's experiences with her parents, experiences with siblings, the possibility of marriage or having children, etc.).

\section{GENDER IDENTITY.}

Relating to or reflecting an individual's self-defined gender identity.

\section{Gender Role AdHerence.}

Relating to or reflecting adherence to a particular set of gender roles.

\section{IDENTITY FIT.}

Relating to or reflecting the fit between an individual's self-defined identity and the socially constructed roles ascribed to that identity.

\section{INTERSECTIONALITY-X.}

(Short for Intersectional Marginalization): Relating to or reflecting experiences of marginalization by others with whom an individual shares one or more marginalized identities (e.g. being ostracized by lesbian women for being bisexual racism within the LGBTQ community etc.).

\section{Media.}

Relating to or reflecting the influence of media on an individual's experiences, behaviors, or identity definitions.

\section{OTHERS' EXPECTATIONS.}

Relating to or reflecting others' expectations of an individual.

\section{Performance.}

Relating to or reflecting an individual's attempts to perform a particular role or set of roles in terms of the individual appearance, expressions, and/or behaviors.

\section{Place.}

Relating to or reflecting the concept of place as a physical environment with an identifiable and relatively static or consistent location (e.g., "I feel safe at my school"), or references to specific places such as cities, community centers, hospitals, schools, etc. (e.g., "I used to live in Los Angeles, CA", "I go to Portland State University", I enjoying going to the Q-Center, etc.).

\section{Sexual Orientation.}

Relating to or reflecting the influence of sexual orientation as a form of social categorization or as a personal identity characteristic on an individual's overall identity or specific components of a person's identity, such as their, gender, racial, ethnic, religious, and/or political identities, etc. (e.g., "I feel most comfortable hanging out with other women", "I feel like I am one of the guys", "I do not really 
think about my gender or others' genders", etc.). Items reflecting this code can also refer simply to the general concept of sexual orientation as a form of and social categorization or as a personal identity characteristic.

\section{WORK.}

Relating to or reflecting an individual's profession, experiences in the workplace, career, work-specific goals, etc. 